\title{
The Total Synthesis of Isomalabaricane Triterpenoids
}

\author{
Yaroslav D. Boyko ${ }^{\ddagger}$, Christopher J. Huck ${ }^{\ddagger}$, David Sarlah* \\ Roger Adams Laboratory, Department of Chemistry, University of Illinois, \\ Urbana, Illinois 61801, United States \\ correspondence to: David Sarlah,sarlah@illinois.edu
}

Supporting Information 


\section{TABLE OF CONTENTS}

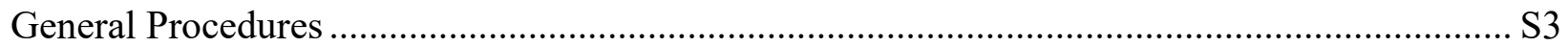

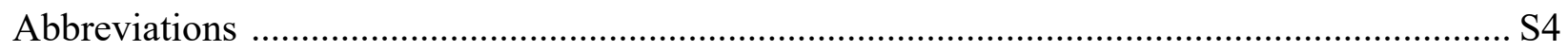

Experimental Procedures and Characterization Data............................................................. S4

Sequence for the Synthesis of Rhabdastrellic Acid A and Stelletin E.............................. S4

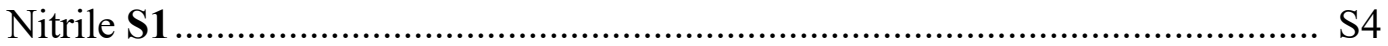

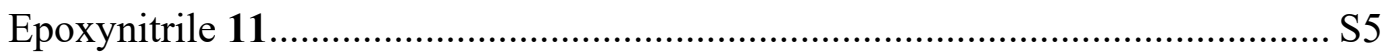

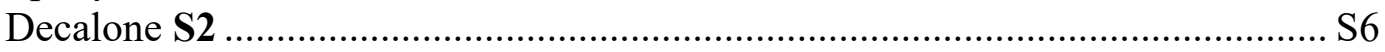

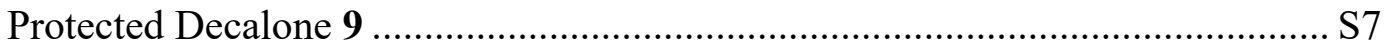

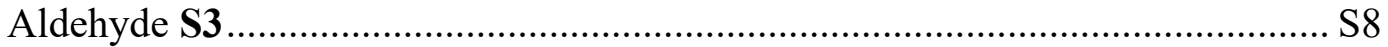

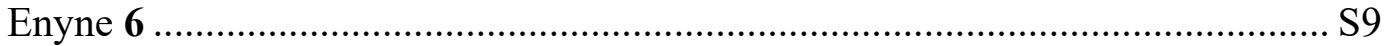

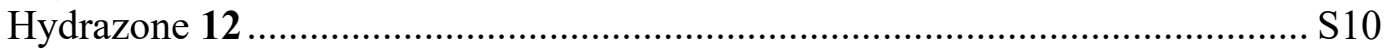

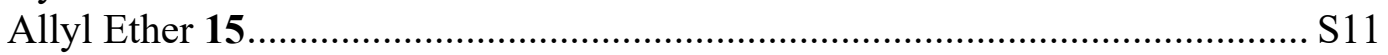

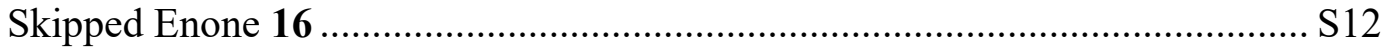

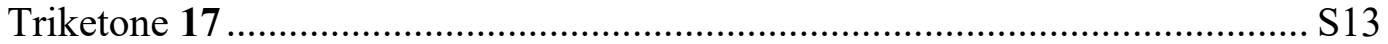

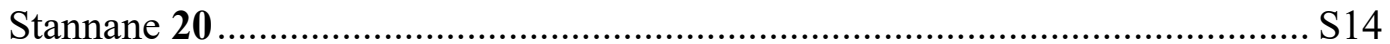

Rhabdastrellic Acid A Methyl Ester S5 .......................................................... S15

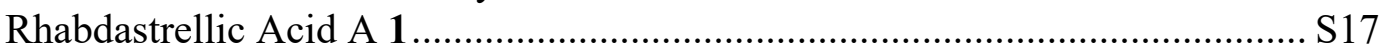

Table S1: ${ }^{13}$ C NMR Data Comparison of Synthetic Rhabdastrellic Acid A 1 ... S18

Table S2: ${ }^{1} \mathrm{H}$ NMR Data Comparison of Synthetic Rhabdastrellic Acid A 1 .... S19

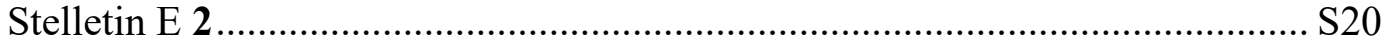

Table S3: ${ }^{13}$ C NMR Data Comparison of Synthetic Stelletin E 2 ……............... S21

Table S4: ${ }^{1} \mathrm{H}$ NMR Data Comparison of Synthetic Stelletin E 2 ………............ S22

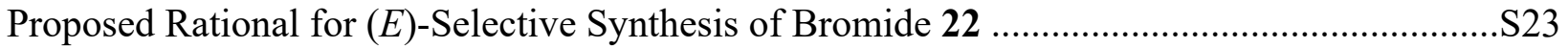

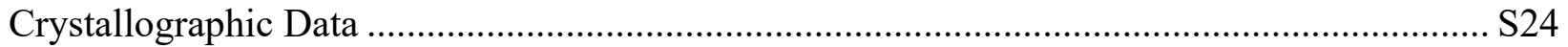

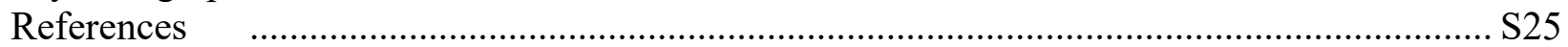

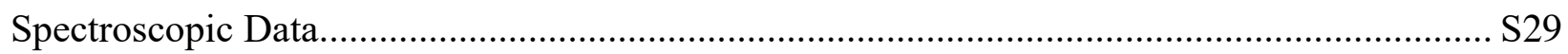




\section{General Procedures}

All chemicals were purchased from commercial suppliers and used as received, unless otherwise noted. $N$-bromosuccinimide and $\mathrm{Pd}_{2}(\mathrm{dba})_{3}{ }^{1}$ were recrystallized prior to use. C18-derivatized $\mathrm{SiO}_{2}$ was prepared according to the literature procedure. ${ }^{2}$ Diethyl ether (ACS grade), dichloromethane (ACS grade), tetrahydrofuran (HPLC grade), and toluene (ACS grade) were dried for reactions using the MB-SPS solvent purification system containing activated alumina manufactured by MBRAUN. Reaction temperatures correspond to the external temperature of the reaction vessel unless otherwise noted. Analytical thin-layer chromatography (TLC) was performed on Merck silica gel 60 F254 aluminum sheets. Visualization was accomplished with UV light and/or potassium permanganate $\left(\mathrm{KMnO}_{4}\right)$. Retention factor $\left(\mathrm{R}_{\mathrm{f}}\right)$ values reported were measured using a $10 \times 2 \mathrm{~cm}$ TLC plate in a developing chamber containing the solvent system described. Silicycle SiliaFlash ${ }^{\circledR} \mathrm{P} 60\left(\mathrm{SiO}_{2}, 40-63 \mu \mathrm{m}\right.$ particle size, $\left.230-400 \mathrm{mesh}\right)$ and aluminum oxide activated, basic, Brockmann $\mathrm{I}\left(\mathrm{Al}_{2} \mathrm{O}_{3}, 58 \AA\right.$ pore size) were used for flash column chromatography. Some compounds were purified using Biotage ${ }^{\circledR}$ Isolera ${ }^{\mathrm{TM}}$ One (AQ C18 column Spherical; $20-35 \mu \mathrm{m}$; 100A; 20g) or Shimadzu Prominence reverse phase preparative HPLC with SPD-20A UV/Vis Photodiode array detector. ${ }^{1} \mathrm{H}$ NMR spectra were obtained at $500 \mathrm{MHz},{ }^{13} \mathrm{C}$ NMR were obtained at $126 \mathrm{MHz},{ }^{19} \mathrm{~F}$ NMR were obtained at $470 \mathrm{MHz}$. NMR spectra were recorded using a Bruker Avance III $500 \mathrm{MHz}$ spectrometer equipped with BB CryoProbe or Varian/Agilent VNMRS $750 \mathrm{MHz}$ Narrow Bore and were referenced to residual chloroform $\left(7.26 \mathrm{ppm},{ }^{1} \mathrm{H}\right)$, residual benzene $\left(7.16,{ }^{1} \mathrm{H}\right)$, solvent chloroform- $d\left(77.16,{ }^{13} \mathrm{C}\right)$, solvent benzene- $d_{6}\left(128.06,{ }^{13} \mathrm{C}\right)$ or $\mathrm{PhCF}_{3}$ $\left(-62.61 \mathrm{ppm},{ }^{19} \mathrm{~F}\right)^{3}$. Chemical shifts are reported in parts per million (ppm) and multiplicities are indicated as: $\mathrm{s}$ (singlet), $\mathrm{d}$ (doublet), $\mathrm{t}$ (triplet), $\mathrm{q}$ (quartet), $\mathrm{p}$ (pentet), $\mathrm{m}$ (multiplet), and br (broad). Coupling constants, $J$, are reported in Hertz. Mass spectrometry (MS) was performed by the University of Illinois Mass Spectrometry Laboratory. Electron Impact (EI+) spectra were performed at $70 \mathrm{eV}$ using methane as the carrier gas, with time-of-flight (TOF) mass analyzer. Electrospray ionization (ES+) spectra were performed using a time-of-flight (TOF) mass analyzer. Data are reported in the form of $\mathrm{m} / \mathrm{z}$. Infrared (IR) spectra were measured neat on a Perkin-Elmer Spectrum Two FT-IR ATR spectrometer. Peaks are reported in $\mathrm{cm}^{-1}$ with indicated relative intensities: s (strong, $0-33 \% \mathrm{~T}$ ); $\mathrm{m}$ (medium, $34-66 \% \mathrm{~T}$ ), w (weak, $67-100 \% \mathrm{~T}$ ), and br (broad). Melting points were measured on a Buchi B-540 melting point apparatus and are uncorrected. Quanas 20W UV LED Light with 395-405 nm range was used for photoisomerization reaction. 
Abbreviations. $\quad$ THF $\quad=\quad$ tetrahydrofuran, $\quad \mathrm{DCE}=1,2$-Dichloroethane, NBS $=N$-Bromosuccinimide, TosMIC $=p$-Toluenesulfonylmethyl isocyanide, LDA $=$ Lithium diisopropylamide, NMP = N-Methyl-2-pyrrolidone, HMPA = Hexamethylphosphoramide, LHMDS = Lithium bis(trimethylsilyl)amide, $\mathrm{Pd}_{2}(\mathrm{dba})_{3}=$ Tris(dibenzylideneacetone)dipalladium(0), DMPU $=N, N^{\prime}$-Dimethylpropyleneurea, $\mathrm{DMF}=N, N$-Dimethylformamide, $\mathrm{IBX}=2$-Iodoxybenzoic acid, $\quad \mathrm{PCC}=$ Pyridinium chlorochromate, $\mathrm{CatBH}=$ catecholborane

\section{Experimental Procedures and Characterization Data}

\section{Sequence for the Synthesis of Isomalabaricanes}

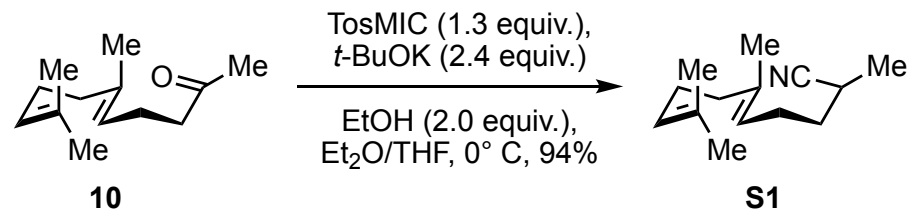

\section{(E)-2,6,10-trimethylundeca-5,9-dienenitrile (Nitrile S1):}

Geranylacetone 10 (52.4 g, 269.7 mmol, 1.0 equiv.) was dissolved in a mixture of $\mathrm{Et}_{2} \mathrm{O} / \mathrm{THF}(2.80$ L, 13:1 v:v, $0.1 \mathrm{M})$. TosMIC (68.46 g, $350.6 \mathrm{mmol}, 1.3$ equiv.) was added in one portion under inert atmosphere and the resulting white suspension was cooled to $0{ }^{\circ} \mathrm{C}$ (internal temperature). Potassium tert-butoxide (72.6 g, $647.3 \mathrm{mmol}, 2.4$ equiv.) was added in one portion as a solid. The mixture turned orange and all solids dissolved within 2 minutes, but precipitated out again in 2 minutes. The resulting suspension was treated with $\mathrm{EtOH}(31.0 \mathrm{~mL}, 539.4 \mathrm{mmol}, 2.0$ equiv. $)$ and the ice bath was removed. After 4 hours, the reaction was quenched with water $(500 \mathrm{~mL})$, the organic layer was separated, and the aqueous portion was washed with $\mathrm{Et}_{2} \mathrm{O}(3 \times 300 \mathrm{~mL})$. The combined organic extracts were washed with brine $(1000 \mathrm{~mL})$, dried over $\mathrm{MgSO}_{4}$, and concentrated at $20{ }^{\circ} \mathrm{C}$ at 120 mbar. The resulting crude oil was run through a short plug $\left(\mathrm{SiO}_{2}\right.$, $60 \% \mathrm{Et}_{2} \mathrm{O}$ in hexanes), concentrated, and dried in vacuo. Clean nitrile S1 was obtained (52.0 g, $250 \mathrm{mmol}, 94 \%$ ) as a colorless oil that was used for the next step without additional purification. A sample of high purity could be obtained by flash column chromatography $\left(\mathrm{SiO}_{2}, 25: 1\right.$, hexanes/Et $\left.{ }_{2} \mathrm{O}\right)$ or by vacuum distillation $\left(300 \mathrm{mTorr}, 115^{\circ} \mathrm{C}\right)$.

$\mathbf{R}_{\mathbf{f}} \quad 0.25\left(\mathrm{SiO}_{2}\right.$, hexanes : EtOAc $\left.=25: 1\right)$ 
${ }^{1}$ H NMR $\quad\left(500 \mathrm{MHz}, \mathrm{CDCl}_{3}\right): \delta 5.10-5.04(\mathrm{~m}, 2 \mathrm{H}), 2.61(\mathrm{dtd}, J=7.2,6.8,6.2 \mathrm{~Hz}, 1 \mathrm{H}), 2.18$ (td, $J=7.7,7.2 \mathrm{~Hz}, 2 \mathrm{H}), 2.08(\mathrm{~m}, 2 \mathrm{H}), 2.00(\mathrm{~m}, 2 \mathrm{H}), 1.70$ (dtd, $J=16.0,7.2,6.2$ $\mathrm{Hz}, 1 \mathrm{H}), 1.68(\mathrm{~s}, 3 \mathrm{H}), 1.63(\mathrm{~s}, 3 \mathrm{H}), 1.60(\mathrm{~s}, 3 \mathrm{H}), 1.55(\mathrm{dtd}, J=16.0,7.7,6.0,1 \mathrm{H})$, $1.31(\mathrm{~d}, J=6.8 \mathrm{~Hz}, 3 \mathrm{H})$

${ }^{13}$ C NMR $\quad\left(126 \mathrm{MHz}, \mathrm{CDCl}_{3}\right): \delta 137.4,131.7,124.3,123.3,122.2,39.8,34.3,26.7,25.8,25.5$, $24.9,18.1,17.8,16.2$

HRMS $\quad(\mathrm{EI}+, m / z)[\mathrm{M}]^{+}$calcd. for $\mathrm{C}_{14} \mathrm{H}_{23} \mathrm{~N}, 205.1831$; found, 205.1840 .

IR $\quad\left(A T R\right.$, neat, $\left.\mathrm{cm}^{-1}\right): 2974(\mathrm{~m}), 2923(\mathrm{~s}), 2239(\mathrm{w}), 1733(\mathrm{~s}), 1379(\mathrm{~s})$

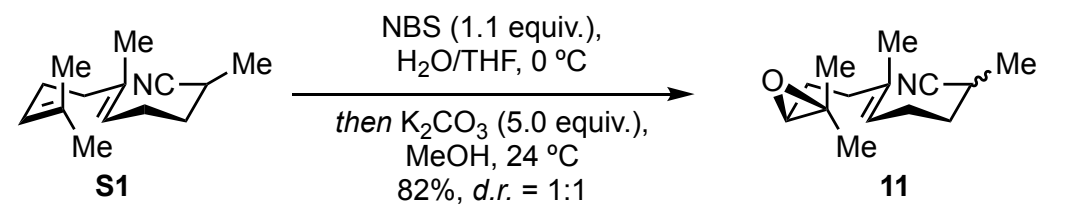

rel-(E)-8-((S)-3,3-dimethyloxiran-2-yl)-2,6-dimethyloct-5-enenitrile (Epoxynitrile 11):

To a cooled solution $\left(0^{\circ} \mathrm{C}\right.$, internal temperature) of the nitrile $\mathbf{S 1}(30.00 \mathrm{~g}, 146.1 \mathrm{mmol}, 1.0$ equiv.) in THF/ $\mathrm{H}_{2} \mathrm{O}(10.1 \mathrm{~L}, 2: 1 \mathrm{v}: \mathrm{v}, 0.0145 \mathrm{M})$ was added NBS (28.6 g, $160.7 \mathrm{mmol}, 1.1$ equiv.) as a solid in 12 portions over a period of 2 hours. The reaction mixture was stirred at this temperature for an additional 1 hour and then a solution $\mathrm{Na}_{2} \mathrm{~S}_{2} \mathrm{O}_{3}$ (sat. aq. $200 \mathrm{~mL}$ ) was added. $\mathrm{K}_{2} \mathrm{CO}_{3}(100.9 \mathrm{~g}$, $730.5 \mathrm{mmol}, 5.0$ equiv.) and $\mathrm{MeOH}(1000 \mathrm{~mL})$ were added and the resulting mixture was warmed to room temperature and stirred overnight (about six hours). The reaction volume was reduced by removing methanol and THF at $20{ }^{\circ} \mathrm{C}$ under reduced pressure. The residue was extracted with hexane $/ \mathrm{Et}_{2} \mathrm{O}(1: 1 \mathrm{v}: \mathrm{v}, 3 \times 1000 \mathrm{~mL})$. The combined organic layers were washed with brine $(1000 \mathrm{~mL})$, dried over $\mathrm{MgSO}_{4}$ and concentrated in vacuo. The crude material was passed through a short plug $\left(\mathrm{SiO}_{2}, 3: 1\right.$ hexane/Et $\left.2 \mathrm{O}\right)$ and concentrated. Clean epoxynitrile 11 was obtained $(26.30 \mathrm{~g}, 118.8 \mathrm{mmol}, 82 \%$, d.r. $=1: 1)$ as a colorless oil that was could be used in the next step without further purification. A sample of high purity could be obtained by flash column chromatography $\left(\mathrm{SiO}_{2}, 5: 1 \rightarrow 4: 1\right.$ hexanes/Et $\left.2 \mathrm{O}\right)$ or vacuum distillation $\left(300 \mathrm{mTorr}, 130^{\circ} \mathrm{C}\right)$.

$\mathbf{R}_{\mathbf{f}} \quad 0.18\left(\mathrm{SiO}_{2}\right.$, hexanes : EtOAc $\left.=10: 1\right)$

${ }^{1}$ H NMR $\quad\left(500 \mathrm{MHz}, \mathrm{CDCl}_{3}\right): \delta 5.13(\mathrm{t}, J=7.0 \mathrm{~Hz}, 1 \mathrm{H}), 2.69(\mathrm{t}, J=6.2 \mathrm{~Hz}, 1 \mathrm{H}), 2.61(\mathrm{dqd}$, $J=9.2,7.1,5.6 \mathrm{~Hz}, 1 \mathrm{H}), 2.22-2.07(\mathrm{~m}, 4 \mathrm{H}), 1.73-1.53(\mathrm{~m}, 4 \mathrm{H}), 1.66(\mathrm{~s}, 3 \mathrm{H})$, $1.31(\mathrm{~d}, J=7.1,3 \mathrm{H}), 1.30(\mathrm{~s}, 3 \mathrm{H}), 1.26(\mathrm{~s}, 3 \mathrm{H})$

${ }^{13}$ C NMR Since product consists of two diastereomers in ratio 1:1 and peaks could not be ascribed to any particular isomer, all peaks are listed. 
$\left(126 \mathrm{MHz}, \mathrm{CDCl}_{3}\right): \delta 136.5,123.2,122.83,122.81,64.22,64.21,58.4,36.54$, $36.52,34.27,34.23,27.54,27.51,25.59,25.55,25.09,25.06,25.04,18.9,18.17$, $18.15,16.2$

HRMS $\quad(\mathrm{ES}+, m / z)[\mathrm{M}+\mathrm{H}]^{+}$calcd. for $\mathrm{C}_{14} \mathrm{H}_{24} \mathrm{NO}, 222.1858$; found, 222.1863

IR

(ATR, neat, $\mathrm{cm}^{-1}$ ): 2925 (s), 2239 (w), 1667 (w), 1455 (s), 1378 (s), 1250 (m), 899 (w), $796(\mathrm{w})$

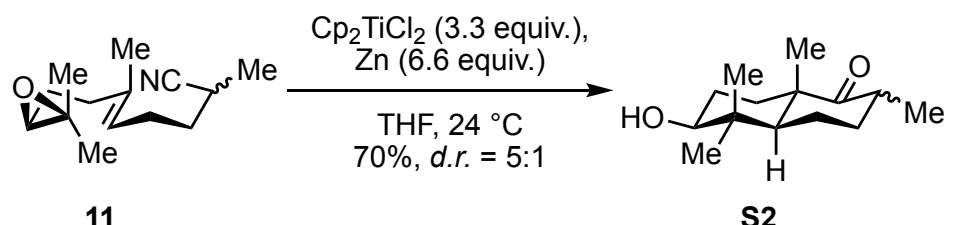

11

S2

rel-(2R,4aS,6S,8aS)-6-hydroxy-2,5,5,8a-tetramethyloctahydro-naphthalen-1(2H)-one (Decalone S2):

In an oven-dried $5 \mathrm{~L}$ round-bottom flask, titanocene dichloride (37.9 g, $152 \mathrm{mmol}, 3.3 \mathrm{equiv}$ ) and activated zinc (19.9 g, 304 mmol, 6.6 equiv.) were vigorously stirred in degassed THF (1.0 L) under nitrogen for 20 minutes. The appearance of a dark-green color indicates the formation of $\mathrm{Cp}_{2} \mathrm{TiCl}$, which was transferred dropwise to a solution of epoxynitrile 11 (10.2 g, $\left.46.1 \mathrm{mmol}\right)$ in degassed THF (3.5 L) over the course of 8 hours via cannula under an atmosphere of nitrogen. Alternatively, a dropping funnel under nitrogen can be used for this process (see Picture 1), but the slow, dropwise rate of addition is critical. Once the addition is done, the reaction was quenched with $\mathrm{NaH}_{2} \mathrm{PO}_{4}$ (sat. aq. 500 $\mathrm{mL}$ ) and left to stir overnight at ambient temperature. The resulting mixture was filtered, concentrated to remove THF, and partitioned between EtOAc $(2.0 \mathrm{~L})$ and water $(1.5 \mathrm{~L})$. The organic phase was separated and the aqueous portion was extracted with EtOAc $(2 \times 500 \mathrm{~mL})$. The combined organic extracts were washed with brine $(1.0 \mathrm{~L})$, dried over $\mathrm{MgSO}_{4}$, filtered and concentrated in vacuo. The residue was purified by flash chromatography $\left(\mathrm{SiO}_{2}, 12: 1 \rightarrow 10: 1\right.$ hexanes : EtOAc) to give the title compound (S2) $(7.20 \mathrm{~g}, 32.1 \mathrm{mmol}, 70 \%, d . r .=5: 1)$ as a colorless oil that solidifies upon storage. The major isomer $(\beta)$ was further purified by recrystallization from cold $\mathrm{MeOH}$ for

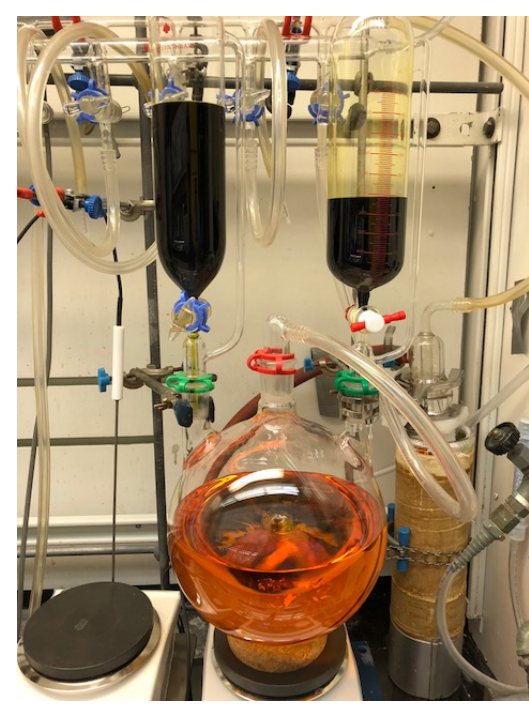

Picture 1. 10-gram scale radical cyclization. characterization.

$\mathbf{R}_{\mathbf{f}}$ $0.23\left(\mathrm{SiO}_{2}\right.$, hexanes/EtOAc $\left.=3: 1\right)$ 
T $\quad 79.7-80.9^{\circ} \mathrm{C}$

${ }^{1} \mathbf{H}$ NMR $\quad\left(500 \mathrm{MHz}, \mathrm{CDCl}_{3}\right): \delta 3.20(\mathrm{dt}, J=10.1,4.5 \mathrm{~Hz}, 1 \mathrm{H}), 2.67(\mathrm{dp}, J=12.8,6.4 \mathrm{~Hz}$, $1 \mathrm{H}), 2.12$ (ddt, $J=13.2,6.5,3.2 \mathrm{~Hz}, 1 \mathrm{H}), 1.80-1.73(\mathrm{~m}, 3 \mathrm{H}), 1.69$ (dd, $J=14.2$, $3.7 \mathrm{~Hz}, 1 \mathrm{H}), 1.62-1.57(\mathrm{~m}, 2 \mathrm{H}), 1.35(\mathrm{~d}, J=5.3 \mathrm{~Hz}, 1 \mathrm{H}), 1.21$ (ddt, $J=15.1,8.3$, $5.3 \mathrm{~Hz}, 1 \mathrm{H}), 1.14(\mathrm{~s}, 3 \mathrm{H}), 1.10(\mathrm{dd}, J=8.5,6.9 \mathrm{~Hz}, 1 \mathrm{H}), 1.00(\mathrm{~s}, 3 \mathrm{H}), 0.98$ (d, $J=6.4 \mathrm{~Hz}, 3 \mathrm{H}), 0.90(\mathrm{~s}, 3 \mathrm{H})$

${ }^{13} \mathrm{C} \mathrm{NMR} \quad\left(126 \mathrm{MHz}, \mathrm{CDCl}_{3}\right): \delta 216.2,78.3,53.4,48.5,40.0,39.9,35.7,31.5,28.1,27.2$, $21.2,19.0,15.9,15.0$

HRMS $\quad(\mathrm{EI}+, m / z)[\mathrm{M}]^{+}$calcd. for $\mathrm{C}_{14} \mathrm{H}_{24} \mathrm{O}_{2}, 224.1776$; found, 224.1780.

IR $\quad\left(\mathrm{ATR}\right.$, neat, $\left.\mathrm{cm}^{-1}\right): 3452(\mathrm{br}), 2968(\mathrm{~s}), 2933(\mathrm{~s}), 1702(\mathrm{~s}), 1141(\mathrm{~m})$

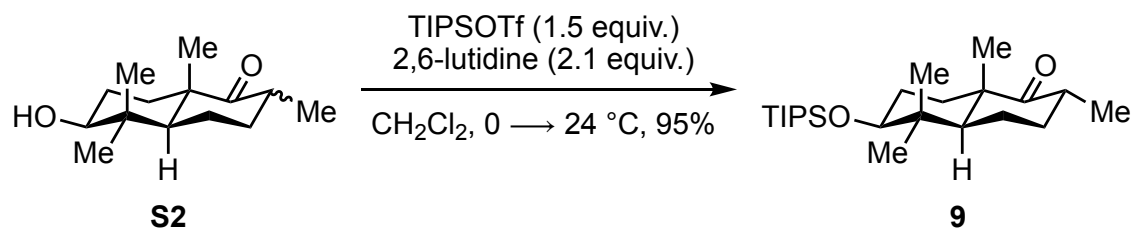

rel-(2R,4aS,6S,8aS)-2,5,5,8a-tetramethyl-6-

((triisopropylsilyl)oxy)-octahydronaphthalen-1(2H)-one (Protected Decalone 9):

To a stirred solution of decalone $\mathbf{S 2}$ (9.96 g, $44.4 \mathrm{mmol}, 1.0$ equiv.) in dry $\mathrm{CH}_{2} \mathrm{Cl}_{2}(190 \mathrm{~mL}, 0.2 \mathrm{M})$ at $0{ }^{\circ} \mathrm{C}$ under a nitrogen atmosphere, 2,6-lutidine (10.9 mL, $93.2 \mathrm{mmol}, 2.1$ equiv.) was added followed by triisopropylsilyl trifluoromethanesulfonate $(18.1 \mathrm{~mL}, 66.6 \mathrm{mmol}, 1.5$ equiv.). The reaction was warmed up to room temperature and stirred for 6 hours. Upon completion (TLC monitoring), the reaction was quenched with $\mathrm{NH}_{4} \mathrm{Cl}$ (sat. aq. $200 \mathrm{~mL}$ ). The organic phase was separated, and the aqueous layer was washed with $\mathrm{CH}_{2} \mathrm{Cl}_{2}(2 \times 100 \mathrm{~mL})$. The combined organic extracts were dried over $\mathrm{MgSO}_{4}$, filtered, and concentrated. Flash chromatography $\left(\mathrm{SiO}_{2}, 1 \%\right.$ of $\mathrm{Et}_{2} \mathrm{O}$ in hexanes) provided the desired compound 9 as a white solid (16.0 g, $\left.42.0 \mathrm{mmol}, 95 \%\right)$

$\mathbf{R}_{\mathbf{f}} \quad 0.15\left(\mathrm{SiO}_{2}\right.$, hexanes : EtOAc $\left.=99: 1\right)$

T melt. $\quad 98.9^{\circ} \mathrm{C}$

${ }^{1} \mathbf{H}$ NMR $\quad\left(500 \mathrm{MHz}, \mathrm{CDCl}_{3}\right): \delta 3.37-3.34(\mathrm{~m}, 1 \mathrm{H}), 2.67(\mathrm{dp}, J=12.8,6.4 \mathrm{~Hz}, 1 \mathrm{H}), 2.10$ (ddt, $J=13.0,6.4,3.3 \mathrm{~Hz}, 1 \mathrm{H}), 1.79-1.70(\mathrm{~m}, 3 \mathrm{H}), 1.65-1.58(\mathrm{~m}, 2 \mathrm{H}), 1.55(\mathrm{~m}$, $1 \mathrm{H}), 1.23-1.15(\mathrm{~m}, 1 \mathrm{H}), 1.14(\mathrm{~s}, 3 \mathrm{H}), 1.10-1.07(\mathrm{~m}, 1 \mathrm{H}), 1.06(\mathrm{~s}, 21 \mathrm{H}), 0.99(\mathrm{~s}$, $3 \mathrm{H}), 0.97(\mathrm{~d}, J=6.4 \mathrm{~Hz}, 3 \mathrm{H}), 0.89(\mathrm{~s}, 3 \mathrm{H})$ 
${ }^{13} \mathbf{C}$ NMR $\quad\left(126 \mathrm{MHz}, \mathrm{CDCl}_{3}\right): \delta 216.6,79.4,53.7,48.5,41.0,40.0,35.9,31.5,28.5,27.5$, $21.4,19.0,18.5,18.4,16.3,15.1,13.2$

HRMS $\quad(\mathrm{CI}+, m / z)[\mathrm{M}+\mathrm{H}]^{+}$calcd. for $\mathrm{C}_{23} \mathrm{H}_{45} \mathrm{O}_{2} \mathrm{Si}$, 381.3189; found, 381.3175 .

IR (ATR, neat, $\left.\mathrm{cm}^{-1}\right): 2942(\mathrm{~m}), 2865$ (s), 1699 (s), 1457 (w), $1115(\mathrm{~s}), 881(\mathrm{~m}), 667$

(s)

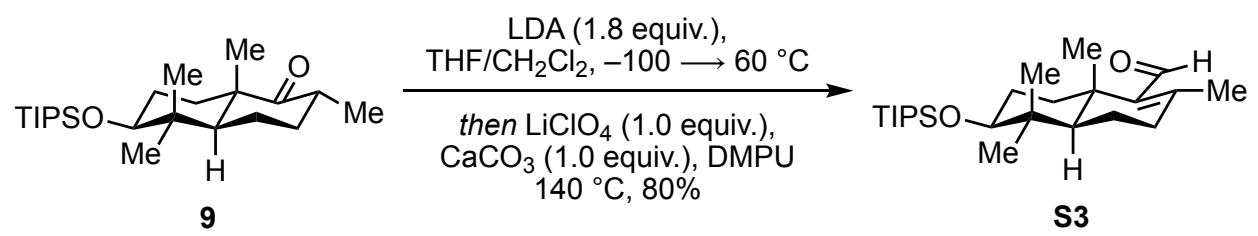

rel-(4a $R, 6 S, 8 \mathrm{a} S)-2,5,5,8 \mathrm{a}-$ tetramethyl-6-((triisopropylsilyl)oxy)-3,4,4a,5,6,7,8,8aoctahydronaphthalene-1-carbaldehyde (Aldehyde S3):

Caution! Product isomerizes on $\mathrm{SiO}_{2}$ to the $\beta, \gamma$-unsaturated aldehyde. In an oven-dried roundbottom flask, a solution of LDA ( $7.0 \mathrm{~mL}, 0.7 \mathrm{M}$ in THF, $4.68 \mathrm{mmol}, 1.8$ equiv.) was prepared in situ and cooled down to $-100{ }^{\circ} \mathrm{C}$ under nitrogen atmosphere. After 20 minutes, a solution of decalone 9 (1.00 g, $2.63 \mathrm{mmol}, 1.0$ equiv.) in $\mathrm{CH}_{2} \mathrm{Cl}_{2}(2.6 \mathrm{~mL})$ was added dropwise, over the course of 10 minutes, with careful monitoring of the low temperature in an EtOH/liq. $\mathrm{N}_{2}$ bath. The mixture was allowed to warm up to $-20{ }^{\circ} \mathrm{C}$ over 2 hours and then followed by a gentle reflux at $60^{\circ} \mathrm{C}$ for an additional 1 hour. The resulting black solution was cooled down to room temperature, concentrated under reduced pressure, and redissolved in DMPU $(4.5 \mathrm{~mL}) . \mathrm{LiClO}_{4}(280 \mathrm{mg}, 2.63$ mmol, 1.0 equiv.) and $\mathrm{CaCO}_{3}(263 \mathrm{mg}, 2.63 \mathrm{mmol}, 1.0$ equiv.) were added sequentially with vigorous stirring, and the suspension was heated up to $140{ }^{\circ} \mathrm{C}$ for 1.5 hours. Finally, the reaction was cooled down to room temperature, carefully quenched with aqueous $\mathrm{HCl}(3 \mathrm{M}, 5 \mathrm{~mL})$, and partitioned between $\mathrm{Et}_{2} \mathrm{O}(30 \mathrm{~mL})$ and water $(30 \mathrm{~mL})$. The organic phase was separated and the aqueous layer was washed with $\mathrm{Et}_{2} \mathrm{O}(3 \times 20 \mathrm{~mL})$. The combined organic fractions were washed with brine $(30 \mathrm{~mL})$, dried over $\mathrm{MgSO}_{4}$, filtered, and concentrated. Flash chromatography (basic $\mathrm{Al}_{2} \mathrm{O}_{3}, 0 \% \rightarrow 5 \% \mathrm{Et}_{2} \mathrm{O}$ in hexanes) furnished $\mathbf{S 3}(820 \mathrm{mg}, 2.63 \mathrm{mmol}, 80 \%)$.

$\mathbf{R}_{\mathbf{f}} \quad 0.30\left(\mathrm{SiO}_{2}\right.$, hexanes : $\left.\mathrm{EtOAc}=25: 1\right)$

T T $_{\text {melt. }} \quad 75.7-76.8^{\circ} \mathrm{C}$

${ }^{1}$ H NMR $\quad\left(500 \mathrm{MHz}, \mathrm{CDCl}_{3}\right) \delta 10.03(\mathrm{~s}, 1 \mathrm{H}), 3.41(\mathrm{dd}, J=11.0,5.1 \mathrm{~Hz}, 1 \mathrm{H}), 2.59(\mathrm{dt}, 13.4$, $3.6 \mathrm{~Hz}, 1 \mathrm{H}), 2.28$ (m, 2H), 2.03 (s, 3H), $1.75-1.64$ (m, 3H), 1.50 (tdd, $J=13.1$, $10.1,7.2 \mathrm{~Hz}, 1 \mathrm{H}), 1.17(\mathrm{~s}, 3 \mathrm{H}), 1.09-1.03(\mathrm{~m}, 23 \mathrm{H}), 1.02(\mathrm{~s}, 3 \mathrm{H}), 0.82(\mathrm{~s}, 3 \mathrm{H})$ 
${ }^{13}$ C NMR $\quad\left(126 \mathrm{MHz}, \mathrm{CDCl}_{3}\right): \delta 192.4,154.4,143.5,79.9,51.3,40.2,37.4,37.0,34.5,28.8$, $28.3,20.2,19.1,18.52,18.45,18.41,16.2,13.2$

HRMS (ES+, $m / z)$ calcd. for $\mathrm{C}_{24} \mathrm{H}_{45} \mathrm{O}_{2} \mathrm{Si}[\mathrm{M}+\mathrm{H}]^{+}$calcd.: 393.3189 ; found: 393.3208 .

IR (ATR, neat, $\mathrm{cm}^{-1}$ ): 2939 (m), 2864 (s), 1669 (s), 1612 (w), $1111(\mathrm{~s}), 882(\mathrm{~m}), 679(\mathrm{~s})$

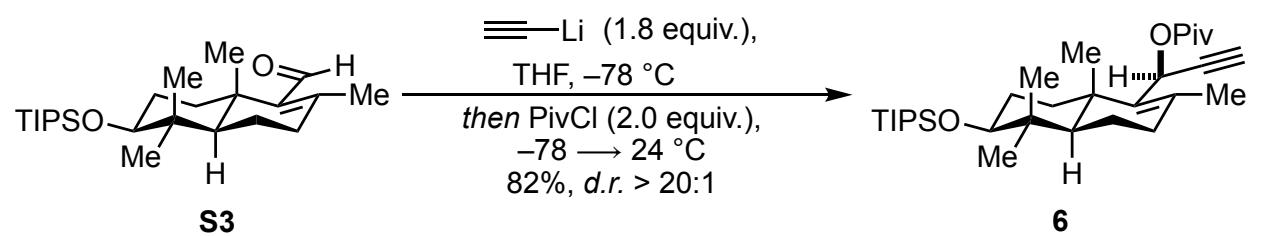

rel-(R)-1-((4aR,6S,8aS)-2,5,5,8a-tetramethyl-6-((triisopropylsilyl)oxy)-3,4,4a,5,6,7,8,8aoctahydronaphthalen-1-yl)prop-2-yn-1-yl pivalate (Enyne 6):

To a solution of freshly prepared lithium acetylide in THF ( $200 \mathrm{~mL}, 0.1 \mathrm{M}, 20.6 \mathrm{mmol}, 1.8$ equiv.) at $-78^{\circ} \mathrm{C}$, a solution of the aldehyde $\mathbf{S 3}(4.50 \mathrm{~g}, 11.5 \mathrm{mmol}, 1.0$ equiv.) in THF $(30 \mathrm{~mL})$ was added dropwise over the course of $5 \mathrm{~min}$. The reaction was maintained at $-78^{\circ} \mathrm{C}$ for $3 \mathrm{~h}$. Upon completion (TLC monitoring), pivaloyl chloride ( $2.8 \mathrm{~mL}, 22.9 \mathrm{mmol}, 2.0$ equiv.) was added and the resulting solution was slowly warmed up to ambient temperature and stirred for additional 4 hours at room temperature. The reaction was quenched with $\mathrm{NH}_{4} \mathrm{OH}$ (aq. $2 \mathrm{M}, 200 \mathrm{~mL}$ ), THF was removed under reduced pressure and resulting solution was partitioned between $\mathrm{Et}_{2} \mathrm{O}(300 \mathrm{~mL})$ and water $(100 \mathrm{~mL})$. The organic layer was separated and the aqueous phase was washed with $\mathrm{Et}_{2} \mathrm{O}$ $\left(2 \times 100 \mathrm{~mL}\right.$ ). The combined organic fractions were vigorously washed with $\mathrm{NH}_{4} \mathrm{OH}$ (aq. $2 \mathrm{M}$, $2 \times 200 \mathrm{~mL}$ ) and brine $(200 \mathrm{~mL})$, dried over $\mathrm{MgSO}_{4}$, filtered and concentrated. This crude residue was purified by flash chromatography $\left(\mathrm{C}_{18}\right.$ reverse phase $\mathrm{SiO}_{2}$, gradient $90 \% \rightarrow 100 \% \mathrm{MeCN}$ in $\mathrm{H}_{2} \mathrm{O}$ ), which afforded $\mathbf{6}$ as a white crystalline material (4.70 g, $9.35 \mathrm{mmol}, 82 \%$, d.r. $\left.>20: 1\right)$.

$\mathbf{R}_{\mathbf{f}} \quad 0.68\left(\mathrm{SiO}_{2}\right.$, hexanes : EtOAc $\left.=20: 1\right)$

T melt. $\quad 97.4-98.9^{\circ} \mathrm{C}$

${ }^{1} \mathbf{H}$ NMR $\quad\left(500 \mathrm{MHz}, \mathrm{CDCl}_{3}\right): \delta 6.01(\mathrm{~d}, J=2.4 \mathrm{~Hz}, 1 \mathrm{H}), 3.38(\mathrm{~m}, 1 \mathrm{H}), 2.44(\mathrm{~d}, J=2.4 \mathrm{~Hz}$, $1 \mathrm{H}), 2.12-2.09(\mathrm{~m}, 2 \mathrm{H}), 1.89(\mathrm{~s}, 3 \mathrm{H}), 1.83(\mathrm{dt}, J=13.0,3.6 \mathrm{~Hz}, 1 \mathrm{H}), 1.71-1.64(\mathrm{~m}$, $3 \mathrm{H}), 1.46$ (tdd, $J=13.0,10.2,7.7 \mathrm{~Hz}, 1 \mathrm{H}), 1.21(\mathrm{~s}, 9 \mathrm{H}), 1.15(\mathrm{dd}, J=11.2,5.2 \mathrm{~Hz}$, $1 \mathrm{H}), 1.08-1.04(\mathrm{~m}, 1 \mathrm{H}), 1.06(\mathrm{~s}, 21 \mathrm{H}), 1.03(\mathrm{~s}, 3 \mathrm{H}), 1.00(\mathrm{~s}, 3 \mathrm{H}), 0.79(\mathrm{~s}, 3 \mathrm{H})$ 
${ }^{13}$ C NMR $\quad\left(126 \mathrm{MHz}, \mathrm{CDCl}_{3}\right): \delta 177.3,136.7,136.4,82.5,79.8,72.7,60.1,51.4,40.0,38.9$, $38.8,35.2,34.4,28.7,28.2,27.2,21.1,20.7,18.8,18.52,18.46,16.2,13.2$

HRMS $\quad(\mathrm{EI}+, \mathrm{m} / z)[\mathrm{M}]^{+}$calcd. for $\mathrm{C}_{31} \mathrm{H}_{54} \mathrm{O}_{3} \mathrm{Si}, 502.3842$; found, 502.3855 .

IR (ATR, neat, $\left.\mathrm{cm}^{-1}\right): 3311(\mathrm{w}), 2942(\mathrm{~m}), 2866(\mathrm{~m}), 1736(\mathrm{~s}), 1141(\mathrm{~s}), 1113(\mathrm{~s}), 1067$ $(\mathrm{w}), 882(\mathrm{~m})$

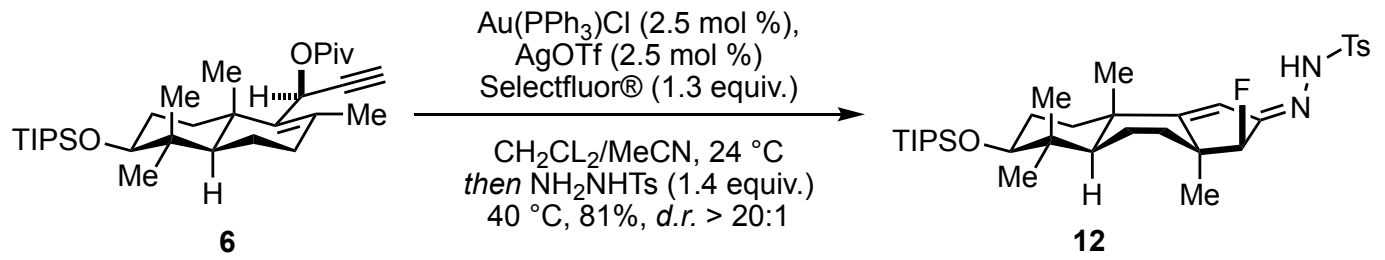

rel- $N^{\prime}$-((3S,3a $\left.S, 5 \mathrm{a} R, 7 S, 9 \mathrm{a} S\right)$-3-fluoro-3a,6,6,9a-tetramethyl-7-((triisopropylsilyl)oxy)-

3,3a,4,5,5a,6,7,8,9,9a-decahydro-2H-cyclopenta $[a]$ naphthalen-2-ylidene)-4methylbenzenesulfonohydrazide (Hydrazone 12):

Enyne 6 (920 mg, $1.83 \mathrm{mmol}, 1.0$ equiv.) was dissolved in the mixture $\mathrm{MeCN}: \mathrm{CH}_{2} \mathrm{Cl}_{2}=2.5: 1$ (9.1 mL, $0.20 \mathrm{M})$. SelectFluor ${ }^{\circledR}(843 \mathrm{mg}, 2.92 \mathrm{mmol}, 1.3$ equiv.) was added. A separate 4-mL vial was charged with [Au( $\left.\mathrm{PPh}_{3}\right) \mathrm{Cl}$ ] (23 mg, $0.046 \mathrm{mmol}, 2.5 \mathrm{~mol} \%$ ) and AgOTf $(12 \mathrm{mg}, 0.046 \mathrm{mmol}$, $2.5 \mathrm{~mol} \%)$ inside of a nitrogen-filled glovebox. Dry $\mathrm{CH}_{2} \mathrm{Cl}_{2}(0.5 \mathrm{~mL})$ was added under inert atmosphere and stirred at room temperature with protection from light. After 10 minutes, precipitated $\mathrm{AgCl}$ was visible and the suspension was transferred into the reaction mixture. Conversion was monitored by TLC. Once full conversion was achieved (about 1-2 hours), $p$-toluenesulfonyl hydrazide (511 $\mathrm{mg}, 2.74 \mathrm{mmol}, 1.4$ equiv.) was added and the reaction was heated to $40{ }^{\circ} \mathrm{C}$ for $10 \mathrm{~h}$. The resulting suspension was partitioned between $\mathrm{CH}_{2} \mathrm{Cl}_{2}(40 \mathrm{~mL})$ and water $(80 \mathrm{~mL})$. The organic layer was separated and the aqueous layer was washed with $\mathrm{CH}_{2} \mathrm{Cl}_{2}$ $(3 \times 20 \mathrm{~mL})$. The combined organic phases were dried over $\mathrm{MgSO}_{4}$, filtered, and concentrated in vacuo. Hydrazone 12 (900 mg, $1.49 \mathrm{mmol}, 81 \%$, d.r. > 20:1) was isolated as a white solid using the Biotage ${ }^{\circledR}$ Isolera $^{\mathrm{TM}}$ One (AQ C18 column Spherical; $20-35 \mu \mathrm{m} ; 100 \mathrm{~A} ; 20 \mathrm{~g}, 75 \% \rightarrow 100 \%$ $\mathrm{MeCN}$ in $\mathrm{H}_{2} \mathrm{O}$, detection at $\left.\lambda=275 \mathrm{~nm}\right)$.

$\mathbf{R}_{\mathbf{f}} \quad 0.41\left(\mathrm{SiO}_{2}\right.$, hexanes : EtOAc $\left.=5: 1\right)$

T $\quad 169.3-171.2^{\circ} \mathrm{C}$

${ }^{1}$ H NMR $\quad\left(500 \mathrm{MHz}, \mathrm{CDCl}_{3}\right): \delta 7.86(\mathrm{~d}, J=8.4 \mathrm{~Hz}, 2 \mathrm{H}), 7.66(\mathrm{~s}, 1 \mathrm{H}), 7.31(\mathrm{~d}, J=8.4 \mathrm{~Hz}$, 2H), $5.95(\mathrm{~s}, 1 \mathrm{H}), 4.65(\mathrm{~d}, J=55.7 \mathrm{~Hz}, 1 \mathrm{H}), 3.37(\mathrm{dd}, J=10.3,5.3 \mathrm{~Hz}, 1 \mathrm{H}), 2.42$ 
$(\mathrm{s}, 3 \mathrm{H}), 1.95(\mathrm{dt}, J=13.6,9.5 \mathrm{~Hz}, 1 \mathrm{H}), 1.88-1.80(\mathrm{~m}, 2 \mathrm{H}), 1.78-1.67(\mathrm{~m}, 3 \mathrm{H})$, $1.59-1.52(\mathrm{~m}, 2 \mathrm{H}), 1.36(\mathrm{~m}, 1 \mathrm{H}), 1.12(\mathrm{~d}, J=2.2 \mathrm{~Hz}, 3 \mathrm{H}), 1.09(\mathrm{~s}, 3 \mathrm{H}), 1.07(\mathrm{~s}$, $21 \mathrm{H}), 0.99$ (s, 3H), 0.88 (s, 3H)

${ }^{13}$ C NMR $\quad\left(126 \mathrm{MHz}, \mathrm{CDCl}_{3}\right): \delta 184.5(\mathrm{~d}, J=3.3 \mathrm{~Hz}), 159.5(\mathrm{~d}, J=14.6 \mathrm{~Hz}), 144.3,135.4$, $129.8,128.1,110.3,99.2$ (d, $J=189.4 \mathrm{~Hz}), 80.0,48.2$ (d, $J=19.4 \mathrm{~Hz}), 43.2,40.5$, $38.7,36.8,28.6,28.2,26.8(\mathrm{~d}, J=4.8 \mathrm{~Hz}), 25.1,22.6(\mathrm{~d}, J=9.3 \mathrm{~Hz}), 21.8,18.52$, $18.45,16.7,15.9,13.2$

${ }^{19}$ F NMR $\quad\left(470 \mathrm{MHz}, \mathrm{CDCl}_{3}\right): \delta-178.0(\mathrm{~d}, J=55.7 \mathrm{~Hz})$

HRMS $(\mathrm{ES}+, \mathrm{m} / z)[\mathrm{M}+\mathrm{H}]^{+}$calcd. for $\mathrm{C}_{33} \mathrm{H}_{54} \mathrm{~N}_{2} \mathrm{O}_{3} \mathrm{SiSF}, 605.3608$; found, 605.3587 .

IR

(ATR, neat, $\mathrm{cm}^{-1}$ ): 3216 (br), 2942 (s), 2866 (s), 1597 (w), 1462 (m), 1345 (s), $1165(\mathrm{~s})$

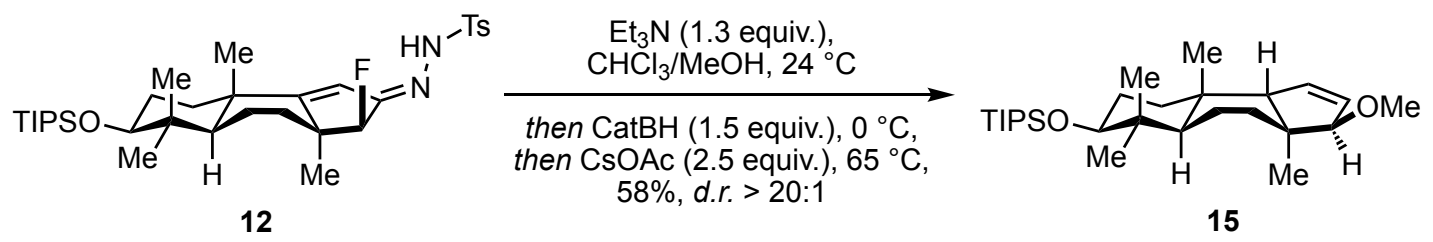

rel-triisopropyl(((3R,3aS,5a $R, 7 S, 9 \mathrm{a} S, 9 \mathrm{~b} S)-3-$ methoxy-3a,6,6,9a-tetramethyl-

3a,4,5,5a,6,7,8,9,9a,9b-decahydro-3H-cyclopenta[a]naphthalen-7-yl)oxy)silane

(Allyl

Ether 15):

The $\alpha$-fluorohydrazone 12 (960 $\mathrm{mg}, 1.56 \mathrm{mmol}, 1.0$ equiv.) was dissolved in the mixture $\mathrm{CHCl}_{3} / \mathrm{MeOH}(2: 1,16 \mathrm{~mL}, 0.1 \mathrm{M})$. The solution was treated with $\mathrm{Et}_{3} \mathrm{~N}(0.28 \mathrm{~mL}, 2.03 \mathrm{mmol}$, 1.3 equiv.) and stirred for $2 \mathrm{~h}$. The solvent and excess of $\mathrm{Et}_{3} \mathrm{~N}$ were removed in vacuo. The remaining yellow foam was redissolved in dry and degassed $\mathrm{CHCl}_{3}$ $(16 \mathrm{~mL}, 0.1 \mathrm{M})$ under a nitrogen atmosphere, cooled down to $0{ }^{\circ} \mathrm{C}$. Catecholborane (250 $\mu \mathrm{L}, 2.33 \mathrm{mmol}, 1.5$ equiv.) was added in dropwise. After $5 \mathrm{~min}$, the yellow suspension was warmed up to room temperature and stirred for additional $3 \mathrm{~h}$. CsOAc (747 mg, $3.89 \mathrm{mmol}$, 2.5 equiv.) was added in one portion as a solid and the mixture was heated to $65^{\circ} \mathrm{C}$ for another $4 \mathrm{~h}$. The thick white suspension was cooled down to ambient temperature and filtered through celite. This filtrate was concentrated and purified by flash chromatography $\left(\mathrm{SiO}_{2}, 1 \%\right.$ of $\mathrm{Et}_{2} \mathrm{O}$ in hexanes). Allyl ether 15 (395 mg, $0.909 \mathrm{mmol}, 58 \%$, d.r. $>20: 1$ ) was isolated as a clear pale-yellow oil (see Picture 2).

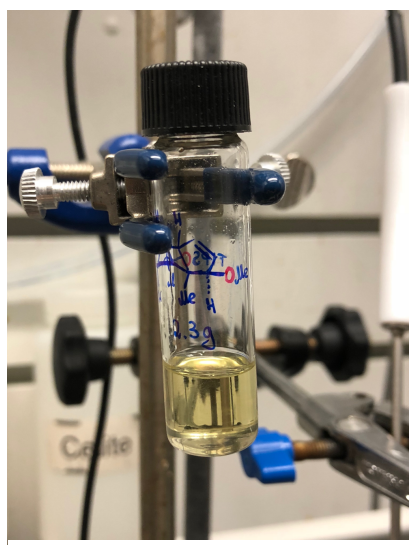

Picture 2. 2.3 grams can be made in a single pass.

$$
\mathbf{R}_{\mathbf{f}} \quad 0.42\left(\mathrm{SiO}_{2} \text {, hexanes : EtOAc }=20: 1\right)
$$


${ }^{1}$ H NMR $\quad\left(500 \mathrm{MHz}, \mathrm{CDCl}_{3}\right): \delta 6.14(\mathrm{dd}, J=6.0,1.8 \mathrm{~Hz}, 1 \mathrm{H}), 6.05(\mathrm{ddd}, J=6.0,3.6,2.4$ $\mathrm{Hz}, 1 \mathrm{H}), 3.42(\mathrm{dd}, J=10.6,5.8 \mathrm{~Hz}, 1 \mathrm{H}), 3.36(\mathrm{~d}, J=2.4 \mathrm{~Hz}, 1 \mathrm{H}), 3.29(\mathrm{~s}, 3 \mathrm{H}), 2.76$ $(\mathrm{dd}, J=3.6,1.8 \mathrm{~Hz}, 1 \mathrm{H}), 2.19(\mathrm{dd}, J=12.9,8.7 \mathrm{~Hz}, 1 \mathrm{H}), 1.76-1.70(\mathrm{~m}, 3 \mathrm{H}), 1.67$ $(\mathrm{dd}, J=13.5,9.0 \mathrm{~Hz}, 1 \mathrm{H}), 1.48(\mathrm{dt}, J=13.0,3.7 \mathrm{~Hz}, 1 \mathrm{H}), 1.42(\mathrm{dt}, J=12.3,9.0 \mathrm{~Hz}$, $1 \mathrm{H}), 1.37-1.29(\mathrm{~m}, 2 \mathrm{H}), 1.14(\mathrm{~s}, 3 \mathrm{H}), 1.07(\mathrm{~s}, 21 \mathrm{H}), 1.02(\mathrm{~s}, 3 \mathrm{H}), 1.00(\mathrm{~s}, 3 \mathrm{H})$, $0.79(\mathrm{~s}, 3 \mathrm{H})$

${ }^{13}$ C NMR $\quad\left(126 \mathrm{MHz}, \mathrm{CDCl}_{3}\right): \delta 136.9,131.5,91.2,80.8,57.7,57.5,47.1,46.5,40.4,35.1$, $33.7,29.9,29.8,28.4,27.0,23.9,18.8,18.6,18.5,16.6,13.2$

HRMS $\quad(\mathrm{ES}+, \mathrm{m} / z)\left[\mathrm{M}-\mathrm{OCH}_{3}\right]^{+}$calcd. for $\mathrm{C}_{26} \mathrm{H}_{47} \mathrm{OSi}$, 403.3396; found, 403.3403 .

IR

(ATR, neat, $\mathrm{cm}^{-1}$ ): 2940 (br), 2866 (s), 1463 (w), 1106 (m), 1057 (w)

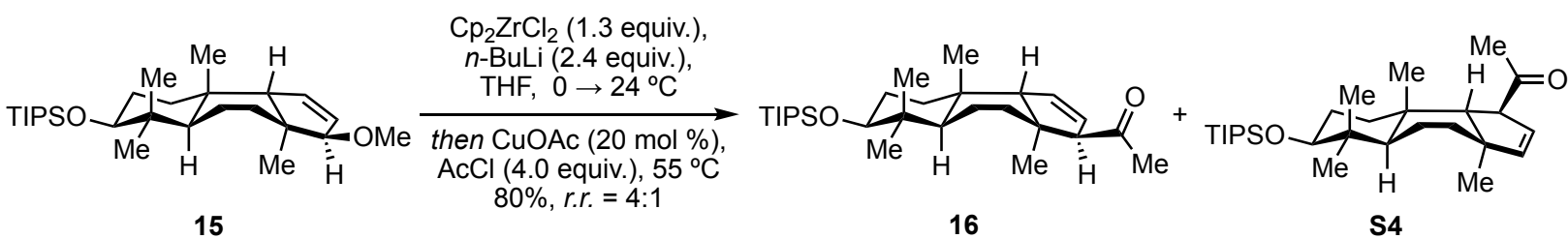

rel-1-((3R,3aS,5a $R, 7 S, 9 \mathrm{a} S, 9 \mathrm{~b} S)-3 \mathrm{a}, 6,6,9 \mathrm{a}-t$ tramethyl-7-((triisopropylsilyl)oxy)3a,4,5,5a,6,7,8,9,9a,9b-decahydro-3H-cyclopenta[ $[a]$ naphthalen-3-yl)ethan-1-one (Skipped Enone 16):

To a solution of 15 ( $80 \mathrm{mg}, 0.18 \mathrm{mmol}, 1.0$ equiv.) and $\mathrm{Cp}_{2} \mathrm{ZrCl}_{2}$ (70 mg, 0.24 mmol, 1.3 equiv.) in THF $(0.32 \mathrm{~mL}, 0.6 \mathrm{M})$ was added dropwise a solution of $n$-BuLi $(0.28 \mathrm{~mL}, 1.6 \mathrm{M}, 0.44 \mathrm{mmol}$, 2.4 equiv.) under a nitrogen atmosphere at $0{ }^{\circ} \mathrm{C}$. The yellow reaction mixture underwent a color change to brown. The ice bath was removed after 10 minutes and the mixture was stirred at ambient temperature for 36 hours. A separate 4-mL vial was charged with CuOAc (4.5 mg, $0.037 \mathrm{mmol}$, 0.20 equiv.) inside of a glovebox, and a suspension was prepared in THF $(0.9 \mathrm{~mL})$. This suspension was heated to $55^{\circ} \mathrm{C}$ and treated with acetyl chloride $(0.053 \mathrm{~mL}, 0.74 \mathrm{mmol}, 4.0$ equiv. $)$. The orange allylzirconium in the first flask was transferred via cannula to the copper catalyst and acetyl chloride in the second vessel. An additional portion of THF $(0.3 \mathrm{~mL})$ was used for washing the initial flask and then transferred to the second vessel by cannulation as well. After stirring for 3 hours at $55^{\circ} \mathrm{C}$ the reaction was quenched with $1 \mathrm{M} \mathrm{HCl}(1 \mathrm{~mL})$ and partitioned between EtOAc $(7 \mathrm{~mL})$ and water $(5 \mathrm{~mL})$. The organic layer was separated and the aqueous phase was washed with EtOAc $(2 \times 5 \mathrm{~mL})$. The combined organic fractions were washed with brine $(10 \mathrm{~mL})$, dried over $\mathrm{MgSO}_{4}$, filtered and concentrated. This material was purified by flash chromatography (1\% EtOAc in hexanes) to give skipped enone 16 (53 $\mathrm{mg}, 0.12 \mathrm{mmol}, 64 \%)$ and its constitutional isomer $\mathbf{S 4}$ (13 $\mathrm{mg}, 0.029 \mathrm{mmol}, 16 \%$ ) as crystalline solids in a ratio of 4:1.

$\mathbf{R}_{\mathbf{f}}$

$0.48\left(\mathrm{SiO}_{2}\right.$, hexanes : EtOAc $\left.=40: 1\right)$ 
T $\quad 76.5-77.3{ }^{\circ} \mathrm{C}$

${ }^{1}$ H NMR $\quad\left(500 \mathrm{MHz}, \mathrm{CDCl}_{3}\right): \delta 6.11(\mathrm{~d}, J=5.7 \mathrm{~Hz}, 1 \mathrm{H}), 5.76(\mathrm{dt}, J=6.1,3.2 \mathrm{~Hz}, 1 \mathrm{H}), 3.42$ $(\mathrm{dd}, J=9.7,6.8 \mathrm{~Hz}, 1 \mathrm{H}), 3.06(\mathrm{~d}, J=2.7 \mathrm{~Hz}, 1 \mathrm{H}), 2.77(\mathrm{~d}, J=3.6 \mathrm{~Hz}, 1 \mathrm{H}), 2.11(\mathrm{~s}$, $3 \mathrm{H}), 1.83(\mathrm{dd}, J=13.4,8.9 \mathrm{~Hz}, 1 \mathrm{H}), 1.77-1.71(\mathrm{~m}, 2 \mathrm{H}), 1.68(\mathrm{~m}, 1 \mathrm{H}), 1.64$ (dd, $J=13.1,8.9 \mathrm{~Hz}, 1 \mathrm{H}), 1.56-1.50(\mathrm{~m}, 2 \mathrm{H}), 1.44-1.35(\mathrm{~m}, 2 \mathrm{H}), 1.32(\mathrm{~s}, 3 \mathrm{H}), 1.08$ $(\mathrm{s}, 21 \mathrm{H}), 1.03(\mathrm{~s}, 3 \mathrm{H}), 0.98(\mathrm{~s}, 3 \mathrm{H}), 0.78(\mathrm{~s}, 3 \mathrm{H})$

${ }^{13} \mathbf{C ~ N M R ~} \quad\left(126 \mathrm{MHz}, \mathrm{CDCl}_{3}\right): \delta 209.6,134.6,130.6,80.7,70.3,58.7,49.3,45.7,40.4,35.6$, $33.6,32.1,31.1,30.3,29.9,29.8,24.1,18.8,18.6,18.5,16.6,13.2$

HRMS $\quad(\mathrm{ES}+, m / z)[\mathrm{M}+\mathrm{H}]^{+}$calcd. for $\mathrm{C}_{28} \mathrm{H}_{51} \mathrm{O}_{2} \mathrm{Si}$, 447.3658; found, 447.3662.

IR (ATR, neat, $\mathrm{cm}^{-1}$ ): 2942 (br), 2865 (s), 1708 (m), 1463 (w), $1111(\mathrm{~m})$

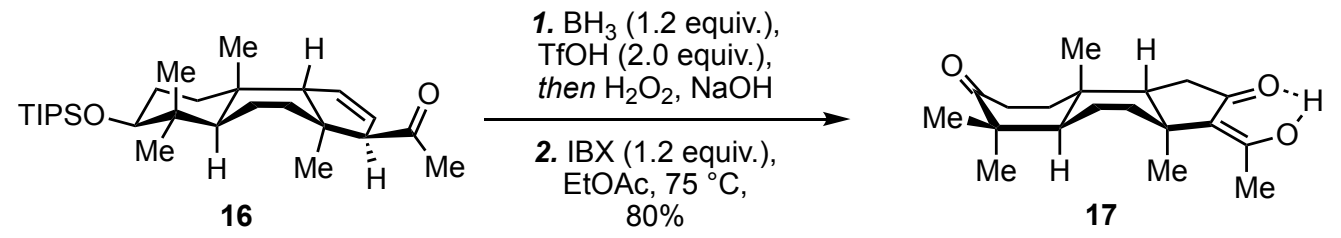

rel-(3a $S, 5 \mathrm{a} R, 9 \mathrm{a} R, 9 \mathrm{~b} S, Z)-3-(1-h y d r o x y e t h y l i d e n e)-3 \mathrm{a}, 6,6,9 \mathrm{a}-$ tetramethyldecahydro-1Hcyclopenta $[a]$ naphthalene-2,7-dione (Triketone 17):

The enone 16 (36 mg, $0.081 \mathrm{mmol}, 1.0$ equiv.) was dissolved in dry THF (0.8 mL, $0.1 \mathrm{M})$ under a nitrogen atmosphere and cooled to $-20^{\circ} \mathrm{C}$. The substrate was treated with $\mathrm{BH}_{3} \bullet \mathrm{Me}_{2} \mathrm{~S}(9.2 \mu \mathrm{l}, 0.097$ mmol, 1.2 equiv.) and slowly warmed up to room temperature. After stirring for 8 hours, the solution was cooled down to $0{ }^{\circ} \mathrm{C}$, trifluoromethanesulfonic acid (14 $\mu 1,0.16 \mathrm{mmol}, 2.0$ equiv.) was added, and the reaction was allowed to warm to room temperature again. After 2 hours at ambient temperature, the reaction mixture was cooled to $0{ }^{\circ} \mathrm{C}$ again and treated with $\mathrm{NaOH}$ (0.32 mL, 10\% in $\mathrm{MeOH}, 10$ equiv.) followed by $\mathrm{H}_{2} \mathrm{O}_{2}(0.08 \mathrm{~mL}, 30 \%$ aq, 10 equiv.). The resulting solution was aged for an additional 6 hours and then partitioned between EtOAc and water $(20 \mathrm{~mL}$, 1:1). The aqueous layer was separated and extracted with EtOAc $(2 \times 5 \mathrm{~mL})$. The combined organic portions were washed with brine $(20 \mathrm{~mL})$, dried over $\mathrm{MgSO}_{4}$, filtered, and concentrated. The resultant material from the first stage was redissolved in EtOAc $(0.8 \mathrm{~mL}, 0.1 \mathrm{M})$. IBX (225 mg, $0.81 \mathrm{mmol}, 10$ equiv.) was added and the suspension was refluxed for 3 hours until conversion was observed (monitoring by TLC). Then the solution was cooled to room temperature, filtered through a short pad of $\mathrm{SiO}_{2}$, and concentrated. The residue was purified by flash chromatography $\left(\mathrm{SiO}_{2}\right.$, hexanes : EtOAc $\left.=10: 1\right)$ and desired triketone 17 (19 mg, $0.062 \mathrm{mmol}$, $80 \%$ ) was isolated as a white crystalline solid. 


$\begin{array}{ll}\mathbf{R}_{\mathbf{f}} & 0.33\left(\mathrm{SiO}_{2}, \text { hexanes }: \text { EtOAc }=3: 1\right) \\ \mathbf{T}_{\text {melt. }} & 134.1-134.8^{\circ} \mathrm{C}\end{array}$

${ }^{1} \mathbf{H}$ NMR $\quad\left(500 \mathrm{MHz}, \mathrm{CDCl}_{3}\right) \delta 13.66(\mathrm{~s}, 1 \mathrm{H}), 2.72(\mathrm{ddd}, J=16.1,12.0,5.8 \mathrm{~Hz}, 1 \mathrm{H}), 2.43(\mathrm{dd}$, $J=13.1,2.6 \mathrm{~Hz}, 1 \mathrm{H}), 2.37(\mathrm{ddd}, J=16.1,9.8,3.1 \mathrm{~Hz}, 1 \mathrm{H}), 2.31-2.22(\mathrm{~m}, 2 \mathrm{H})$, 2.16 (ddd, $J=12.5,12.3,3.1 \mathrm{~Hz}, 1 \mathrm{H}), 2.04$ (dd, $J=13.3,8.7 \mathrm{~Hz}, 1 \mathrm{H}), 1.99$ (s, 3H), $1.93(\mathrm{t}, J=9.3 \mathrm{~Hz}, 1 \mathrm{H}), 1.89$ (ddd, $J=14.1,13.4,8.7 \mathrm{~Hz}, 1 \mathrm{H}), 1.63-1.57(\mathrm{~m}, 1 \mathrm{H})$, $1.55-1.48(\mathrm{~m}, 2 \mathrm{H}), 1.34(\mathrm{~s}, 3 \mathrm{H}), 1.11(\mathrm{~s}, 3 \mathrm{H}), 1.05(\mathrm{~s}, 3 \mathrm{H}), 0.85(\mathrm{~s}, 3 \mathrm{H})$

${ }^{13} \mathrm{C}$ NMR $\quad\left(126 \mathrm{MHz}, \mathrm{CDCl}_{3}\right.$, Major enol form 17): $\delta$ 219.2, 207.3, 172.5, 123.7, 50.7, 47.1, 45.3, 41.3, 35.9, 34.7, 34.6, 33.6, 31.3, 29.3, 26.4, 23.7, 19.5, 19.4, 19.0

(126 MHz, $\mathrm{CDCl}_{3}$, Minor ketone form 17', only distinguishable peaks are listed): $\delta 218.7,210.7,48.9,45.7,42.9,36.0,35.7,34.7,33.3,32.0,31.5,24.2,21.4,19.4$, 18.9

HRMS $\quad(\mathrm{ES}+, m / z)[\mathrm{M}+\mathrm{H}]^{+}$calcd. for $\mathrm{C}_{19} \mathrm{H}_{29} \mathrm{O}_{3}, 305.2117$; found, 305.2118.

IR (ATR, neat, $\left.\mathrm{cm}^{-1}\right): 2935(\mathrm{br}), 1704(\mathrm{~s}), 1650(\mathrm{~m}), 1615(\mathrm{~m}), 1382(\mathrm{w}), 1230(\mathrm{w})$
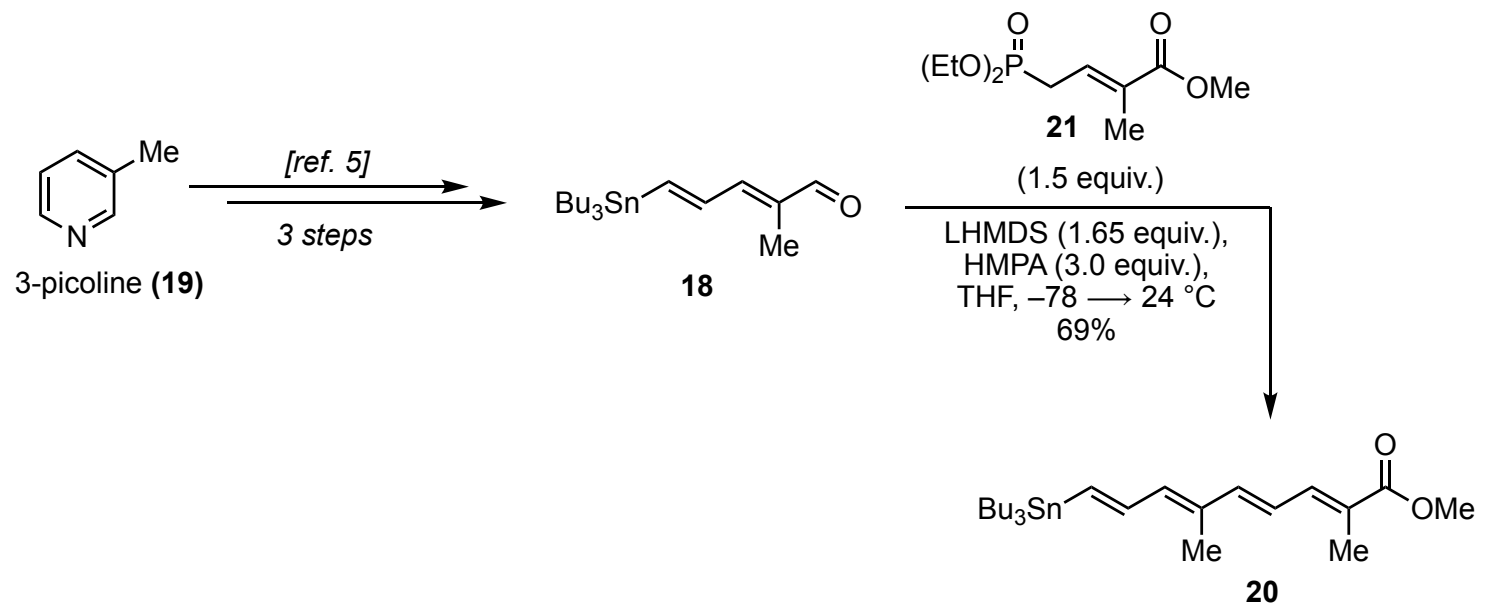

\section{Methyl $(2 E, 4 E, 6 E, 8 E)-2,6-d i m e t h y l-9-(t r i b u t y l s t a n n y l) n o n a-2,4,6,8-t e t r a e n o a t e$ (Stannane 20):}

To a solution of phosphonate $2 \mathbf{1}^{4}$ (195 $\mathrm{mg}, 0.779 \mathrm{mmol}, 1.5$ equiv.) in THF (24 mL, 0.033M) under nitrogen was added a 1.0 M THF solution of LHMDS (143 mg, $0.857 \mathrm{mmol}, 0.860 \mathrm{~mL}, 1.65$ equiv.) dropwise at $-10^{\circ} \mathrm{C}$. After ten minutes, the reaction was cooled to $-60^{\circ} \mathrm{C}$ and $\mathrm{HMPA}(0.271$ $\mathrm{mL}, 1.56 \mathrm{mmol}, 3.0$ equiv.) was added dropwise. After ten minutes, the reaction was cooled to $-78{ }^{\circ} \mathrm{C}$, and a solution of stannanedienal $\mathbf{1 8}^{5}$ in THF $(200 \mathrm{mg}, 0.519 \mathrm{mmol}, 0.55 \mathrm{~mL}, 0.95 \mathrm{M}$, 1.0 equiv.) was added dropwise. The reaction was held at $-78{ }^{\circ} \mathrm{C}$ for 1.5 hours and then warmed to room temperature. After stirring for 30 minutes at room temperature, the reaction was quenched 
with $\mathrm{NH}_{4} \mathrm{Cl}$ (aq.sat. $\left.30 \mathrm{~mL}\right)$, and extracted with $\mathrm{Et}_{2} \mathrm{O}(3 \times 15 \mathrm{~mL})$, washed with brine $(40 \mathrm{~mL})$, and dried over $\mathrm{MgSO}_{4}$. The solvent was removed in vacuo. The material was quickly purified with short plug of silica gel with $10 \%$ of EtOAc in hexanes to remove the excess phosphonate. Stannane 20 was obtained as a bright yellow oil (173 $\mathrm{mg}, 0.519 \mathrm{mmol}, 68 \%$ yield) that was used for the next step without additional purification. For characterization, this material was purified using Biotage ${ }^{\circledR}$ Isolera $^{\mathrm{TM}}$ One (Cartridge Biotage ${ }^{\circledR}$ SNAP Ultra, HP-Sphere C18; $25 \mu \mathrm{m} ; 12 \mathrm{~g}, 100 \% \mathrm{MeCN}$; detection at $\lambda=353 \mathrm{~nm}$ )

$\mathbf{R}_{\mathbf{f}} \quad 0.24\left(\mathrm{SiO}_{2}\right.$, hexanes : EtOAc $\left.=99: 1\right)$

${ }^{1}$ H NMR $\quad\left(500 \mathrm{MHz}, \mathrm{CDCl}_{3}\right) \delta 7.28(\mathrm{~d}, J=11.0 \mathrm{~Hz}, 1 \mathrm{H}), 6.92(\mathrm{dd}, J=18.5,10.6 \mathrm{~Hz}, 1 \mathrm{H})$, $6.53(\mathrm{~m}, 3 \mathrm{H}), 6.19(\mathrm{~d}, J=10.7 \mathrm{~Hz}, 1 \mathrm{H}), 3.76(\mathrm{~s}, 3 \mathrm{H}), 1.99(\mathrm{~s}, 3 \mathrm{H}), 1.97(\mathrm{~s}, 3 \mathrm{H})$, 1.52 (p, $J=8.1,8.1,7.6,7.6 \mathrm{~Hz}, 6 \mathrm{H}), 1.32$ (h, $J=7.3 \mathrm{~Hz}, 6 \mathrm{H}), 0.93$ (m, 6H), 0.90 (t, $J=7.5,7.5 \mathrm{~Hz}, 9 \mathrm{H})$

${ }^{13} \mathrm{C} \mathrm{NMR} \quad\left(126 \mathrm{MHz}, \mathrm{CDCl}_{3}\right): \delta 169.1,144.6,142.8,139.7,139.1,137.6,133.7,126.0,123.6$, $51.9,29.3,27.4,13.9,13.0,12.8,9.8$

HRMS (ES+, $m / z)[\mathrm{M}+\mathrm{Na}]^{+}$calcd. for $\mathrm{C}_{24} \mathrm{H}_{42} \mathrm{NaO}_{2}{ }^{120} \mathrm{Sn}, 505.2104$; found, 505.2125.

IR

(ATR, neat, $\mathrm{cm}^{-1}$ ): 2923 (br), 1705 (s), 1618 (w), 1582 (m), 1284 (m), 1227 (s)
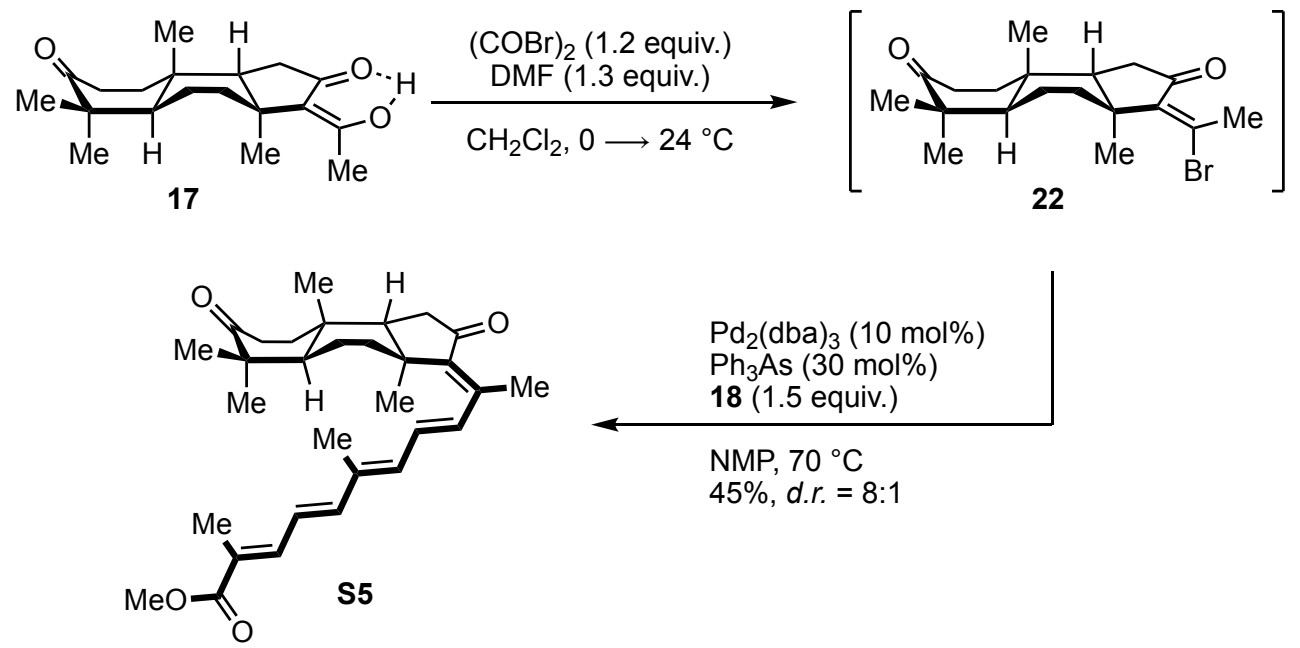

\section{Rhabdastrellic acid A methyl ester (S5):}

A mixture of triketone 17 (5.80 mg, $19.1 \mu \mathrm{mol}, 1.0$ equiv.), DMF (1.9 $\mu \mathrm{L}, 24.8 \mu \mathrm{mol}, 1.30$ equiv.), and $\mathrm{CH}_{2} \mathrm{Cl}_{2}(100 \mu \mathrm{L})$ was cooled to $0{ }^{\circ} \mathrm{C}$, and oxalyl bromide $(2.15 \mu \mathrm{L}, 22.9 \mu$ mol, 1.20 equiv.) was added dropwise, with concurrent gas evolution. The reaction was allowed to warm to ambient temperature while stirring for approximately 2 hours, and then poured into ether and a cold solution 
of $\mathrm{NaHCO}_{3}$. The organic layer was separated, dried over anhydrous sodium sulfate and filtered. Quantitative conversion to a single isomer of vinyl bromide 22 was observed, and this material was used in the next stage directly without additional purification due to instability of the compound on silica gel.

Caution! Product is light-sensitive. All workup and purification procedures were performed with rigorous protection from light, working in a dark room with foil-wrapped, amber glassware.

In a nitrogen-filled glovebox, an amber glass vial was charged with $\mathrm{Pd}_{2}(\mathrm{dba})_{3}(1.7 \mathrm{mg}, 1.9 \mu \mathrm{mol}$, $10 \mathrm{~mol} \%$ ), and $\mathrm{Ph}_{3} \mathrm{As}(1.8 \mathrm{mg}, 5.7 \mu \mathrm{mol}, 30 \mathrm{~mol} \%)$. The vial was placed under an atmosphere of argon, and $100 \mu \mathrm{L}$ of NMP (degassed under nitrogen by 5 freeze-pump-thaw cycles) was added, and the solution was stirred for 5 minutes at room temperature. A solution of crude vinyl bromide (7.0 mg, $19 \mu \mathrm{mol}, 1.0$ equiv.) in $100 \mu \mathrm{L}$ of NMP was added at room temperature, and stirred for ten minutes. At this time, the vessel was shielded from all possible sources of light, and a solution of stannane 20 (14 mg, $29 \mu \mathrm{mol}, 1.5$ equiv.) in $100 \mu \mathrm{L}$ of NMP was added, and the reaction was heated to $70{ }^{\circ} \mathrm{C}$. After two hours, the reaction was cooled to room temperature, $300 \mu \mathrm{L}$ of saturated KF solution was added. The reaction was stirred at room temperature for 30 minutes, after which it was extracted with $\mathrm{Et}_{2} \mathrm{O}(3 \times 5 \mathrm{~mL})$. The combined organic extracts were washed with water $(10 \mathrm{~mL})$, washed with $\mathrm{KF}$ (aq. sat. $10 \mathrm{~mL}$ ), dried with $\mathrm{MgSO}_{4}$, and concentrated in vacuo. The methyl ester S5 was purified by preparative TLC, with two developments in a hexanes : EtOAc $=2: 1$, the desired compound eluting as the bottom bright yellow bands easily visible to the eye. This band was scraped off of the silica, stirred in EtOAc for 40 minutes, filtered, rinsed again with EtOAc, and concentrated in vacuo. Methyl ester S5 was obtained as a bright yellow oil (4.0 mg, 45\%), in an 8:1 mixture with stelletin E methyl ester (S6). These diastereomers were separated for characterization by reverse phase preparative HPLC (Kinetex ${ }^{\circledR} 5 \mu$ m Biphenyl $100 \AA$ LC Column 250 x $10 \mathrm{~mm}, 5 \mathrm{~mL} / \mathrm{min}, \mathrm{MeCN}: \mathrm{H}_{2} \mathrm{O}=4: 3$, detection at $\lambda=374 \mathrm{~nm}$, $\left.t_{R}=30.3 \mathrm{~min}\right)$.

$\mathbf{R}_{\mathbf{f}} \quad 0.40\left(\mathrm{SiO}_{2}\right.$, hexanes : EtOAc $\left.=2: 1\right)$

${ }^{1}$ H NMR $\quad\left(500 \mathrm{MHz}, \mathrm{CDCl}_{3}\right): \delta 7.29(\mathrm{~m}, 1 \mathrm{H}), 7.05(\mathrm{dd}, J=15.0,11.3 \mathrm{~Hz}, 1 \mathrm{H}), 6.62(\mathrm{~m}, 2 \mathrm{H})$, $6.39(\mathrm{~d}, J=11.4 \mathrm{~Hz}, 1 \mathrm{H}), 3.78(\mathrm{~s}, 3 \mathrm{H}), 2.74(\mathrm{~m}, 1 \mathrm{H}), 2.42(\mathrm{~m}, 1 \mathrm{H}), 2.35(\mathrm{~s}, 3 \mathrm{H})$, $2.23(\mathrm{~m}, 2 \mathrm{H}), 2.21-2.14(\mathrm{~m}, 2 \mathrm{H}), 2.04(\mathrm{~s}, 3 \mathrm{H}), 2.02(\mathrm{~s}, 3 \mathrm{H}), 1.88(\mathrm{~m}, 1 \mathrm{H}), 1.71-1.61$ $(\mathrm{m}, 3 \mathrm{H}), 1.57-1.49(\mathrm{~m}, 2 \mathrm{H}), 1.44(\mathrm{~s}, 3 \mathrm{H}), 1.13(\mathrm{~s}, 3 \mathrm{H}), 1.06(\mathrm{~s}, 3 \mathrm{H}), 0.86(\mathrm{~s}, 3 \mathrm{H})$

${ }^{13} \mathbf{C}$ NMR $\quad\left(126 \mathrm{MHz}, \mathrm{CDCl}_{3}\right): \delta 219.3,207.2,169.0,146.7,143.4,141.9,138.8,138.7,135.1$, $134.4,132.1,127.5,125.0,52.1,48.0,47.0,45.6,45.2,38.6,36.9,34.9,33.6,31.5$, $29.4,26.1,23.6,19.9,19.5,14.7,13.2,13.1$ 
HRMS $\quad($ ES,$+ m / z)[\mathrm{M}+\mathrm{H}]^{+}$calcd. for $\mathrm{C}_{31} \mathrm{H}_{43} \mathrm{O}_{4}, 479.3161$; found, 479.3159.

IR (ATR, neat, $\left.\mathrm{cm}^{-1}\right): 2948$ (br), 1702 (s), 1616 (w), 1576 (m), 1266 (m), 1231 (s), $1126(\mathrm{~m})$
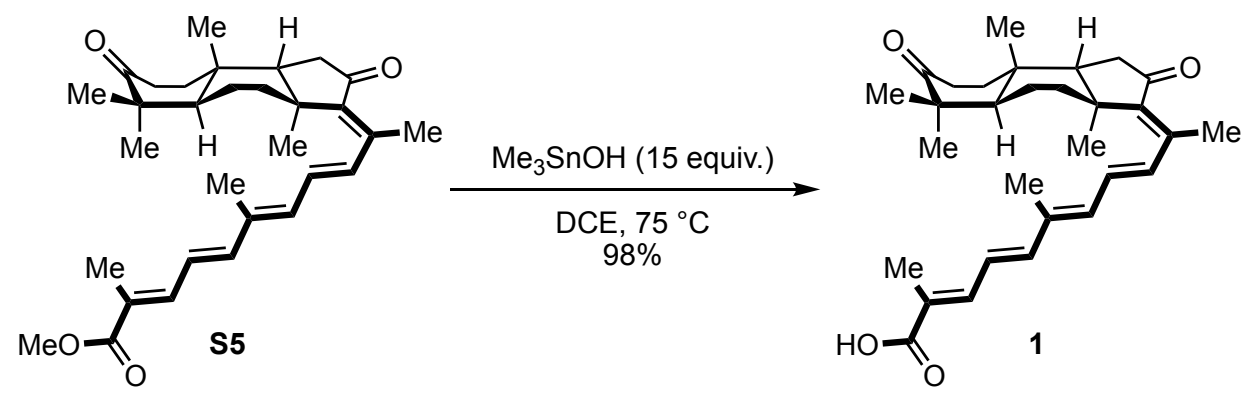

Rhabdastrellic acid A (1):

Caution! Product is light-sensitive. Isomerization into thermodynamic mixture of isomers $(\mathbf{1 : 2}=1.9: 1)$ occurs within several hours upon exposure to ambient light, decomposition observed within $24 \mathrm{~h}$. All workup and purification procedures were performed with rigorous protection from light, working in a dark room with foil-wrapped, amber glassware. Product is thermally stable. A mixture of methyl ester $\mathbf{S 5}$ (13.0 mg, $27.2 \mu \mathrm{mol}, 1.0$ equiv.), trimethyltin hydroxide (24.6 mg, $136.0 \mu \mathrm{mol}, 5.0$ equiv. $)$, in DCE $(0.4 \mathrm{~mL})$ was heated to $75{ }^{\circ} \mathrm{C}$. Two additional portions of trimethyltin hydroxide ( $24.6 \mathrm{mg}, 136.0 \mu \mathrm{mol}, 5.0$ equiv.) were added with 12 hours interval. After 36 hours, full conversion was observed by TLC. The resulting suspension was cooled to ambient temperature, partitioned between $\mathrm{CH}_{2} \mathrm{Cl}_{2}(8 \mathrm{~mL})$ and $1 \mathrm{M} \mathrm{HCl}(8 \mathrm{~mL})$. Organic layer was separated, washed with $1 \mathrm{M} \mathrm{HCl}(3 \times 5 \mathrm{~mL})$, dried over $\mathrm{Na}_{2} \mathrm{SO}_{4}$ and concentrated in vacuo. Resulting mixture was purified by flash chromatography $\left(\mathrm{SiO}_{2}\right.$, hexanes : EtOAc $\left.=3: 1\right)$ that allowed for isolation of rhabdastrellic acid A (1) $(12.3 \mathrm{mg}, 26.5 \mu \mathrm{mol}, 98 \%)$ as yellow solid. Material was further purified by reverse phase preparative HPLC (Kinetex ${ }^{\circledR} 5 \mu \mathrm{m}$ Biphenyl $100 \AA$ LC Column $250 \times 10 \mathrm{~mm}, 4.02 \mathrm{~mL} / \mathrm{min}, \mathrm{MeCN}: \mathrm{H}_{2} \mathrm{O}=1.15: 1$, detection at $\lambda=374 \mathrm{~nm}$, $\left.t_{R}=22.0 \mathrm{~min}\right)$.

$\mathbf{R}_{\mathbf{f}}$

$0.27\left(\mathrm{SiO}_{2}\right.$, hexanes : $\left.\mathrm{EtOAc}=1: 1\right)$

${ }^{1}$ H NMR $\quad\left(500 \mathrm{MHz}, \mathrm{CDCl}_{3}\right): \delta 7.39(\mathrm{dt}, J=9.0,1.6 \mathrm{~Hz}, 1 \mathrm{H}), 7.05(\mathrm{dd}, J=15.0,11.5 \mathrm{~Hz}$, $1 \mathrm{H}), 6.75(\mathrm{~d}, J=15.0 \mathrm{~Hz}, 1 \mathrm{H}), 6.64(\mathrm{~m}, 2 \mathrm{H}), 6.42(\mathrm{~d}, \mathrm{~J}=11.5 \mathrm{~Hz}, 1 \mathrm{H}), 2.74$ (ddd, $\mathrm{J}=16.0,11.9,5.8 \mathrm{~Hz}, 1 \mathrm{H}), 2.42(\mathrm{dd}, J=9.8,3.0 \mathrm{~Hz}, 1 \mathrm{H}), 2.39(\mathrm{~m}, 1 \mathrm{H}), 2.35(\mathrm{~s}$, $3 \mathrm{H}), 2.24(\mathrm{~m}, 2 \mathrm{H}), 2.20(\mathrm{~m}, 2 \mathrm{H}), 2.16(\mathrm{~m}, 1 \mathrm{H}), 2.05(\mathrm{~s}, 3 \mathrm{H}), 2.03(\mathrm{~s}, 3 \mathrm{H}), 1.88(\mathrm{dd}$, $J=12.0,10.2 \mathrm{~Hz}, 1 \mathrm{H}), 1.63(\mathrm{~m}, 1 \mathrm{H}), 1.53(\mathrm{~m}, 2 \mathrm{H}), 1.44(\mathrm{~s}, 3 \mathrm{H}), 1.13(\mathrm{~s}, 3 \mathrm{H}), 1.06$, $(\mathrm{s}, 3 \mathrm{H}), 0.86(\mathrm{~s}, 3 \mathrm{H})$ 
${ }^{13} \mathbf{C}$ NMR $\quad\left(126 \mathrm{MHz}, \mathrm{CDCl}_{3}\right): \delta 219.3,207.2,171.8,146.9,144.4,141.8,140.6,138.6,135.7$, $134.8,132.0,126.3,124.8,48.0,47.0,45.6,45.2,38.7,36.8,34.9,33.6,31.5,29.4$, $26.1,23.6,19.9,19.5,14.7,13.2,12.9$

HRMS $(\mathrm{ES}+, m / z)[\mathrm{M}+\mathrm{H}]^{+}$calcd. for $\mathrm{C}_{30} \mathrm{H}_{41} \mathrm{O}_{4}, 465.3005$; found, 465.3012.

IR (ATR, neat, $\left.\mathrm{cm}^{-1}\right): 2950(\mathrm{~m}), 1690(\mathrm{~s}), 1167(\mathrm{w})$

Table S1. ${ }^{13} \mathrm{C}$ NMR $\left(\mathrm{CDCl}_{3}\right)$ spectroscopic data comparison of natural ${ }^{6}$ and synthetic rhabdastrellic acid A (1).

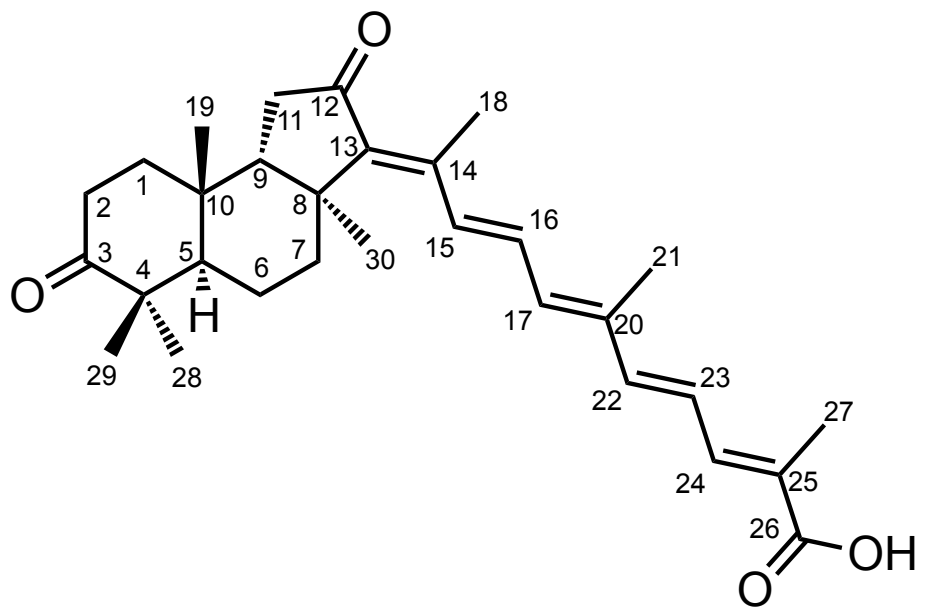

\begin{tabular}{|c|c|c|c|c|c|c|c|}
\hline \# & Natural & Synthetic & $\Delta \delta$ & \# & Natural & Synthetic & $\Delta \delta$ \\
\hline & $\delta^{13} \mathrm{C}(\mathrm{ppm})$ & $\delta{ }^{13} \mathrm{C}(\mathrm{ppm})$ & ppm & & $\delta^{13} \mathrm{C}(\mathrm{ppm})$ & $\delta^{13} \mathrm{C}(\mathrm{ppm})$ & ppm \\
\hline & $150 \mathrm{MHz}$ & $126 \mathrm{MHz}$ & & & $150 \mathrm{MHz}$ & $126 \mathrm{MHz}$ & \\
\hline 1 & 31.4 & 31.5 & -0.1 & 16 & 131.9 & 132.0 & -0.1 \\
\hline 2 & 33.5 & 33.6 & -0.1 & 17 & 135.6 & 135.7 & -0.1 \\
\hline 3 & 219.2 & 219.3 & -0.1 & 18 & 14.6 & 14.7 & -0.1 \\
\hline 4 & 46.9 & 47.0 & -0.1 & 19 & 23.5 & 23.6 & -0.1 \\
\hline 5 & 45.6 & 45.6 & -0.1 & 20 & 138.6 & 138.6 & 0.0 \\
\hline 6 & 19.8 & 19.9 & -0.1 & 21 & 12.7 & 12.9 & -0.3 \\
\hline 7 & 38.6 & 38.7 & -0.1 & 22 & 144.4 & 144.4 & 0.0 \\
\hline 8 & 45.1 & 45.2 & -0.1 & 23 & 124.8 & 124.8 & 0.0 \\
\hline 9 & 47.9 & 48.0 & -0.1 & 24 & 140.6 & 140.6 & 0.0 \\
\hline 10 & 34.9 & 34.9 & 0.0 & 25 & 126.6 & 126.3 & 0.3 \\
\hline 11 & 36.7 & 36.9 & -0.2 & 26 & 173.8 & 171.8 & 2.0 \\
\hline 12 & 207.2 & 207.2 & 0.0 & 27 & 13.1 & 13.2 & -0.1 \\
\hline 13 & 146.8 & 146.9 & -0.1 & 28 & 29.3 & 29.4 & -0.1 \\
\hline 14 & 141.8 & 141.8 & 0.0 & 29 & 19.5 & 19.5 & 0.0 \\
\hline 15 & 134.7 & 134.8 & -0.1 & 30 & 26.0 & 26.1 & -0.1 \\
\hline
\end{tabular}


Table S2. ${ }^{1} \mathrm{H}$ NMR $\left(\mathrm{CDCl}_{3}\right)$ spectroscopic data comparison of natural ${ }^{6}$ and synthetic rhabdastrellic acid A (1).

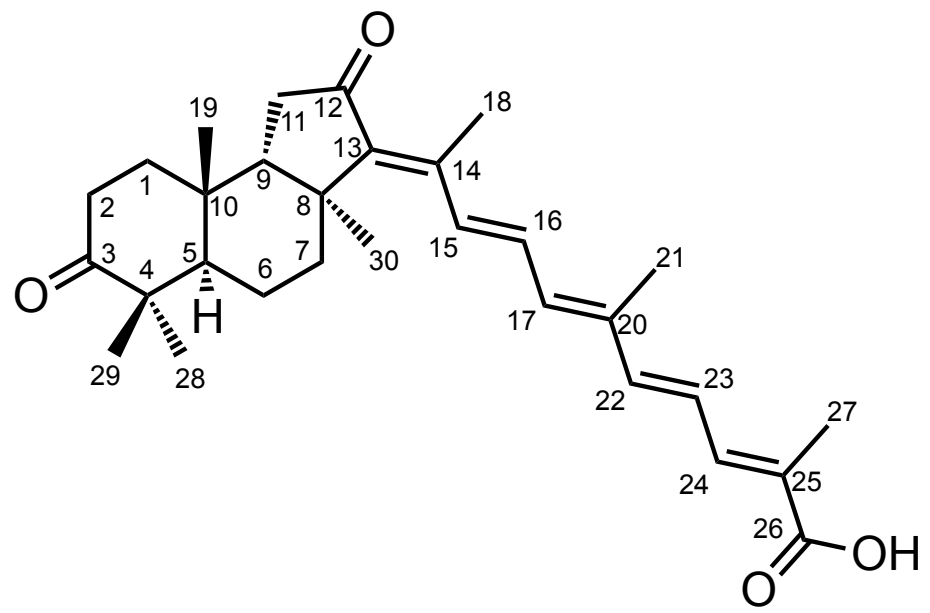

Natural

Synthetic

\begin{tabular}{ccc}
\hline$\#$ & $\delta{ }^{1} \mathrm{H}[\mathrm{ppm}, \mathrm{int}, \mathrm{mult}, \mathrm{J}(\mathrm{Hz})]$ & $\delta{ }^{1} \mathrm{H}[\mathrm{ppm}, \mathrm{int}, \mathrm{mult}, \mathrm{J}(\mathrm{Hz})]$ \\
\hline 24 & $600 \mathrm{MHz}$ & $500 \mathrm{MHz}$ \\
16 & $7.42,1 \mathrm{H}, \mathrm{d}, 9.3$ & $7.39,1 \mathrm{H}, \mathrm{dt}, 9.0,1.6,1.6$ \\
15 & $7.06,1 \mathrm{H}, \mathrm{dd}, 14.5,11.8$ & $7.05,1 \mathrm{H}, \mathrm{dd}, 15.0,11.5$ \\
23,22 & $6.76,1 \mathrm{H}, \mathrm{d}, 15.0$ & $6.75,1 \mathrm{H}, \mathrm{d}, 15.0$ \\
17 & $6.676(1 \mathrm{H}), 6.659(1 \mathrm{H})$ & $6.66,2 \mathrm{H}, \mathrm{m}$ \\
$2 \mathrm{a}$ & $6.44,1 \mathrm{H}, \mathrm{d}, 11.4$ & $6.42,1 \mathrm{H}, \mathrm{d}, 11.5$ \\
5 & $2.76,1 \mathrm{H}, \mathrm{m}$ & $2.74,1 \mathrm{H}, \mathrm{ddd}, 16.0,11.9,5.8$ \\
$2 \mathrm{~b}$ & $2.44,1 \mathrm{H}, \mathrm{m}$ & $2.42,1 \mathrm{H}, \mathrm{dd}, 9.8,3.0$ \\
18 & - & $2.39,1 \mathrm{H}, \mathrm{m}$ \\
11 & $2.37,3 \mathrm{H}, \mathrm{s}$ & $2.24,2 \mathrm{H}, \mathrm{m}$ \\
7 & $2.25,2 \mathrm{H}, \mathrm{dd}, 12.1,10.0$ & $2.20,2 \mathrm{H}, \mathrm{m}$ \\
$1 \mathrm{a}$ & $2.21,2 \mathrm{H}, \mathrm{m}$ & $2.16,1 \mathrm{H}, \mathrm{m}$ \\
21 & - & $2.05,3 \mathrm{H}, \mathrm{s}$ \\
27 & $2.07,3 \mathrm{H}, \mathrm{s}$ & $2.03,3 \mathrm{H}, \mathrm{d}, 1.6$ \\
9 & $2.05,3 \mathrm{H}, \mathrm{s}$ & $1.88,1 \mathrm{H}, \mathrm{dd}, 12.0,10.2$ \\
$6 \mathrm{a}$ & $1.90,1 \mathrm{H}, \mathrm{dd}, 12.1,10$ & $1.63,1 \mathrm{H}, \mathrm{m}$ \\
$6 \mathrm{~b}, 1 \mathrm{~b}$ & $1.64,1 \mathrm{H}, \mathrm{m}$ & $1.53,2 \mathrm{H}, \mathrm{m}$ \\
30 & $1.54,2 \mathrm{H}, \mathrm{m}$ & $1.44,3 \mathrm{H}, \mathrm{s}$ \\
28 & $1.46,3 \mathrm{H}, \mathrm{s}$ & $1.13,3 \mathrm{H}, \mathrm{s}$ \\
29 & $1.15,3 \mathrm{H}, \mathrm{s}$ & $1.06,3 \mathrm{H}, \mathrm{s}$ \\
19 & $1.08,3 \mathrm{H}, \mathrm{s}$ & $0.86,3 \mathrm{H}, \mathrm{s}$ \\
\hline & $0.88,3 \mathrm{H}, \mathrm{s}$ & \\
\hline & &
\end{tabular}



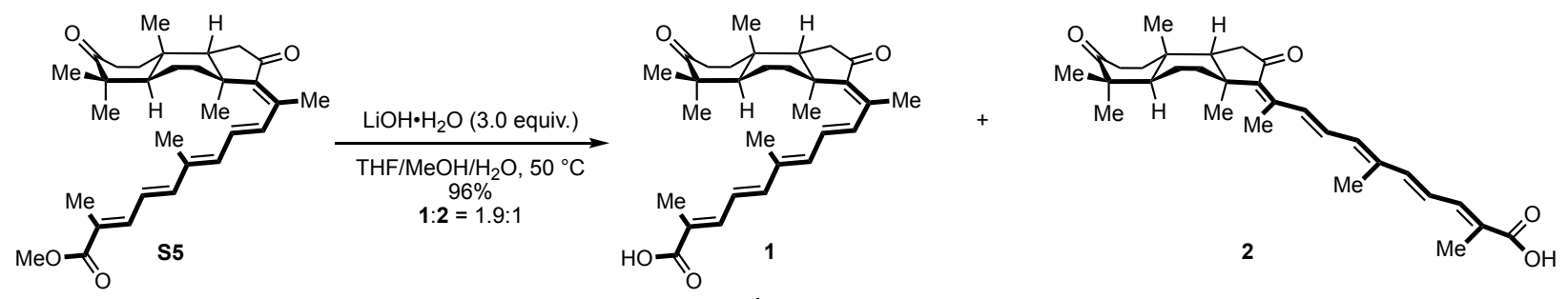

Stelletin E (2):

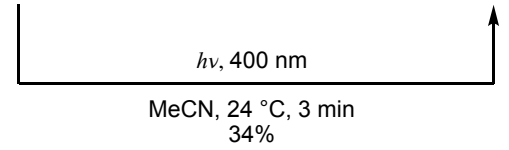

Caution! Product is light-sensitive. Isomerization into thermodynamic mixture of isomers $(\mathbf{1}: \mathbf{2}=1.9: 1)$ occurs within several hours upon exposure to ambient light, decomposition observed within 24 h. All workup and purification procedures were performed with rigorous protection from light, working in a dark room with foil-wrapped, amber glassware. Product is thermally stable.

Synthesis via basic hydrolysis. Methyl ester S5 (14.2 mg, $29.7 \mu \mathrm{mol}, 1.0$ equiv.) in THF: $\mathrm{MeOH}=2: 1(0.3 \mathrm{~mL}, 0.1 \mathrm{M})$ was treated with aqueous solution of $\mathrm{LiOH} \cdot \mathrm{H}_{2} \mathrm{O}(0.2 \mathrm{~mL}, 0.4$ M, 3.0 equiv.). Resulting mixture was heated to $50^{\circ} \mathrm{C}$. After 3 hours, full conversion was observed by TLC. The solution was cooled to ambient temperature, partitioned between EtOAc $(8 \mathrm{~mL})$ and $1 \mathrm{M} \mathrm{HCl}(8 \mathrm{~mL})$. Organic layer was separated, washed with brine $(5 \mathrm{~mL})$, dried over $\mathrm{Na}_{2} \mathrm{SO}_{4}$ and concentrated in vacuo. Resulting yellow solid contained mixture of rhabdastrellic acid $\mathrm{A} /$ stelletin $\mathrm{E}$ in 1.9:1 ratio $(13.2 \mathrm{mg}, 28.4 \mu \mathrm{mol}, 96 \%)$. Isomers were separated by reverse phase preparative HPLC (Kinetex ${ }^{\circledR} 5 \mu \mathrm{m}$ Biphenyl $100 \AA$ LC Column 250 x 10 mm, 4.02 mL/min, $\mathrm{MeCN}: \mathrm{H}_{2} \mathrm{O}=1.15: 1, \quad 35 \mathrm{~min}$, detection at $\lambda=374 \mathrm{~nm}, \quad t_{R}(\mathrm{RhabA})=22.0 \mathrm{~min}$, $\left.t_{R}(\mathrm{StlE})=27.1 \mathrm{~min}\right)$.

Synthesis via photoisomerization. Rhabdastrellic acid A (1) (8.6 mg, $19.0 \mu \mathrm{mol}, 1.0$ equiv.) was dissolved in $\mathrm{MeCN}(7.5 \mathrm{~mL}, 0.025 \mathrm{M})$. Resulting yellow solution was irradiated with ultra violet LED (395-405 $\mathrm{nm}$ range) for $3 \mathrm{~min}$ and isomers were separated by reverse phase preparative HPLC as above to yield stelletin E (2) $(2.8 \mathrm{mg}, 6.0 \mu \mathrm{mol}, 33 \%)$ The recovered rhabdastrellic acid A (1) (5.4 mg, $12.0 \mu \mathrm{mol}, 63 \%)$ was recycled. Overall, stelletin E (2) can be obtained from S5 after hydrolysis and two cycles of photoisomerization in 67\% isolated yield.

$\mathbf{R}_{\mathbf{f}}$

$0.27\left(\mathrm{SiO}_{2}\right.$, hexanes : EtOAc $\left.=1: 1\right)$

${ }^{1}$ H NMR $\quad\left(750 \mathrm{MHz}, \mathrm{CDCl}_{3}\right): \delta 8.15(\mathrm{~d}, J=15.2 \mathrm{~Hz}, 1 \mathrm{H}), 7.39(\mathrm{dd}, J=11.3,1.8 \mathrm{~Hz}, 1 \mathrm{H})$, 6.99 (dd, $J=15.2,11.3 \mathrm{~Hz}, 1 \mathrm{H}), 6.68$ (d, $J=15.0 \mathrm{~Hz}, 1 \mathrm{H}), 6.57$ (dd, $J=15.0$, $11.5 \mathrm{~Hz}, 1 \mathrm{H}), 6.48(\mathrm{~d}, J=11.5 \mathrm{~Hz}, 1 \mathrm{H}), 2.73(\mathrm{ddd}, J=16.8,12.0,5.9 \mathrm{~Hz}, 1 \mathrm{H}), 2.38$ $(\mathrm{d}, J=13.4 \mathrm{~Hz}, 1 \mathrm{H}), 2.37(\mathrm{~m}, 1 \mathrm{H}), 2.24(\mathrm{~m}, 2 \mathrm{H}), 2.18(\mathrm{dd}, J=13.6,8.4 \mathrm{~Hz}, 1 \mathrm{H})$, $2.16(\mathrm{dd}, J=13.2,12.4 \mathrm{~Hz}, 1 \mathrm{H}), 2.09$ (m, 1H), 2.05 (s, 3H), 2.01 (s, 3H), 2.00 (s, 
$3 \mathrm{H}), 1.88(\mathrm{dd}, J=12.5,9.7 \mathrm{~Hz}, 1 \mathrm{H}), 1.61(\mathrm{dd}, J=13.7,8.4 \mathrm{~Hz}, 1 \mathrm{H}), 1.52(\mathrm{~m}, 1 \mathrm{H})$, 1.50 (ddd, $J=13.2,9.9,5.9 \mathrm{~Hz}, 1 \mathrm{H}), 1.40$ (s, 3H), 1.11 (s, 3H), 1.05 (s, 3H), 0.85 (s, 3H)

${ }^{13}$ C NMR $\quad\left(126 \mathrm{MHz}, \mathrm{CDCl}_{3}\right): \delta 219.2,206.2,171.4,146.3,144.9,142.6,140.9,138.0,136.5$, $135.0,130.6,125.6,124.1,48.1,47.0,45.5,45.1,37.3,37.0,34.9,33.6,31.5,29.4$, $24.8,23.6,19.8,19.5,16.1,13.1,12.8$

HRMS $\quad(\mathrm{ES}+, \mathrm{m} / z)[\mathrm{M}+\mathrm{H}]^{+}$calcd. for $\mathrm{C}_{30} \mathrm{H}_{41} \mathrm{O}_{4}, 465.3005$; found, 465.3005.

IR $\quad\left(A T R\right.$, neat, $\left.\mathrm{cm}^{-1}\right): 2957(\mathrm{~m}), 1688(\mathrm{~s}), 1244(\mathrm{w}), 1174(\mathrm{~m})$

Table S3. ${ }^{13} \mathrm{C}$ NMR $\left(\mathrm{CDCl}_{3}\right)$ spectroscopic data comparison of natural ${ }^{7}$ and synthetic stelletin E (2).

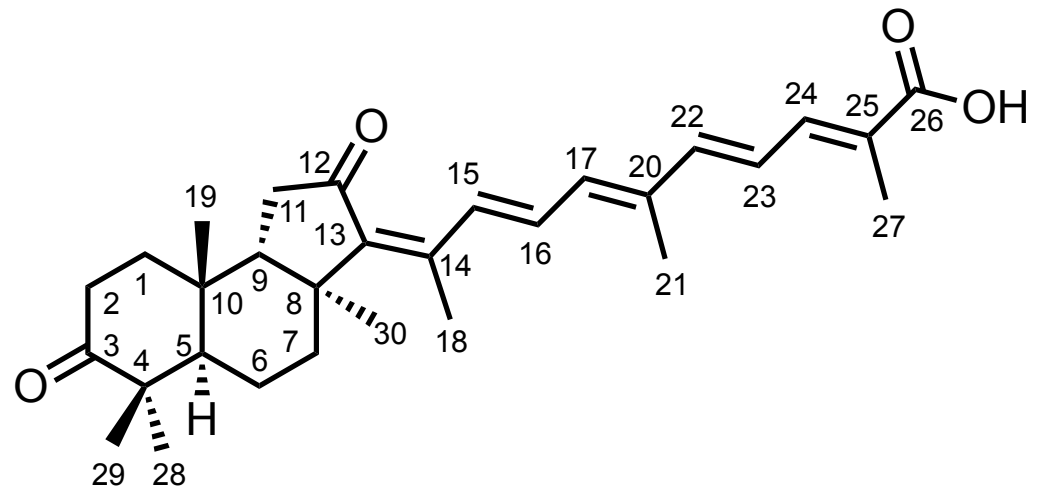

\begin{tabular}{|c|c|c|c|c|c|c|c|}
\hline \# & Natural & Synthetic & $\Delta \delta$ & \# & Natural & Synthetic & $\Delta \delta$ \\
\hline & $\begin{array}{c}\delta^{13} \mathrm{C}(\mathrm{ppm}) \\
150 \mathrm{MHz}\end{array}$ & $\begin{array}{c}\delta^{13} \mathrm{C}(\mathrm{ppm}) \\
126 \mathrm{MHz}\end{array}$ & ppm & & $\begin{array}{c}\delta^{13} \mathrm{C}(\mathrm{ppm}) \\
150 \mathrm{MHz}\end{array}$ & $\begin{array}{c}\delta^{13} \mathrm{C}(\mathrm{ppm}) \\
126 \mathrm{MHz}\end{array}$ & ppm \\
\hline 1 & 31.3 & 31.5 & -0.2 & 16 & 130.5 & 130.6 & -0.1 \\
\hline 2 & 33.5 & 33.6 & -0.1 & 17 & 136.3 & 136.5 & -0.2 \\
\hline 3 & 219.1 & 219.2 & -0.1 & 18 & 15.9 & 16.1 & -0.2 \\
\hline 4 & 46.8 & 47.0 & -0.2 & 19 & 23.5 & 23.6 & -0.1 \\
\hline 5 & 45.4 & 45.5 & -0.1 & 20 & 137.9 & 138.0 & -0.1 \\
\hline 6 & 19.7 & 19.8 & -0.1 & 21 & 12.9 & 13.1 & -0.2 \\
\hline 7 & 37.2 & 37.3 & -0.1 & 22 & 144.7 & 144.9 & -0.2 \\
\hline 8 & 44.9 & 45.1 & -0.2 & 23 & 124.0 & 124.1 & -0.1 \\
\hline 9 & 47.9 & 48.1 & -0.2 & 24 & 140.6 & 140.9 & -0.3 \\
\hline 10 & 34.8 & 34.9 & -0.1 & 25 & 126.0 & 125.6 & 0.4 \\
\hline 11 & 36.8 & 37.0 & -0.2 & 26 & 172.9 & 171.4 & 1.5 \\
\hline 12 & 206.1 & 206.2 & -0.1 & 27 & 12.6 & 12.8 & -0.2 \\
\hline 13 & 146.1 & 146.3 & -0.2 & 28 & 29.2 & 29.4 & -0.2 \\
\hline 14 & 142.6 & 142.6 & 0.0 & 29 & 19.4 & 19.5 & -0.1 \\
\hline 15 & 134.9 & 135.0 & -0.1 & 30 & 24.7 & 24.8 & -0.1 \\
\hline
\end{tabular}


Table S4. ${ }^{1} \mathrm{H}$ NMR $\left(\mathrm{CDCl}_{3}\right)$ spectroscopic data comparison of natural ${ }^{7}$ and synthetic stelletin E (2).

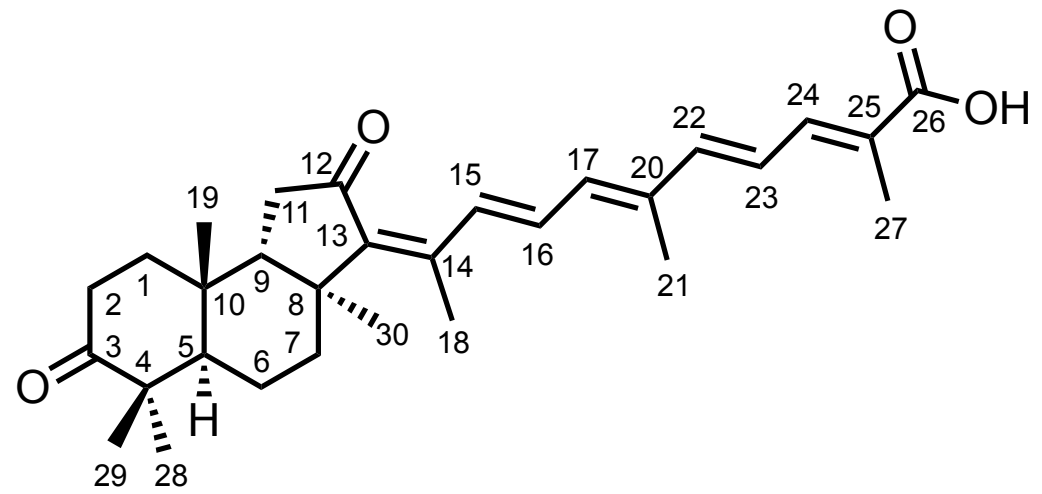

Natural

Synthetic

\begin{tabular}{|c|c|c|}
\hline \# & $\begin{array}{c}\delta{ }^{1} \mathrm{H}[\mathrm{ppm}, \text { int, mult, } \mathrm{J}(\mathrm{Hz})] \\
500 \mathrm{MHz}\end{array}$ & $\begin{array}{c}\delta{ }^{1} \mathrm{H}[\mathrm{ppm}, \text { int, mult, } \mathrm{J}(\mathrm{Hz})] \\
750 \mathrm{MHz}\end{array}$ \\
\hline 15 & $8.12,1 \mathrm{H}, \mathrm{d}, 14.7$ & $8.15,1 \mathrm{H}, \mathrm{d}, 15.2$ \\
\hline 24 & $7.36,1 \mathrm{H}, \mathrm{d}, 11.0$ & $7.39,1 \mathrm{H}, \mathrm{dd}, 11.3,1.8$ \\
\hline 16 & $6.99,1 \mathrm{H}, \mathrm{dd}, 14.7,11.0$ & $6.99,1 \mathrm{H}, \mathrm{dd}, 15.2,11.3$ \\
\hline 22 & $6.641 \mathrm{H}, \mathrm{d}, 14.7$ & $6.68,1 \mathrm{H}, \mathrm{d}, 15.0$ \\
\hline 23 & $6.56,1 \mathrm{H}, \mathrm{dd}, 14.7,11.0$ & $6.57,1 \mathrm{H}, \mathrm{dd}, 15.0,11.5$ \\
\hline 17 & $6.44,1 \mathrm{H}, \mathrm{d}, 11.0$ & $6.48,1 \mathrm{H}, \mathrm{d}, 11.5$ \\
\hline $2 b$ & $2.72,1 \mathrm{H}, \mathrm{m}$ & $2.73,1 \mathrm{H}, \mathrm{ddd}, 16.9,12.0,5.9$ \\
\hline 5 & $2.36,1 \mathrm{H}, \mathrm{m}$ & $2.38,1 \mathrm{H}, \mathrm{d}, 13.4$ \\
\hline $2 \mathrm{a}$ & $2.34,1 \mathrm{H}, \mathrm{m}$ & $2.37,1 \mathrm{H}, \mathrm{m}$ \\
\hline 11 & $2.20,2 \mathrm{H}, \mathrm{m}$ & $2.24,2 \mathrm{H}, \mathrm{m}$ \\
\hline $1 b$ & $2.14,1 \mathrm{H}, \mathrm{m}$ & $2.16,1 \mathrm{H}, \mathrm{dd}, 13.2,12.4$ \\
\hline (7a) & - & $2.18,1 \mathrm{H}, \mathrm{dd}, 13.6,8.4$ \\
\hline 18 & $2.05,3 \mathrm{H}, \mathrm{s}$ & $2.05,3 \mathrm{H}, \mathrm{s}$ \\
\hline (7a) & $2.04,1 \mathrm{H}, \mathrm{m}$ & - \\
\hline $7 b$ & $2.04,1 \mathrm{H}, \mathrm{m}$ & $2.09,1 \mathrm{H}, \mathrm{m}$ \\
\hline 21 & $1.98,3 \mathrm{H}, \mathrm{s}$ & $2.01,3 \mathrm{H}, \mathrm{s}$ \\
\hline 27 & $1.97,3 \mathrm{H}, \mathrm{s}$ & $2.00,3 \mathrm{H}, \mathrm{s}$ \\
\hline 9 & $1.85,1 \mathrm{H}, \mathrm{m}$ & $1.88,1 \mathrm{H}, \mathrm{dd}, 12.5,9.7$ \\
\hline $6 b$ & $1.59,1 \mathrm{H}, \mathrm{m}$ & $1.61,1 \mathrm{H}, \mathrm{dd}, 13.7,8.4$ \\
\hline $6 a$ & $1.50,1 \mathrm{H}, \mathrm{m}$ & $1.52,1 \mathrm{H}, \mathrm{m}$ \\
\hline $1 \mathrm{a}$ & $1.48,1 \mathrm{H}, \mathrm{m}$ & $1.50,1 \mathrm{H}, \mathrm{ddd}, 13.2,9.9,5.9$ \\
\hline 30 & $1.38,3 \mathrm{H}, \mathrm{s}$ & $1.40,3 \mathrm{H}, \mathrm{s}$ \\
\hline 28 & $1.09,3 \mathrm{H}, \mathrm{s}$ & $1.11,3 \mathrm{H}, \mathrm{s}$ \\
\hline 29 & $1.03,3 \mathrm{H}, \mathrm{s}$ & $1.05,3 \mathrm{H}, \mathrm{s}$ \\
\hline 19 & $0.83,3 \mathrm{H}, \mathrm{s}$ & $0.85,3 \mathrm{H}, \mathrm{s}$ \\
\hline
\end{tabular}




\section{Proposed Rationale for $(E)$-selectivity of Vinylogous Acyl Bromide Formation}

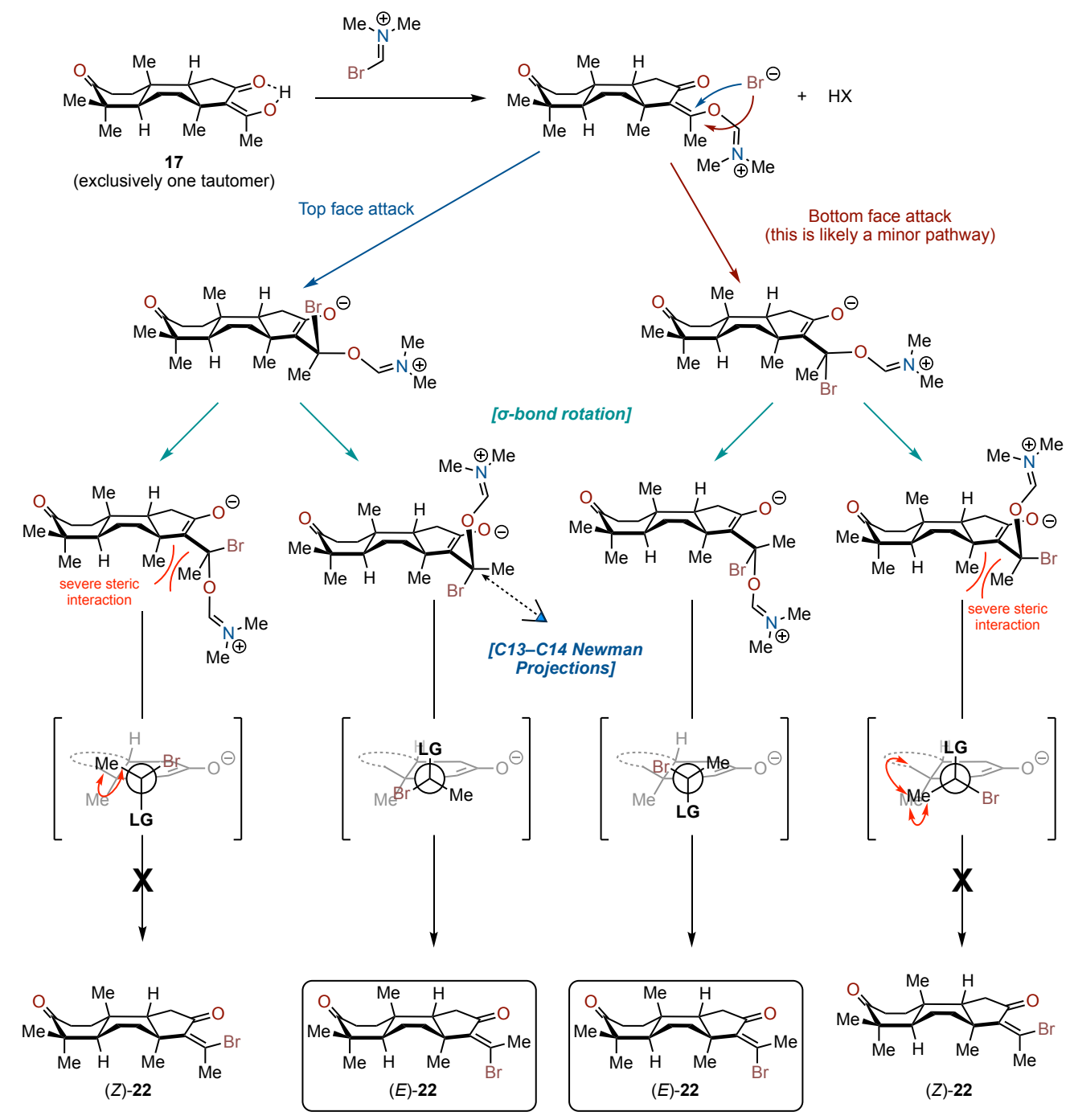




\section{Crystallographic Data \\ Enyne 6}

Crystals were grown from acetonitrile. A suitable crystal was selected and mounted on a CryoLoop (Hampton research) using Krytox oil with $(1-55)$ face roughly perpendicular to the spindle axis. Data were acquired and integrated with APEX3 software with on a Bruker D8 Venture diffractometer with Photon II detector. The crystal was kept at $100.02 \mathrm{~K}$ during data collection. Multi-scan absorption correction was done with SADABS. Using Olex2 [1], the structure was solved with the XT [2] structure solution program using Intrinsic Phasing and refined with the XL [3] refinement package using Least Squares minimization. Hydrogen atoms were added geometrically and refined as riding atoms. Disorder in the main residue was due to the free rotation of the triisopropylsilyl group. Atomic positions with larger occupancies $(0.599(4))$ were found in difference maps and refined. Positions with lower occupancies (0.401(4)) were then revealed. Chemically equivalent distances were restrained using SADI 0.01 instruction. SIMU 0.01 0.02 instruction was applied to the spatially close carbon atoms.

$$
\begin{aligned}
& \text { Identification code dd81u_0m } \\
& \text { Empirical formula } \quad \mathrm{C}_{31} \mathrm{H}_{54} \mathrm{O}_{3} \mathrm{Si} \\
& \text { Formula weight } 502.83 \\
& \text { Temperature / K } \quad 100.0 \\
& \text { Crystal system monoclinic } \\
& \text { Space group } P 22_{1} / n \\
& a / \AA \quad 7.2188(3) \\
& b / \AA \quad 10.7692(4) \\
& \text { c / } \AA \text { 39.2149(14) } \\
& \alpha /{ }^{\circ} \quad 90 \\
& \beta / \circ 93.0050(10) \\
& \gamma / \circ 90 \\
& \text { Volume / } \AA^{3} \quad 3044.4(2) \\
& \text { Z } 4 \\
& \rho_{\text {calc } / \mathrm{g} \mathrm{cm}^{-3} \quad 1.097} \\
& \mu / \mathrm{mm}^{-1} \quad 0.105 \\
& F(000) \quad 1112.0 \\
& \text { Crystal size } / \mathrm{mm}^{3} \quad 0.684 \times 0.152 \times 0.116 \\
& \text { Radiation } \operatorname{MoK} \alpha(\lambda=0.71073) \\
& 2 \Theta \text { range for data collection } /^{\circ} \quad 4.316 \text { to } 50.712 \\
& \text { Index ranges } \quad-8 \leq h \leq 8,-12 \leq k \leq 12,-47 \leq l \leq 47 \\
& \text { Reflections collected } 68464 \\
& \text { Independent reflections } 5659\left[R_{\text {int }}=0.0804, R_{\text {sigma }}=0.0377\right] \\
& \text { Data/restraints/parameters } \quad 5659 / 117 / 421 \\
& \text { Goodness-of-fit on } \mathrm{F}^{2} \quad 1.102 \\
& \text { Final R indexes }[I \geq 2 \sigma(I)] \quad R_{1}=0.0613, w R_{2}=0.1804 \\
& \text { Final } \mathrm{R} \text { indexes [all data] } \quad R_{1}=0.0693, w R_{2}=0.1897 \\
& \text { Largest diff. peak/hole / e } \AA^{-3} \quad 0.59 \text { / }-0.46
\end{aligned}
$$




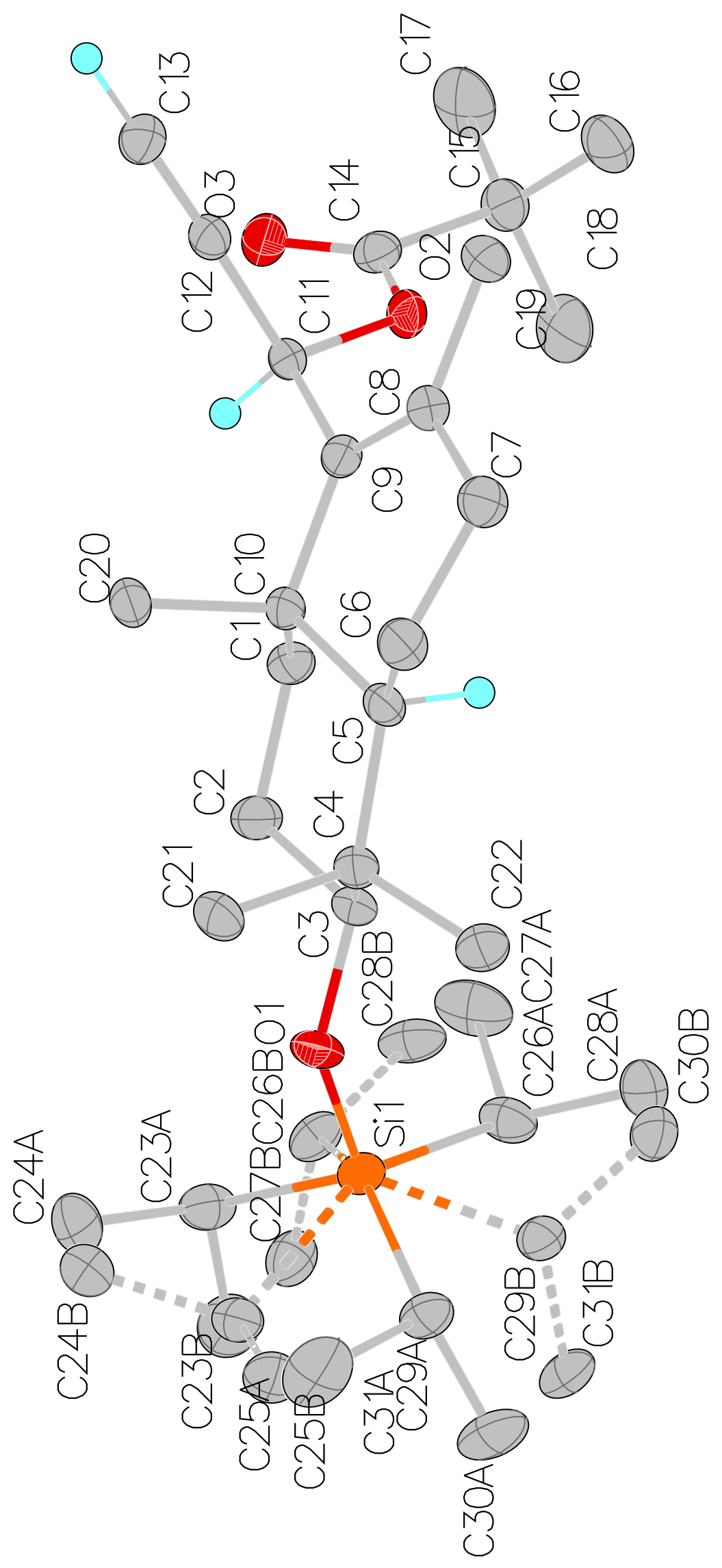




\section{Triketone 17}

Crystals were grown from acetonitrile. A suitable crystal was selected and mounted on a CryoLoop (Hampton research) using Paratone-N oil (Exxon). Data were acquired and integrated with APEX3 software with on a Bruker D8 Venture diffractometer with Photon 100 detector. The crystal was kept at $100.02 \mathrm{~K}$ during data collection. Multi-scan absorption correction was done with SADABS. Using Olex2 [1], the structure was solved with the XT [2] structure solution program using Intrinsic Phasing and refined with the XL [3] refinement package using Least Squares minimization. Hydrogen atoms were added geometrically.

\begin{tabular}{|c|c|}
\hline Identification code & mo_dd16q_0m \\
\hline Empirical formula & $\mathrm{C}_{19} \mathrm{H}_{28} \mathrm{O}_{3}$ \\
\hline Formula weight & 304.41 \\
\hline Temperature / K & 99.99 \\
\hline Crystal system & triclinic \\
\hline Space group & $P-1$ \\
\hline$a / \AA$ & $6.6780(3)$ \\
\hline$b / \AA$ & $9.4722(4)$ \\
\hline $\mathrm{c} / \AA$ & $13.5770(6)$ \\
\hline$\alpha /{ }^{\circ}$ & $72.958(2)$ \\
\hline$\beta /{ }^{\circ}$ & $83.610(2)$ \\
\hline$\gamma / \circ$ & $77.293(2)$ \\
\hline Volume / $\AA^{3}$ & $799.99(6)$ \\
\hline$Z$ & 2 \\
\hline$\rho_{\text {calc } / \mathrm{g} \mathrm{cm}^{-3}}$ & 1.264 \\
\hline$\mu / \mathrm{mm}^{-1}$ & 0.083 \\
\hline$F(000)$ & 332.0 \\
\hline Crystal size $/ \mathrm{mm}^{3}$ & $0.525 \times 0.223 \times 0.124$ \\
\hline Radiation & $\operatorname{MoK} \alpha(\lambda=0.71073)$ \\
\hline $2 \Theta$ range for data collection $/{ }^{\circ}$ & 4.788 to 56.74 \\
\hline Index ranges & $-8 \leq h \leq 8,-12 \leq k \leq 12,-18 \leq l \leq 18$ \\
\hline Reflections collected & 31524 \\
\hline Independent reflections & $3984\left[R_{\text {int }}=0.0438, R_{\text {sigma }}=0.0226\right]$ \\
\hline Data/restraints/parameters & $3984 / 0 / 209$ \\
\hline Goodness-of-fit on $\mathrm{F}^{2}$ & 1.035 \\
\hline Final $\mathrm{R}$ indexes $[I \geq 2 \sigma(I)]$ & $R_{1}=0.0427, w R_{2}=0.1018$ \\
\hline Final $\mathrm{R}$ indexes [all data] & $R_{1}=0.0507, w R_{2}=0.1074$ \\
\hline Largest diff. peak/hole / e $\AA^{-3}$ & $0.39 /-0.27$ \\
\hline
\end{tabular}




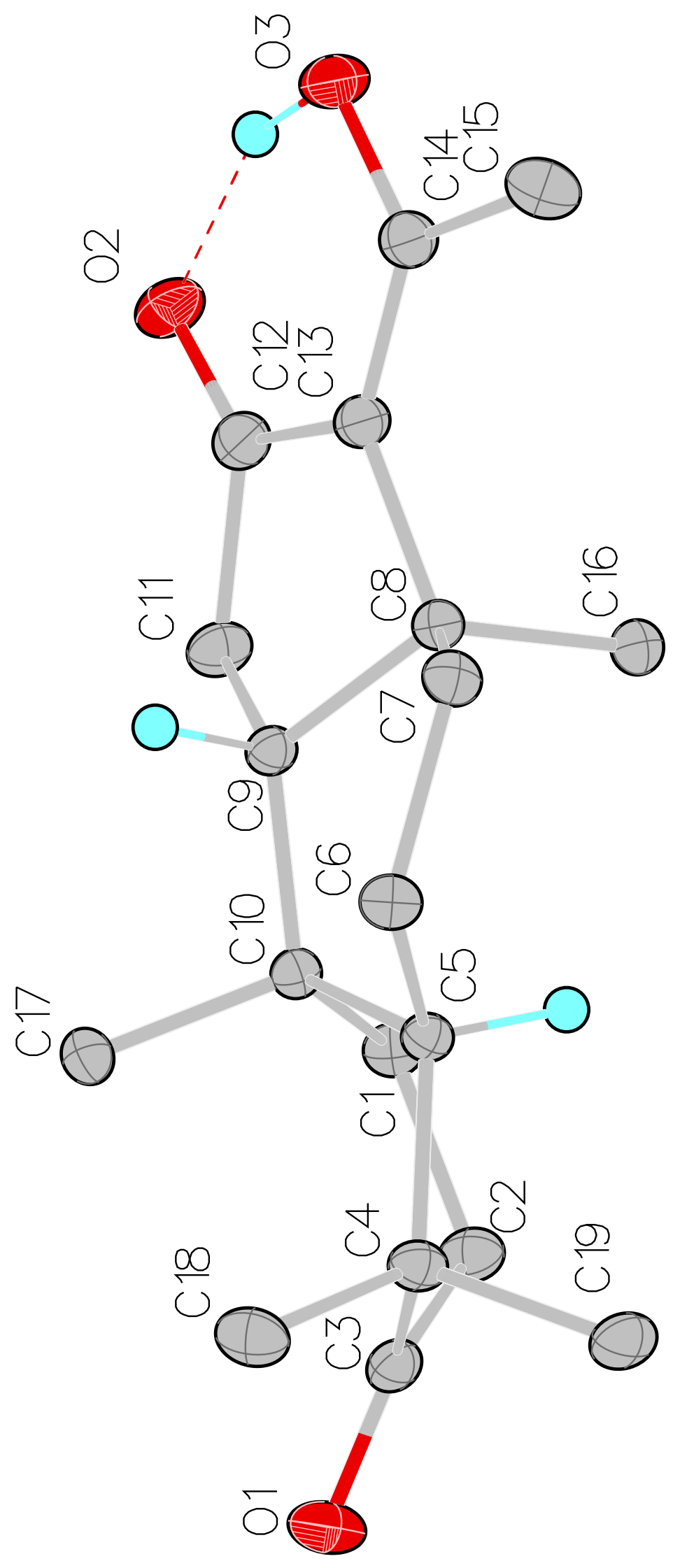




\section{References}

1. Zalesskiy, S. S.; Ananikov, V. P. $\mathrm{Pd}_{2}(\mathrm{dba})_{3}$ as a Precursor of Soluble Metal Complexes and Nanoparticles: Determination of Palladium Active Species for Catalysis and Synthesis Organometallics 2012, 31, 2302-2309.

2. O'Neil, I. A. Reverse Phase Flash Chromatography: A Convenient Method for the Large-Scale Separation of Polar Compounds Synlett 1991, 9, 661-662.

3. Rosenau, C. P.; Jelier, B. J.; Gossert, A. D.; Togni, A. Exposing the Origins of Irreproducibility in Fluorine NMR Spectroscopy Angew. Chem. Int. Ed. 57, 9528-9533 (2018).

4. Wang, J.; Boyarskih, V.; Rainier, J.D. Vinyl Diazophosphonates as Precursors to Quaternary Substituted Indolines and Cyclopentenes. Org. Lett. 2011, 13, 700-702.

5. Michels, T. D.; Rhee, J. U.; Vanderwal, C. D. Synthesis of $\delta$-Tributylstannyl- $\alpha, \beta, \gamma, \delta-$ Unsaturated Aldehydes from Pyridines. Org. Lett. 2008, 10, 4787-4790.

6. Rao, Z.; Deng, S.; Wu, H.; Jiang, S. Rhabdastrellic Acid A: a Novel Triterpenoid from the Marine Sponge Rhabdastrella globostellata. J. Nat. Prod. 1997, 60, 1163-1164.

7. Tasdemir, D.; Mangalindan, G. C.; Concepción, G. P.; Verbitski, S. M.; Rabindran, S.; Miranda, M.; Greenstein, M.; Hooper, J. N. A.; Harper, M. K.; Ireland, C. M. Bioactive Isomalabaricane Triterpenes from the Marine Sponge Rhabdastrella globostellata J. Nat. Prod. 2002, 65, 210-214.

8. Sakamoto, I.; Nishii, T.; Ozaki, F.; Kaku, H.; Masami, T.; Tsunoda, T. Preparation of (Cyanomethylene)tributylphosphorane: A New Mitsunobu-Type Reagent Chem. Pharm. Bull. 2005, 53, 1508-1509.

9. Zi, W.; Wu, H.; Toste F. D. Gold(I)-Catalyzed Dearomative Rautenstrauch Rearrangement: Enantioselective Access to Cyclopenta[b]indoles J. Am. Chem. Soc. 2015, 137, 3225-3228.

10. Dolomanov, O.V., Bourhis, L.J., Gildea, R.J, Howard, J.A.K.; Puschmann, H. J. Appl. Cryst. 2009, 42, 339-341.

11. Sheldrick, G.M. A short history of SHELX Acta Cryst. 2008, A64, 112-122. 
NMR Spectral Data 


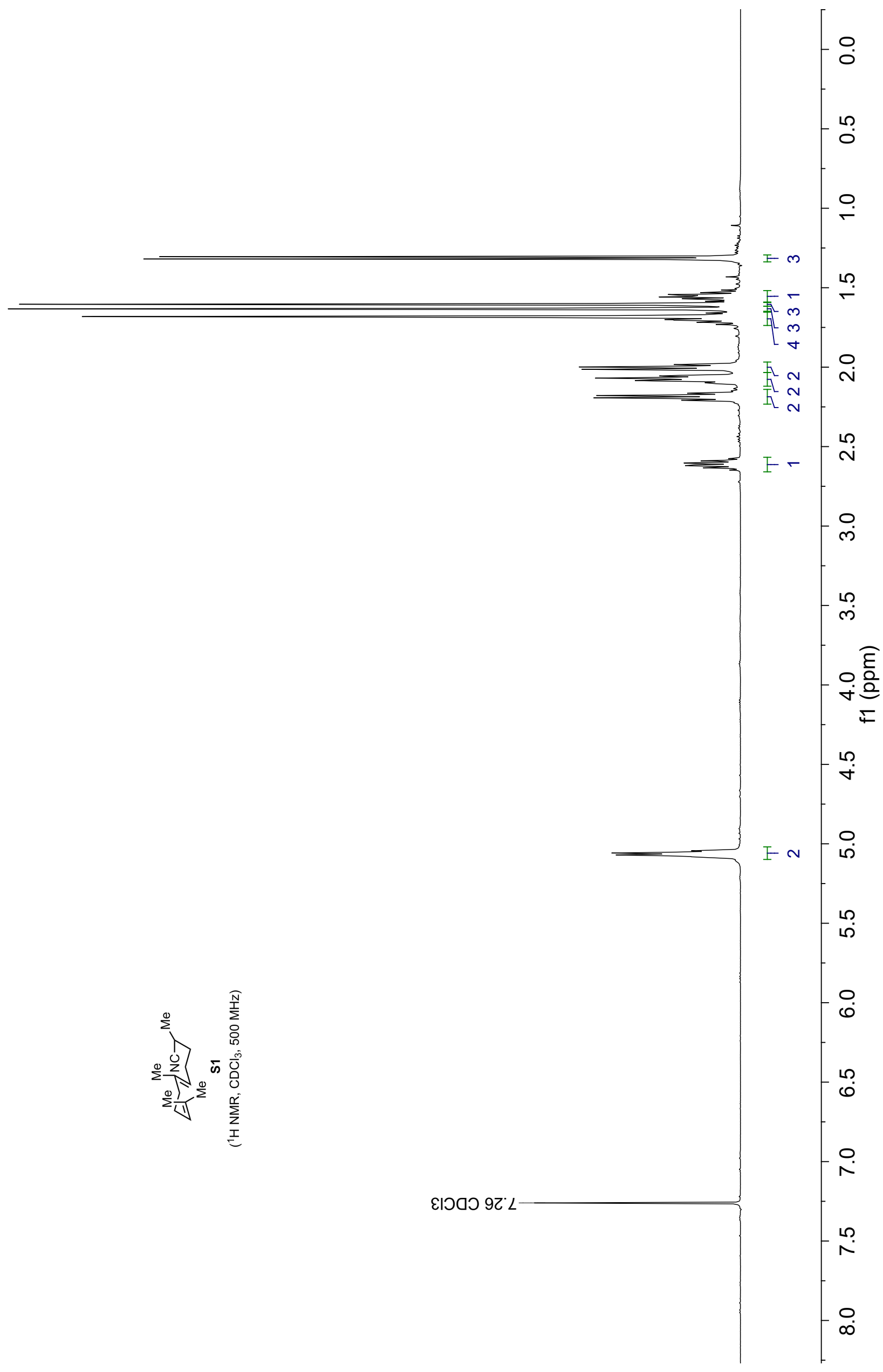




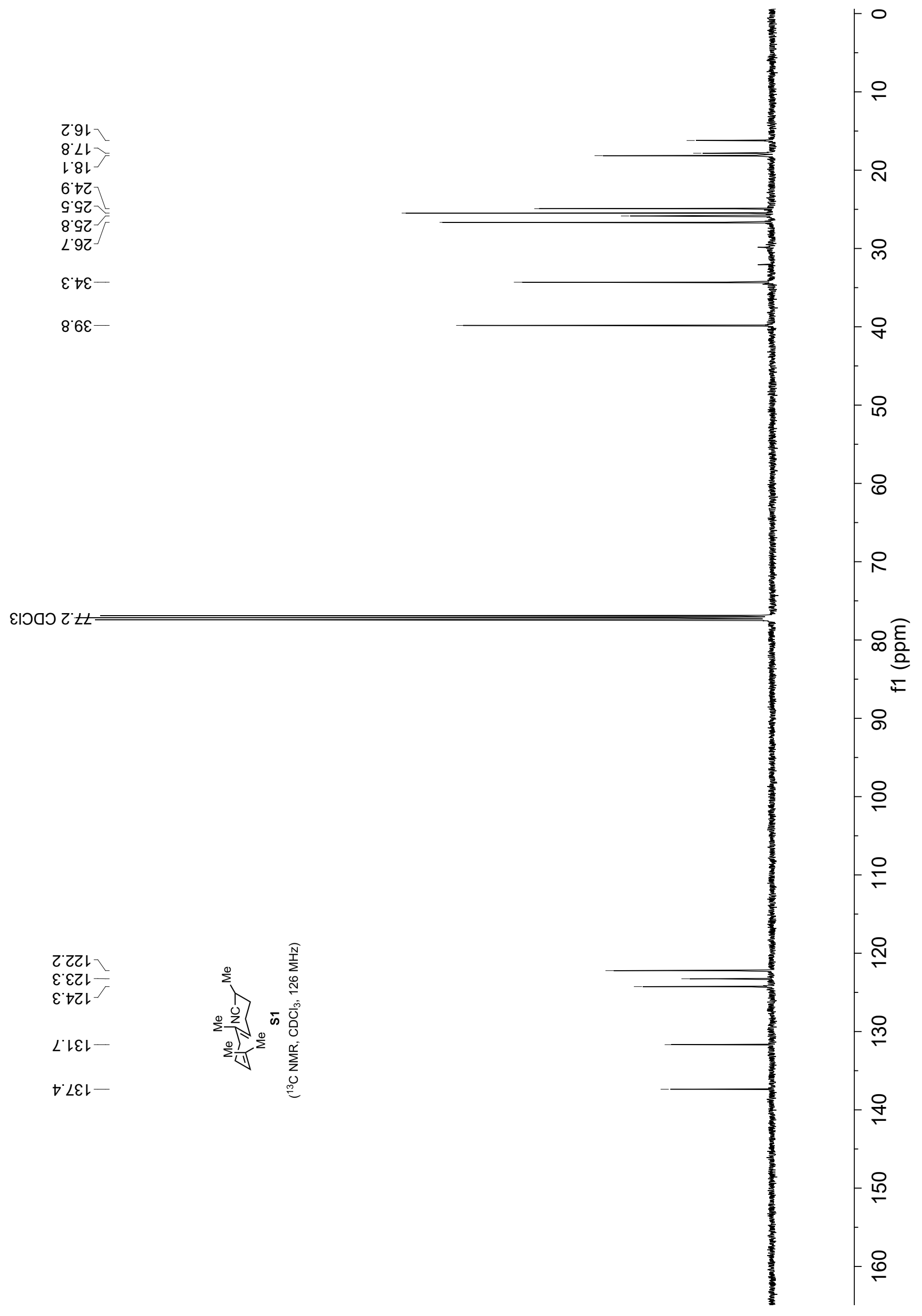




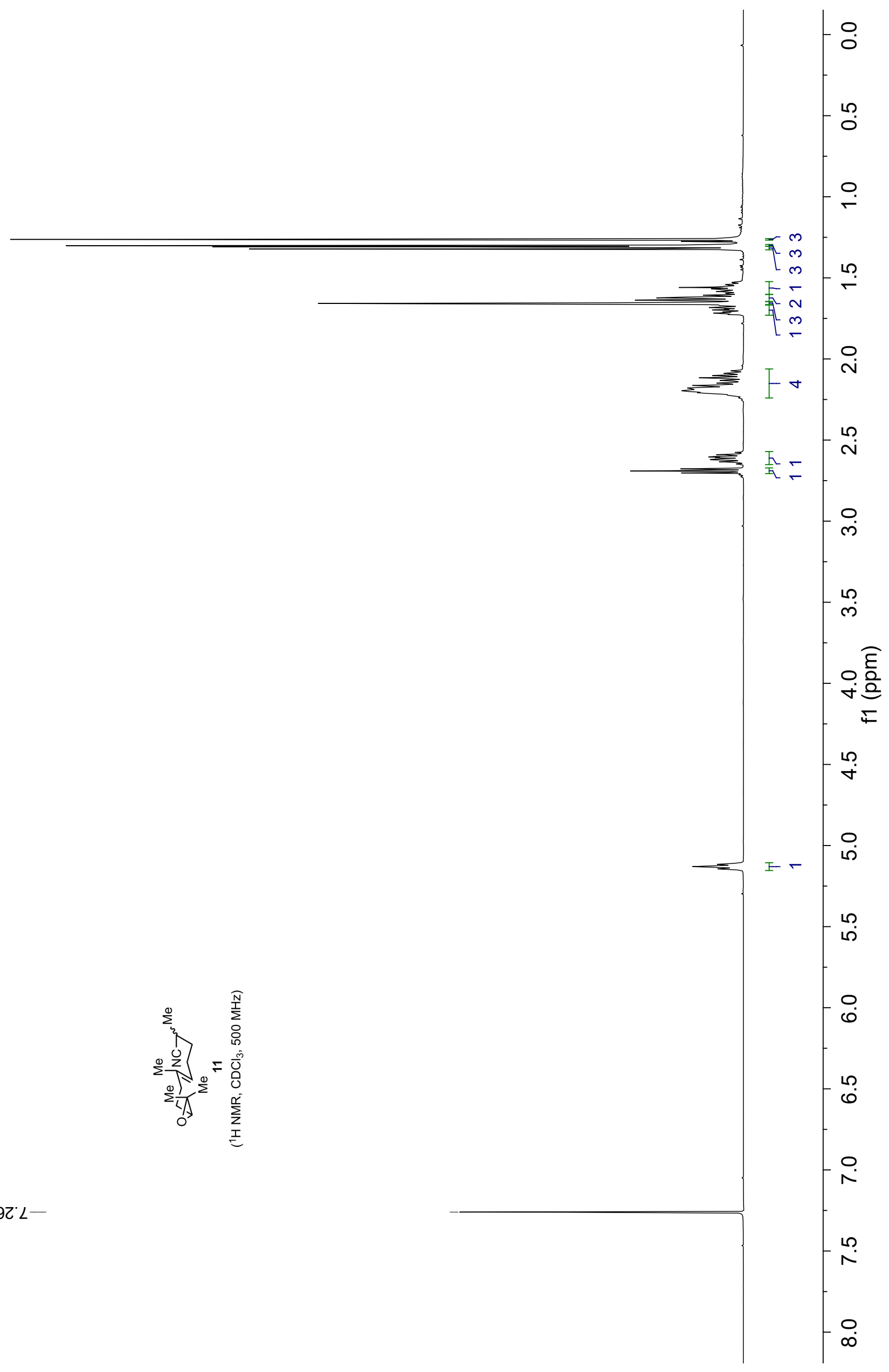




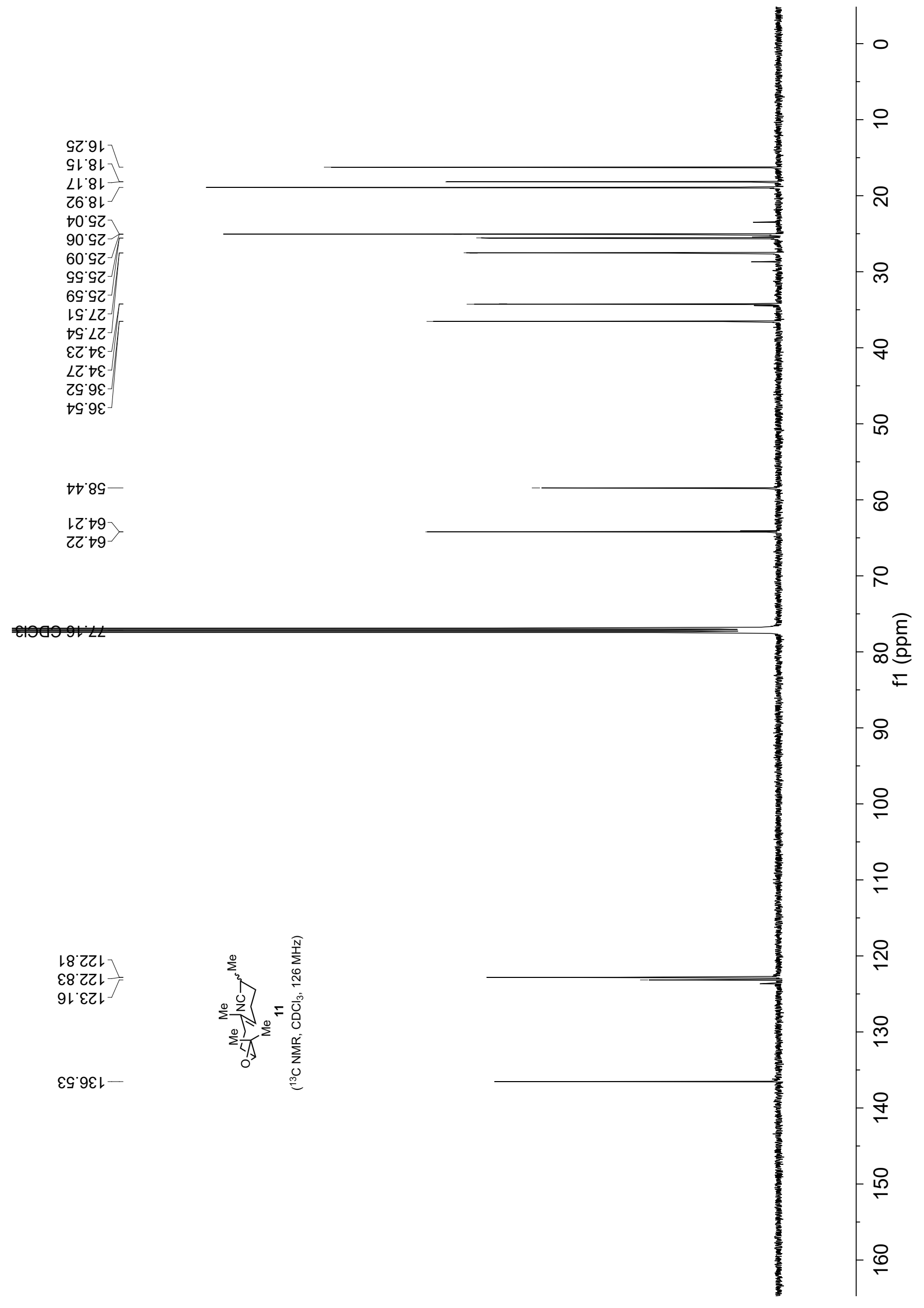




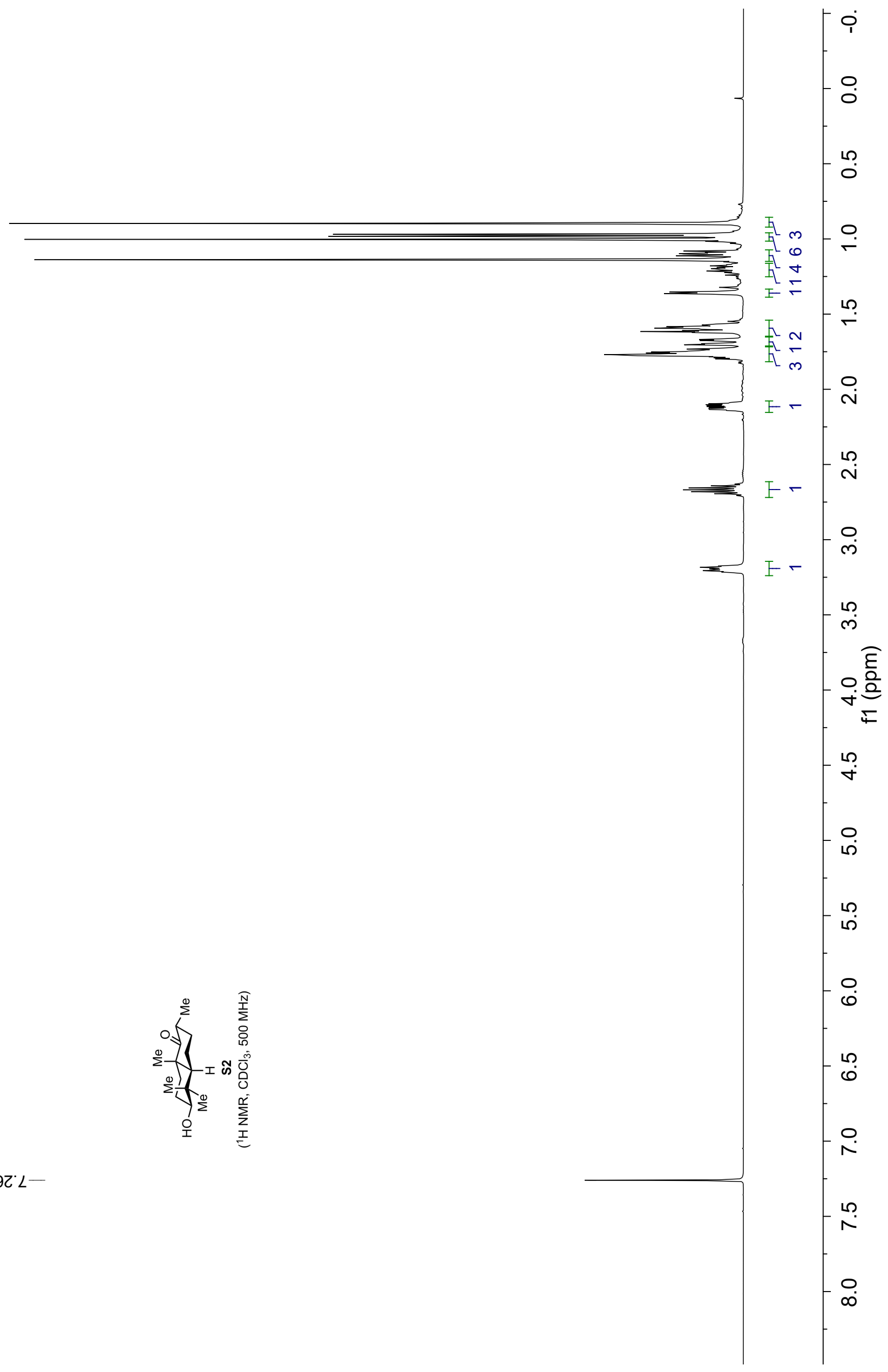




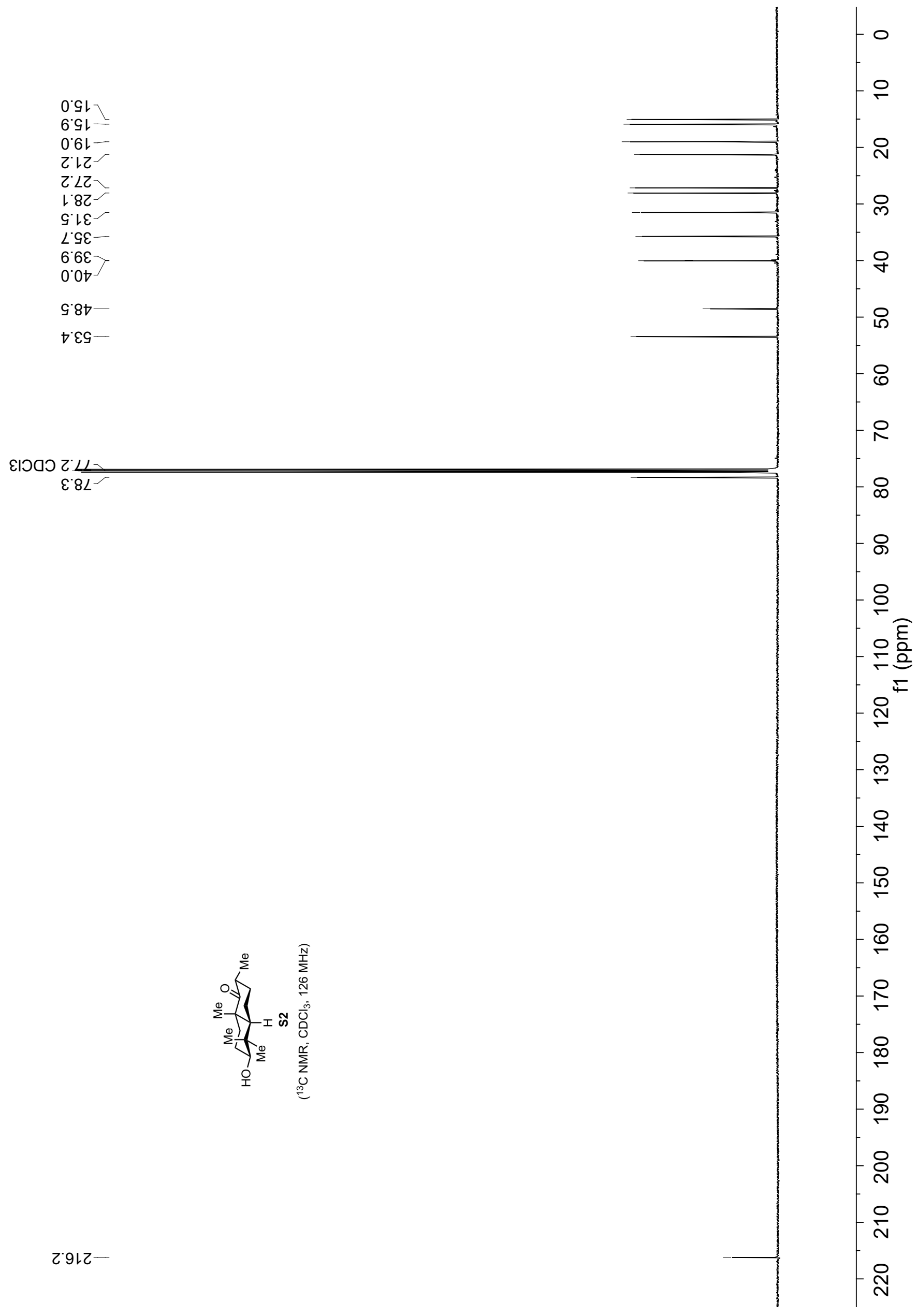




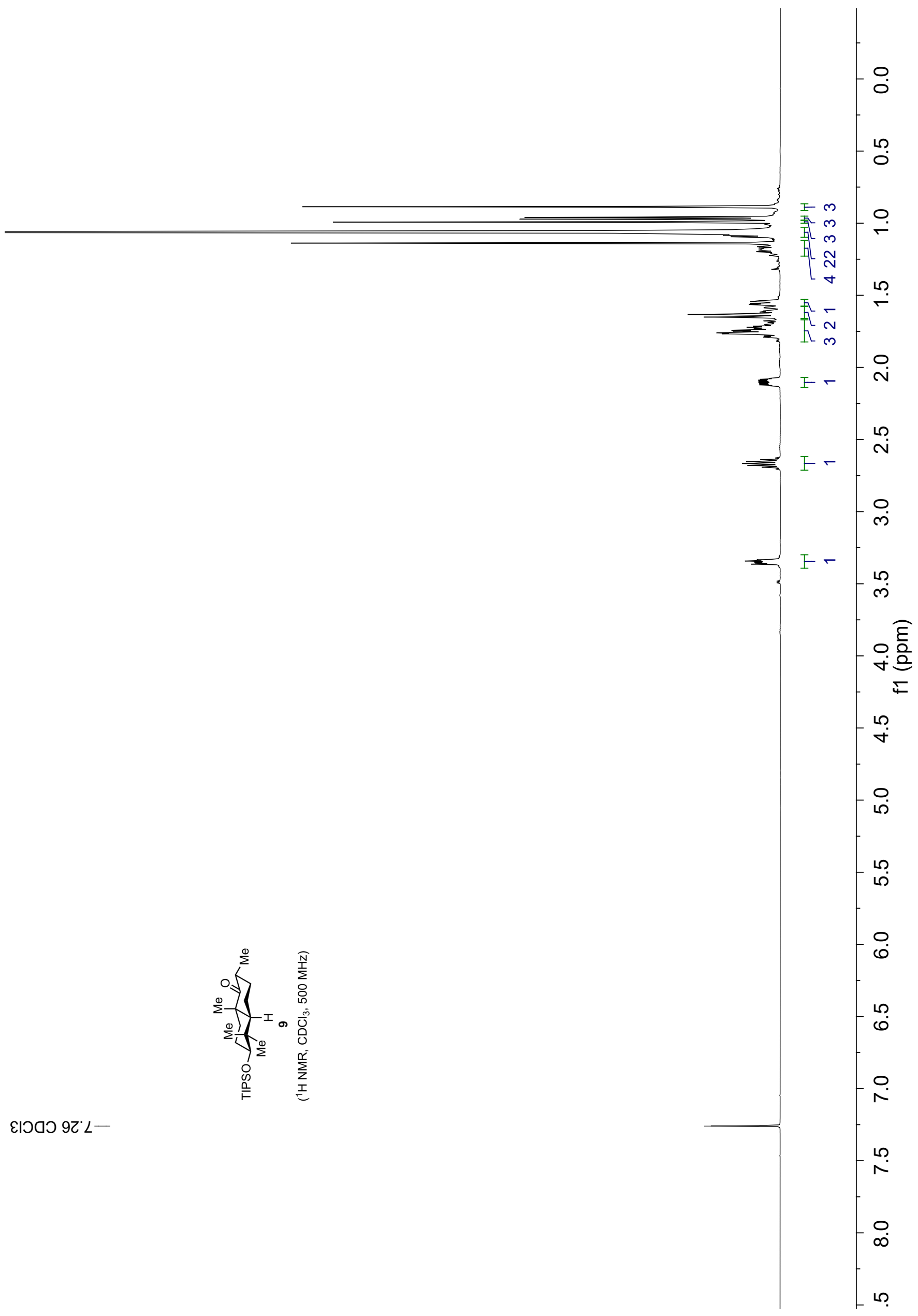




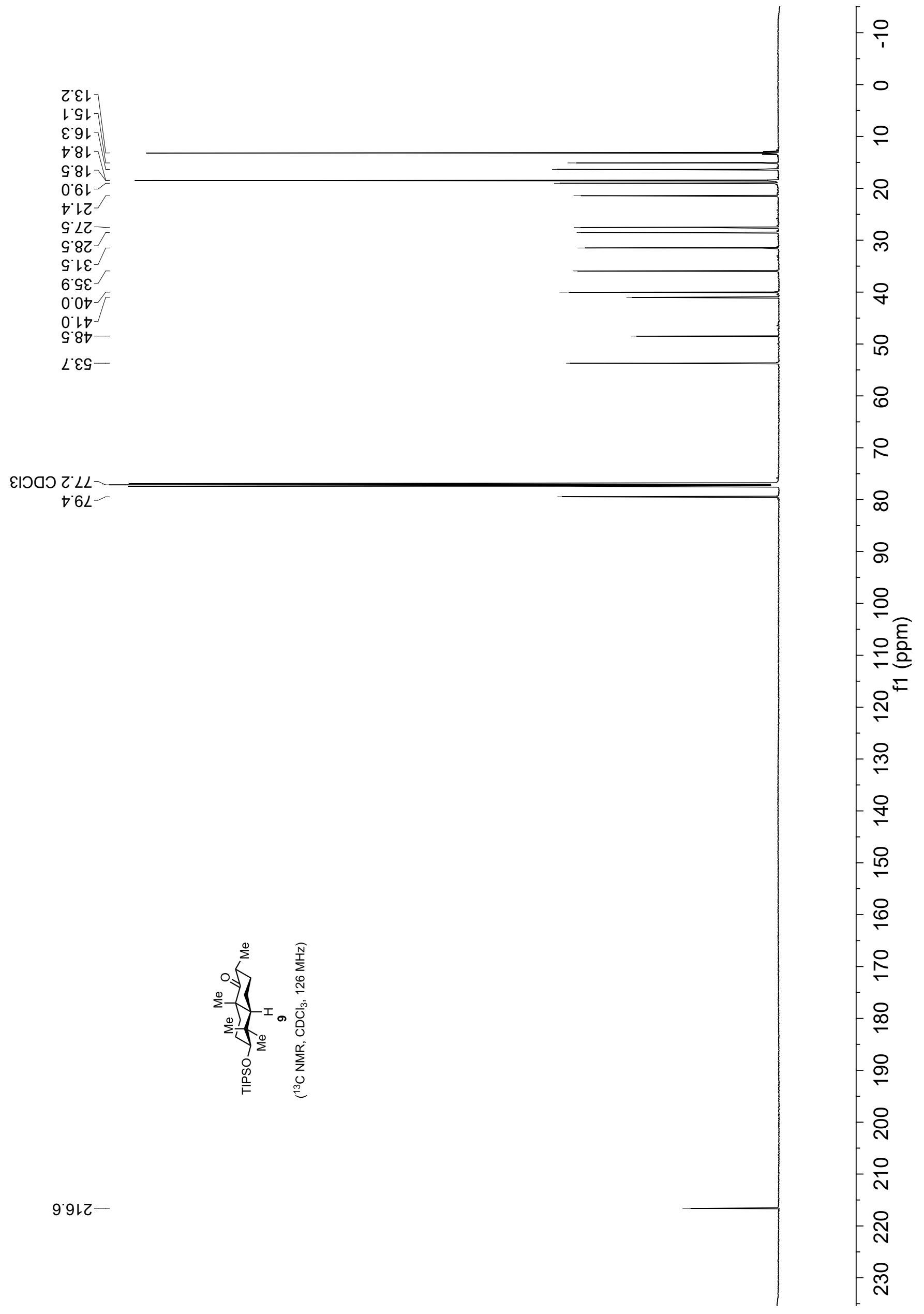




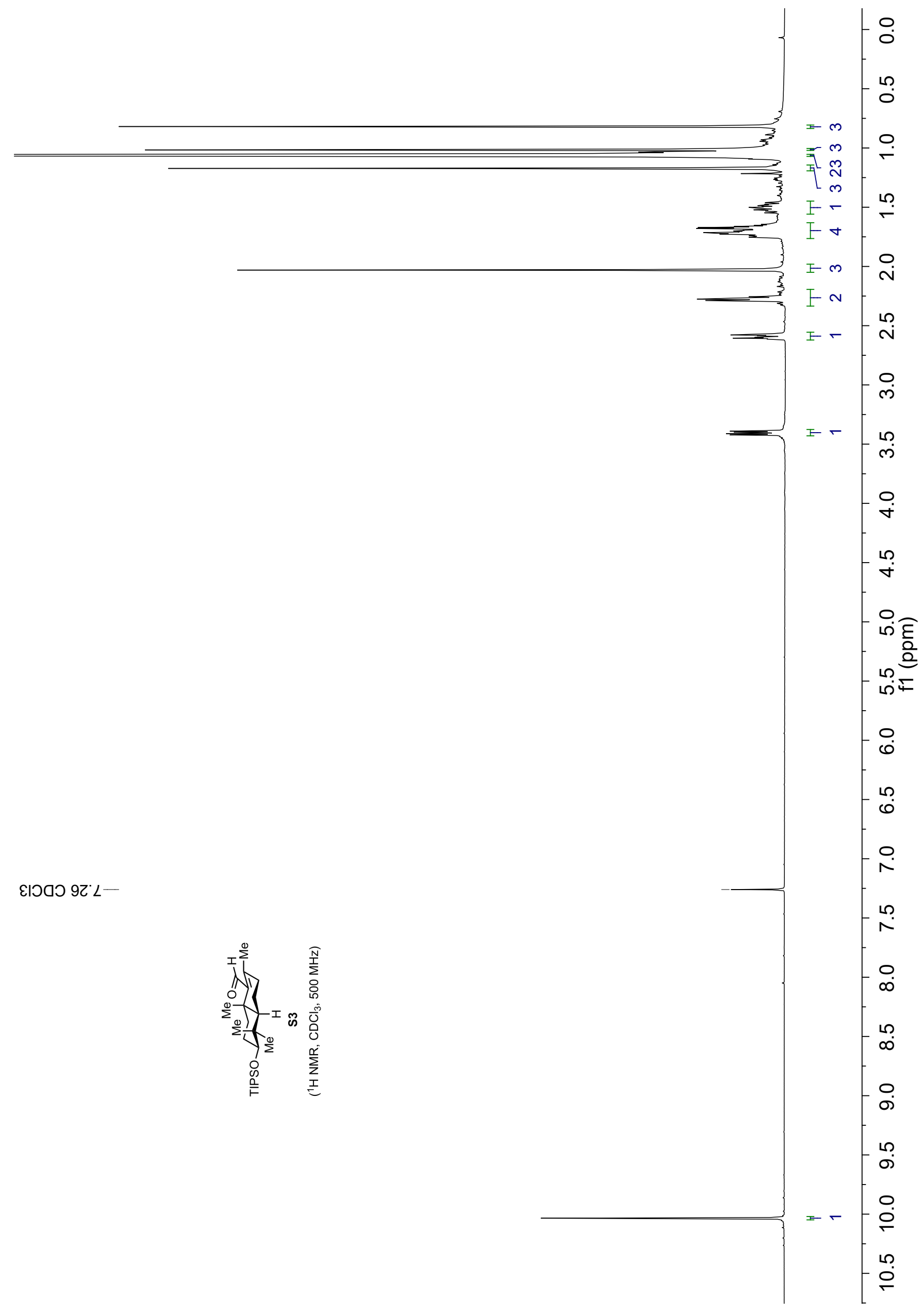


$\tau \cdot \varepsilon \downarrow$

$z \cdot 9 l$

$78 \mathrm{~L}$

$G^{\circ} 8 \mathrm{~L}$

G. 81

l.6l-

$\mathrm{z} 0 \mathrm{z}$

$\varepsilon .8 Z$

8.82

$G^{\prime} \nabla \varepsilon$

$0 \angle \varepsilon$

$\nabla^{\circ} \angle \varepsilon$

$20 \nabla^{-}$

E'L-

ยเวดว 乙' LL$66 L$

૬`๕ャレー

๑๋๑レー

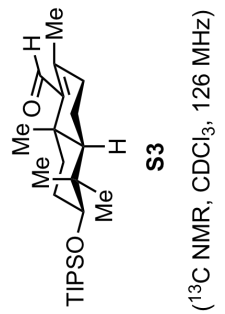

t゙ZL- 


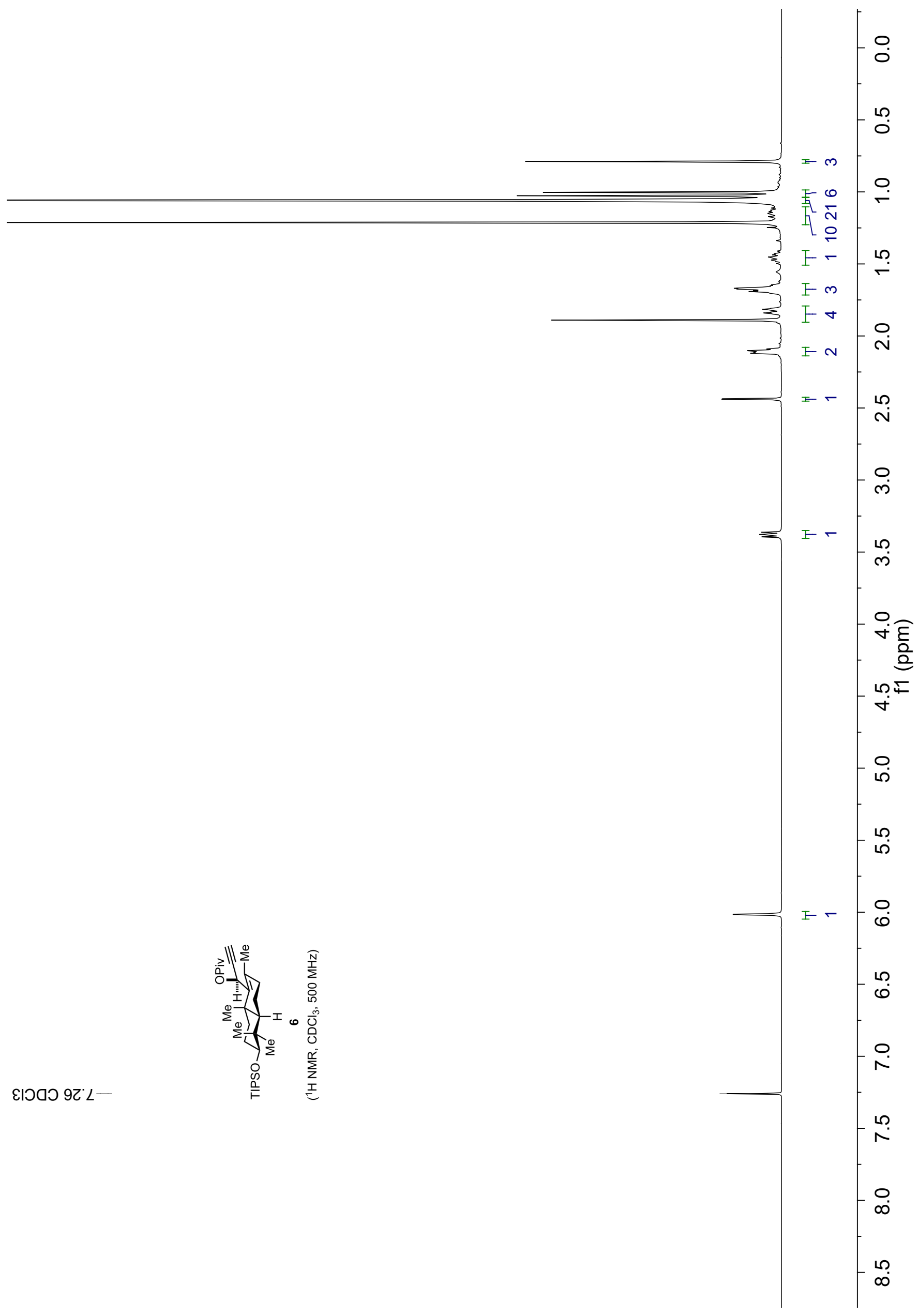




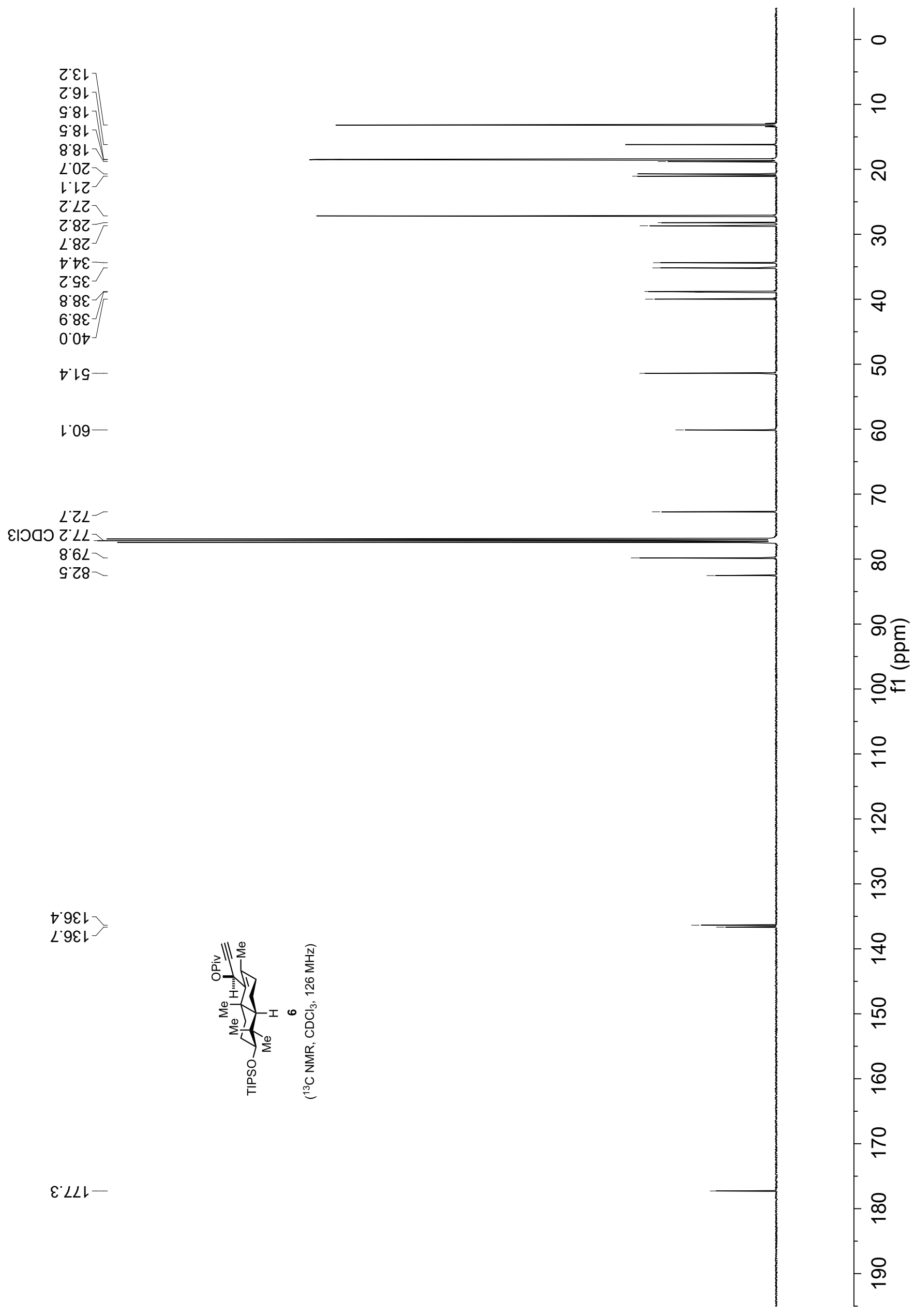




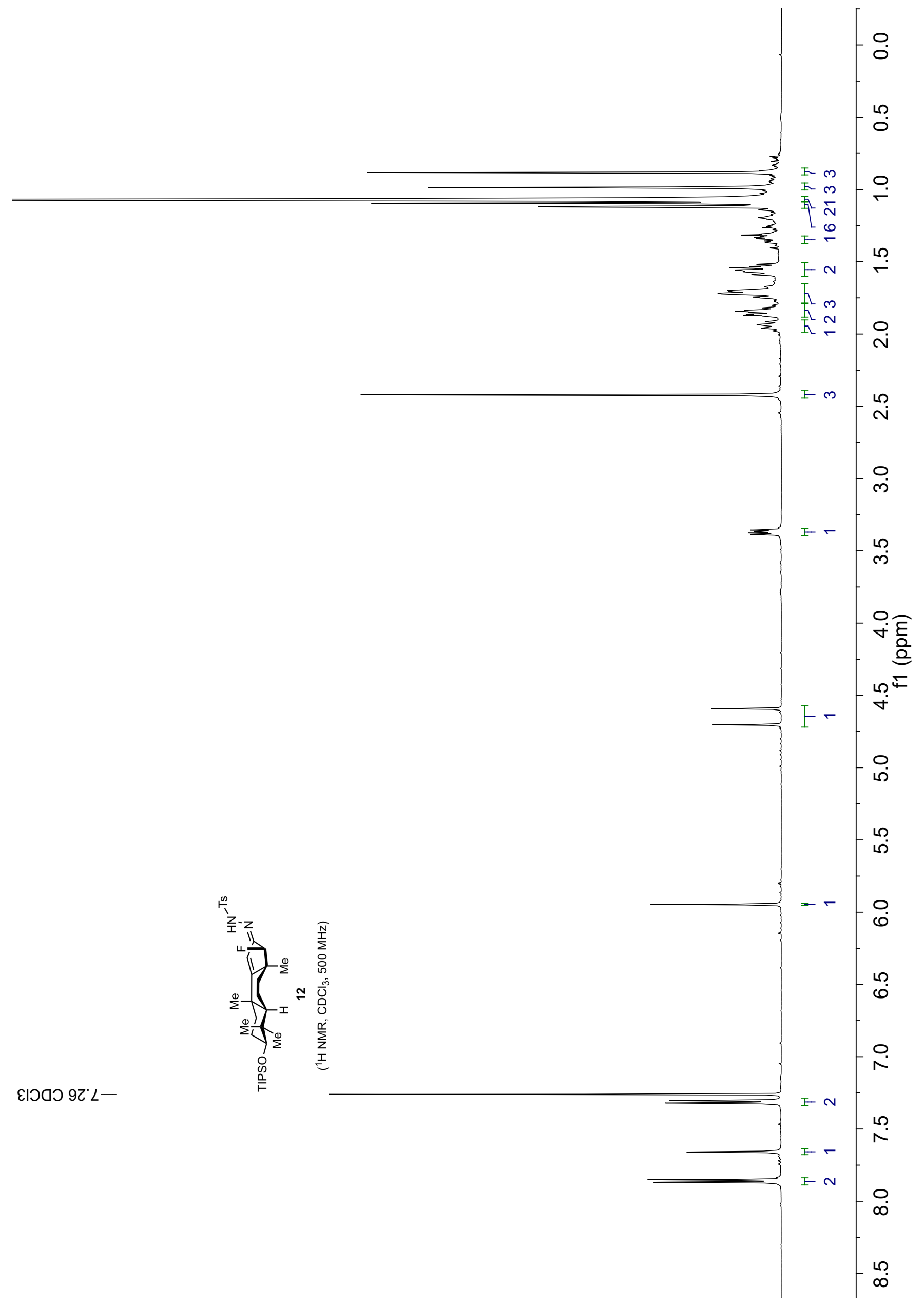




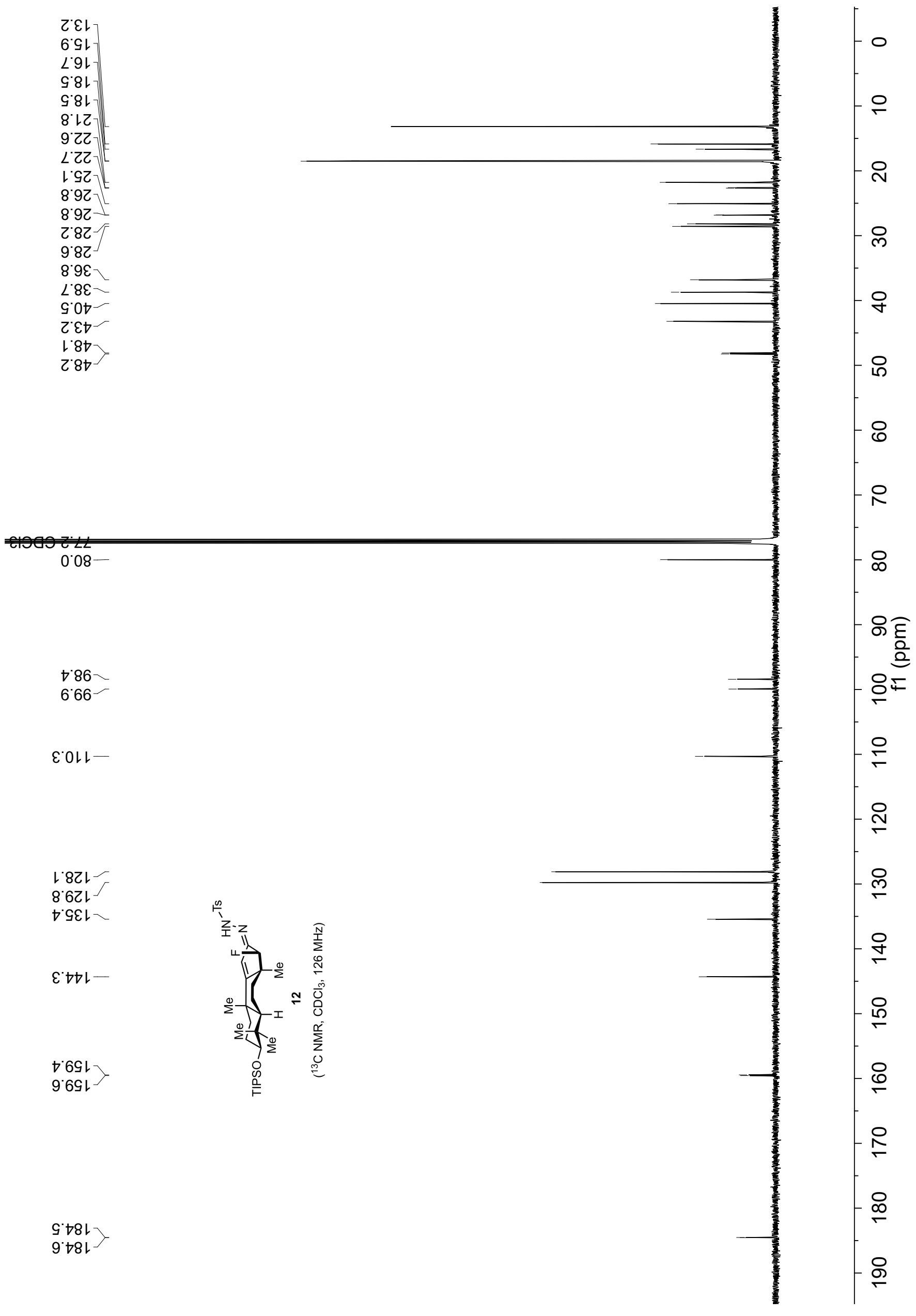




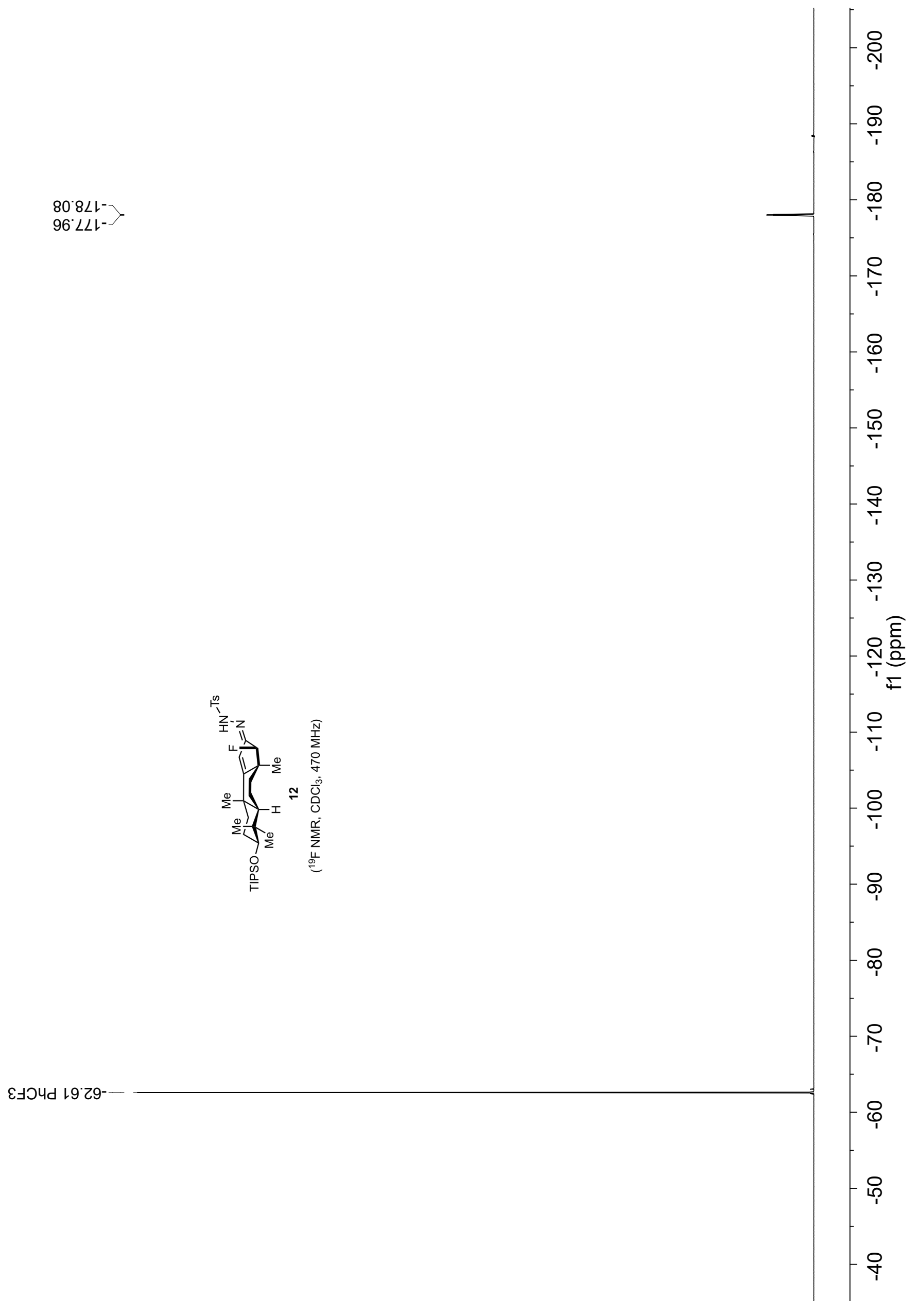




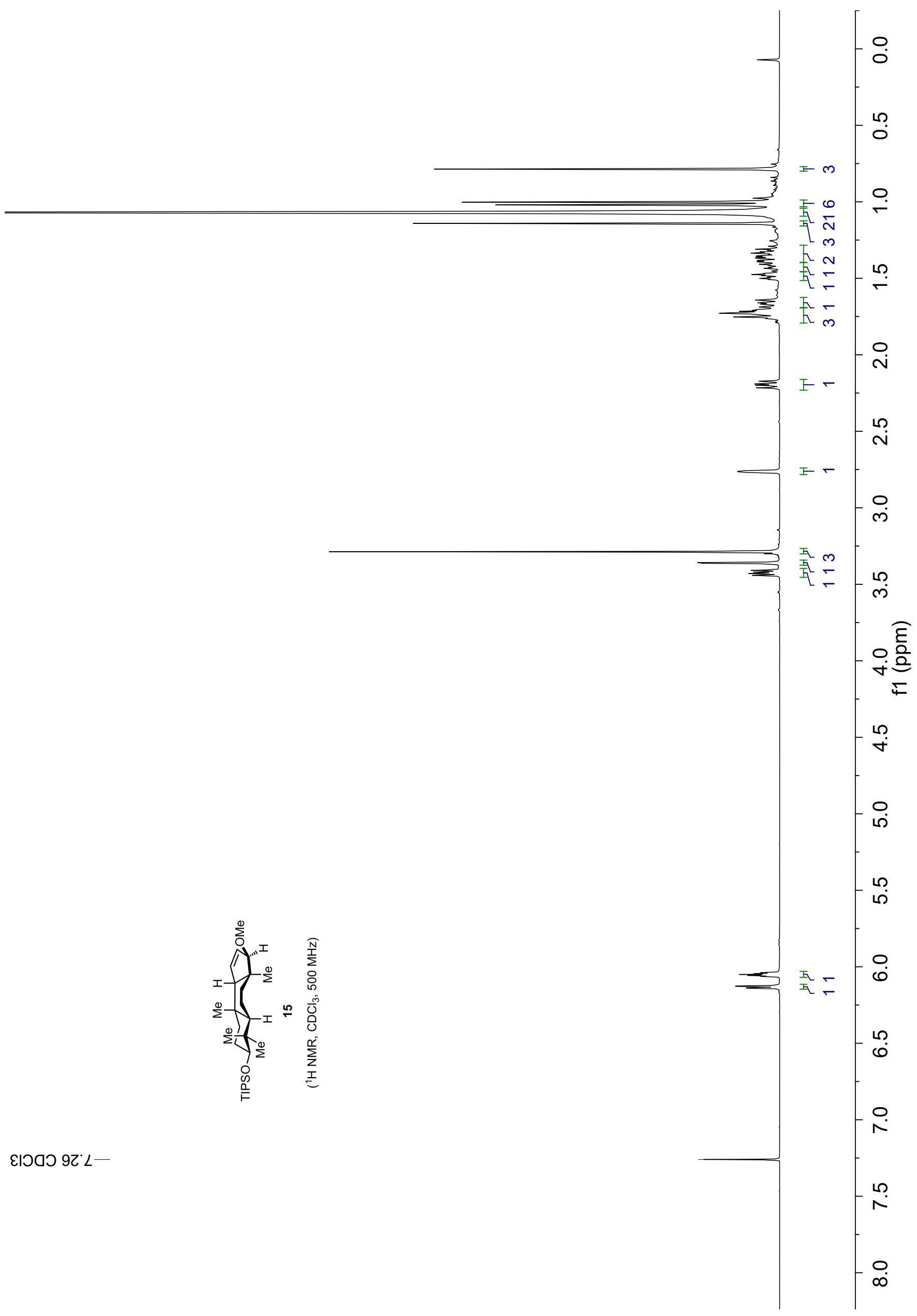




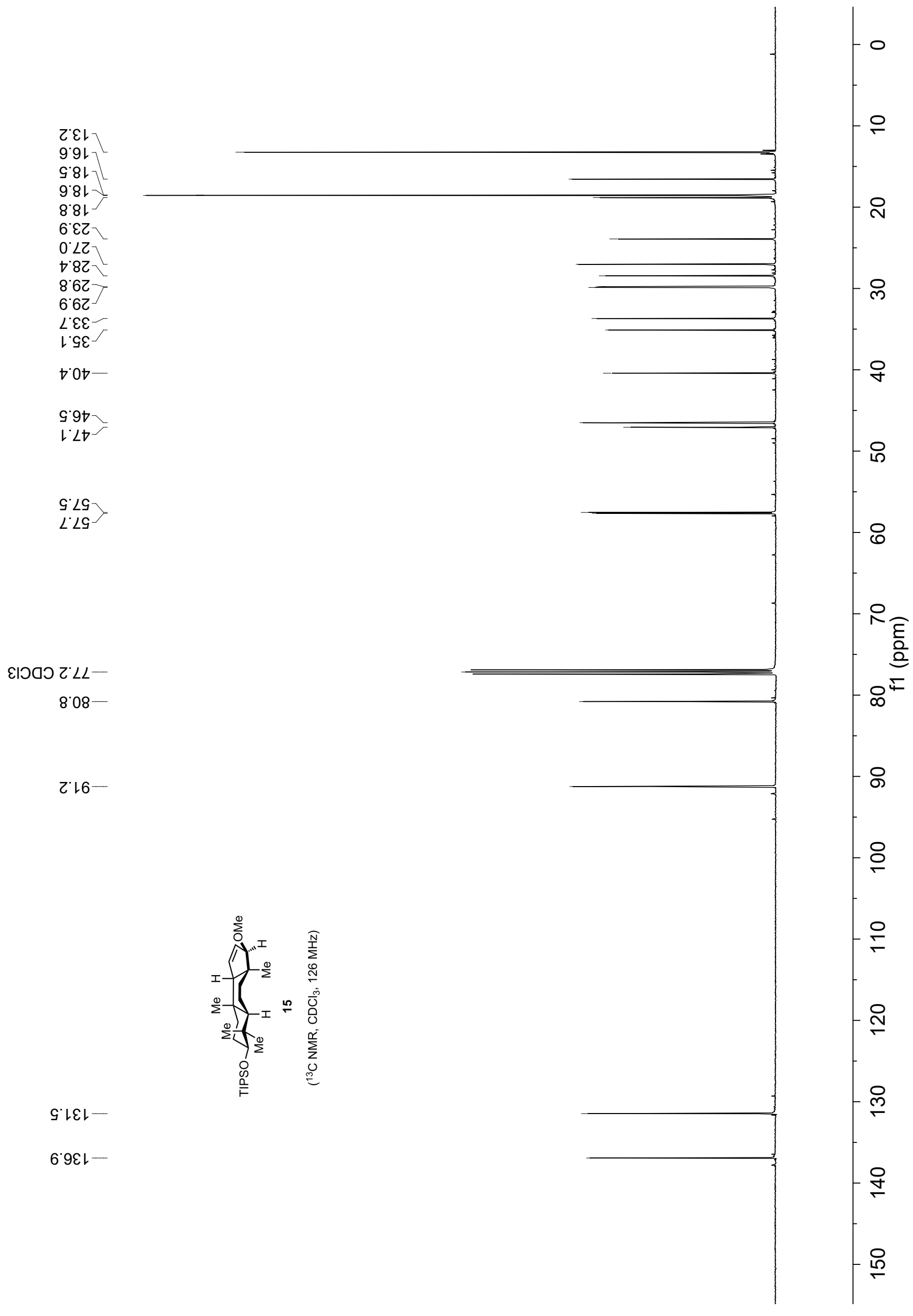


(mdd) H

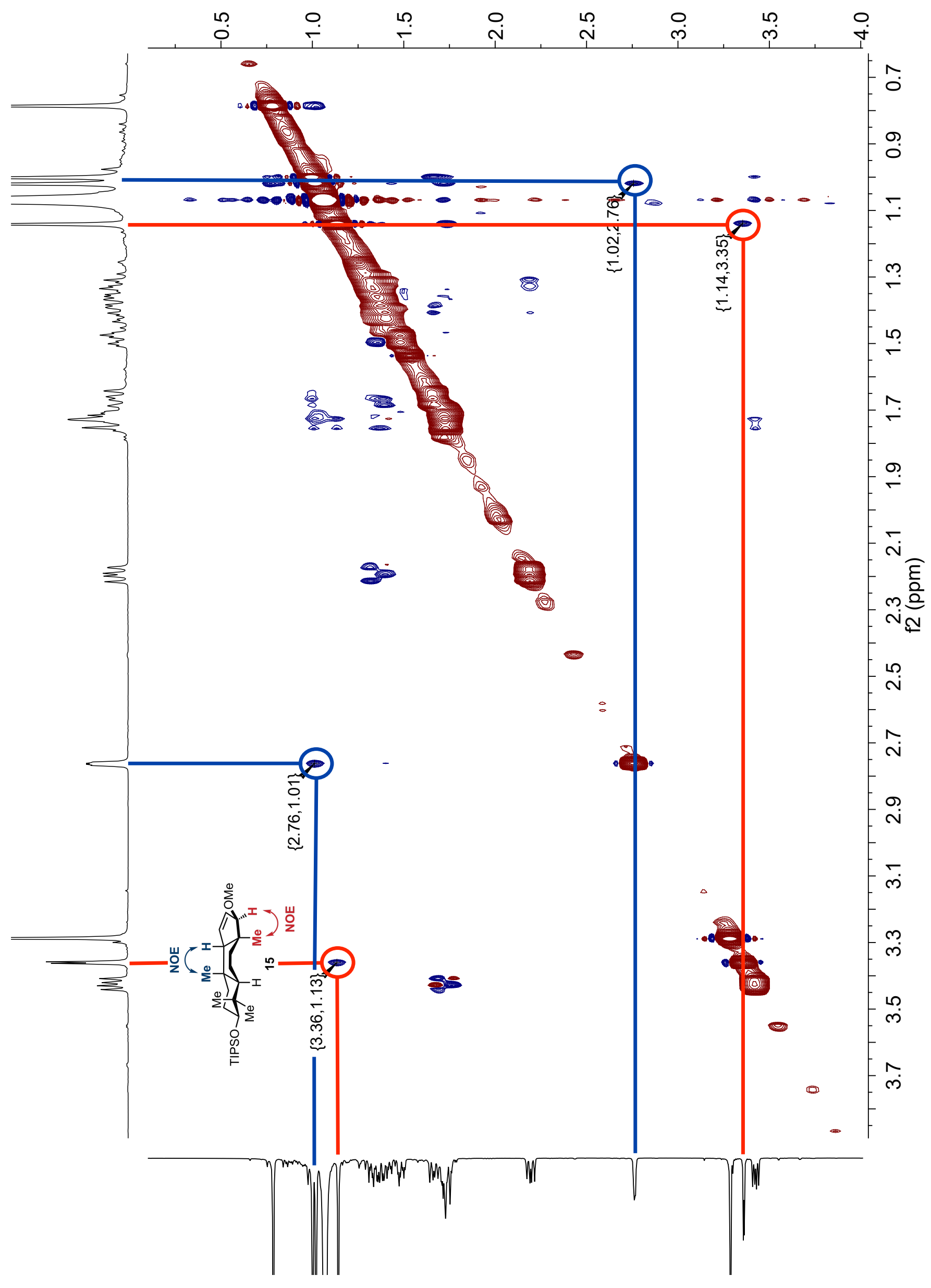




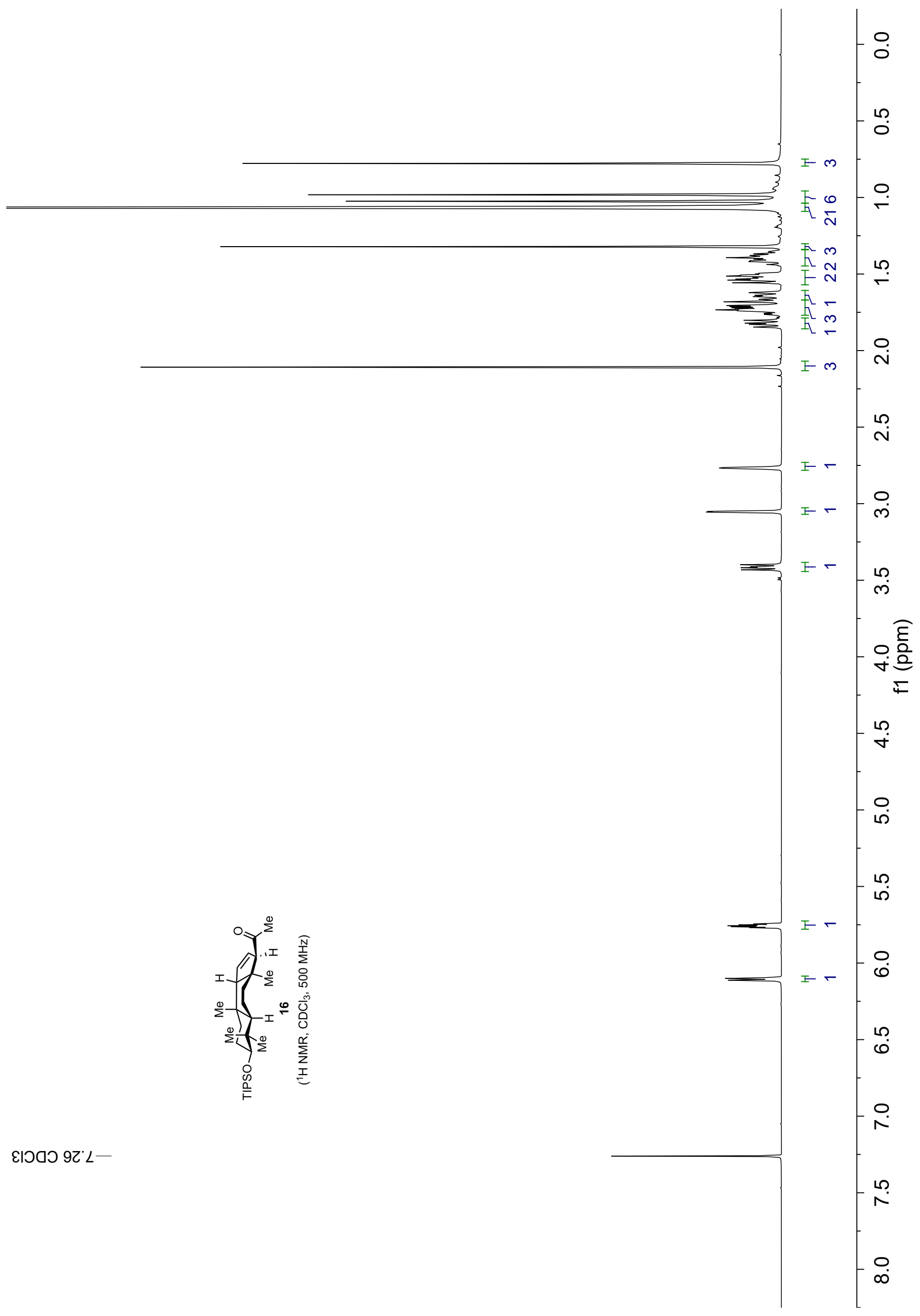




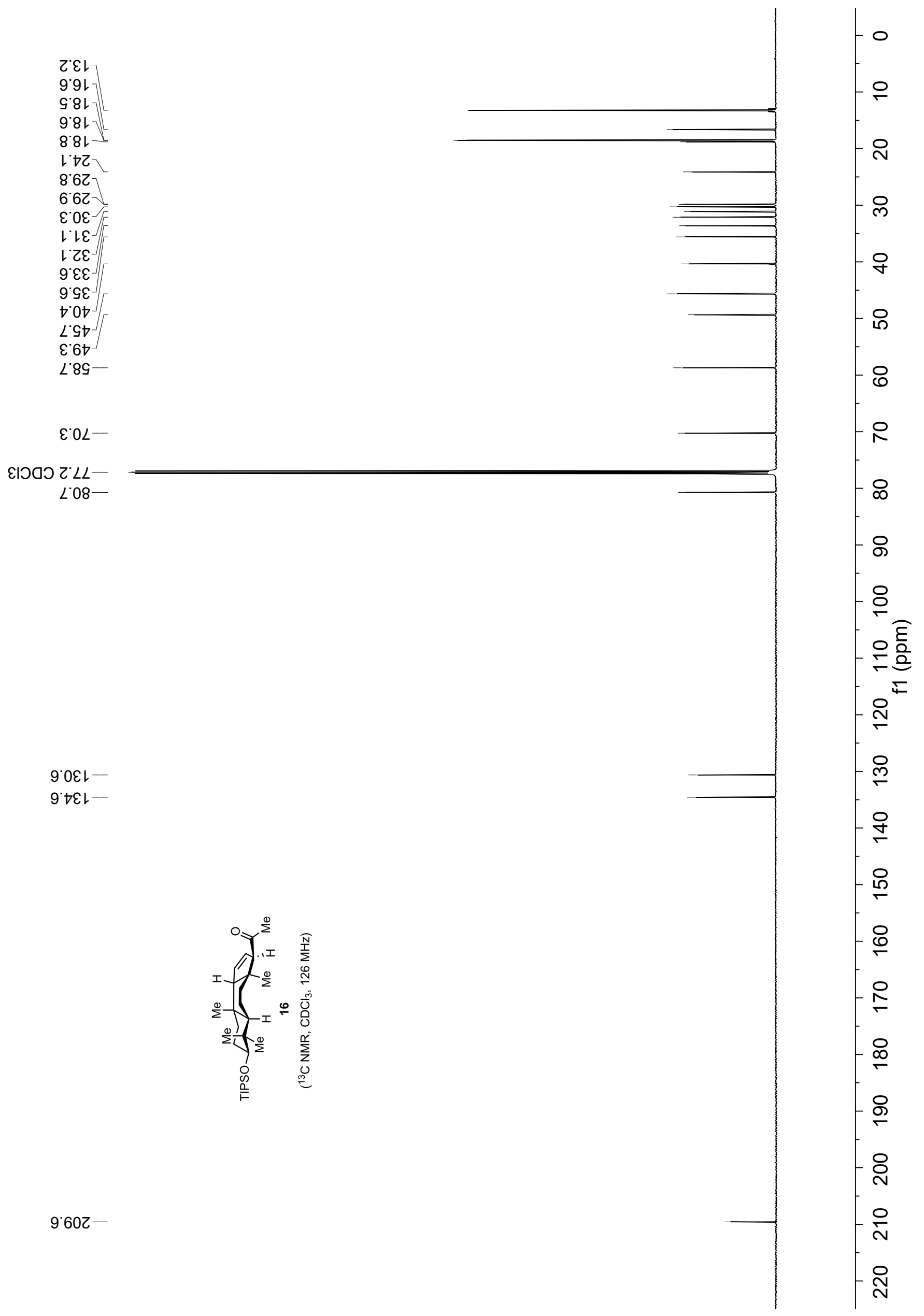


(mdd) H

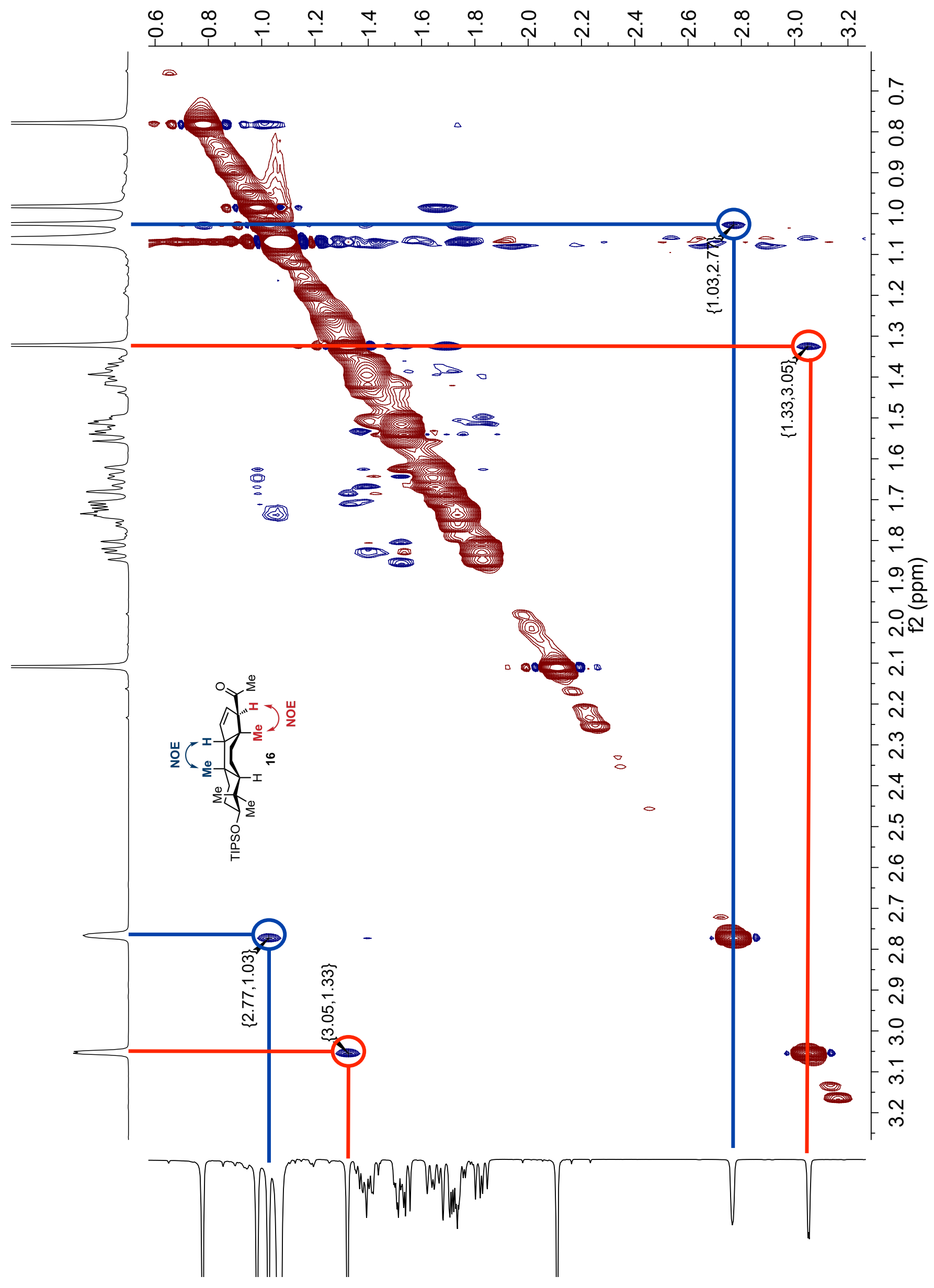




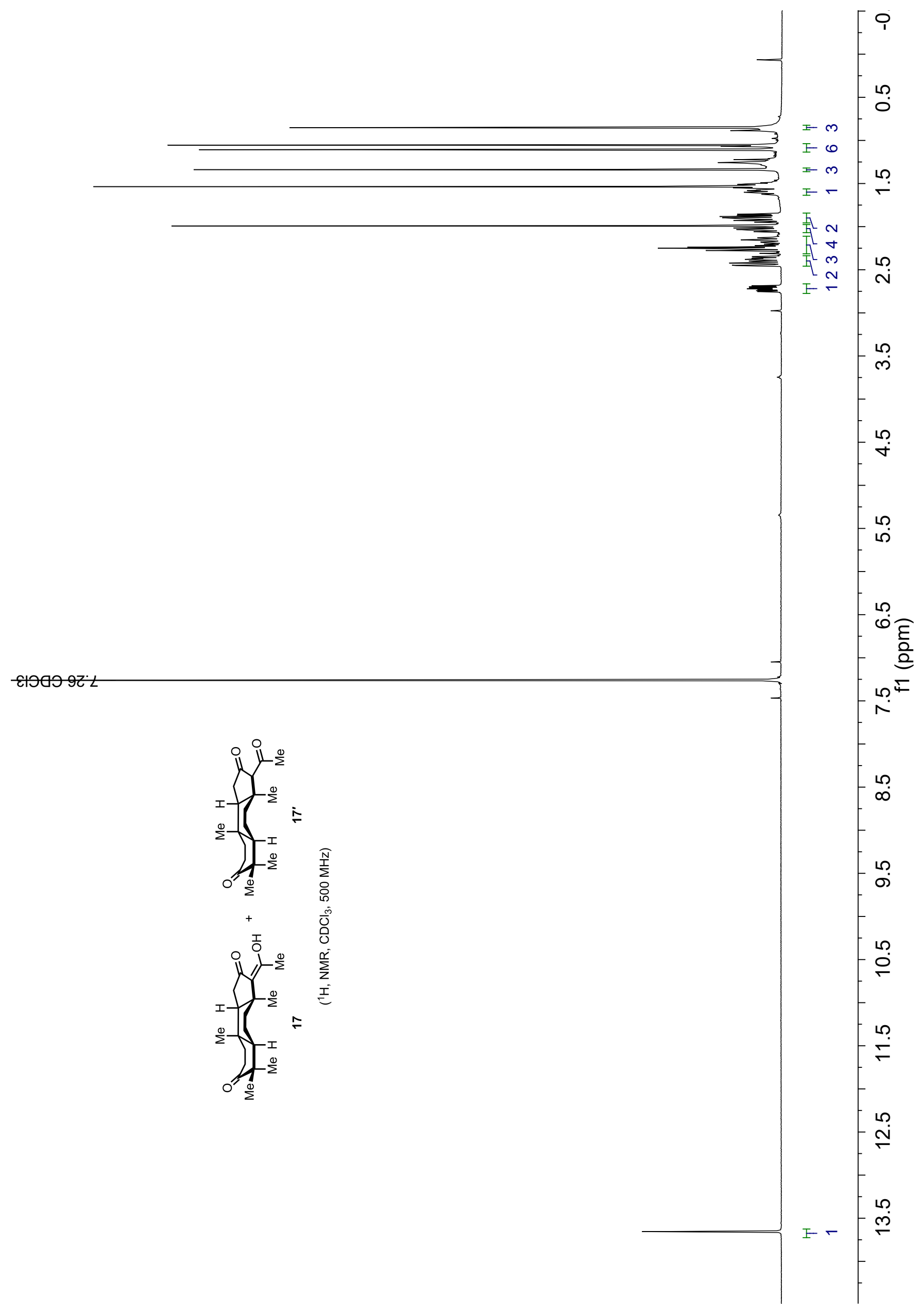




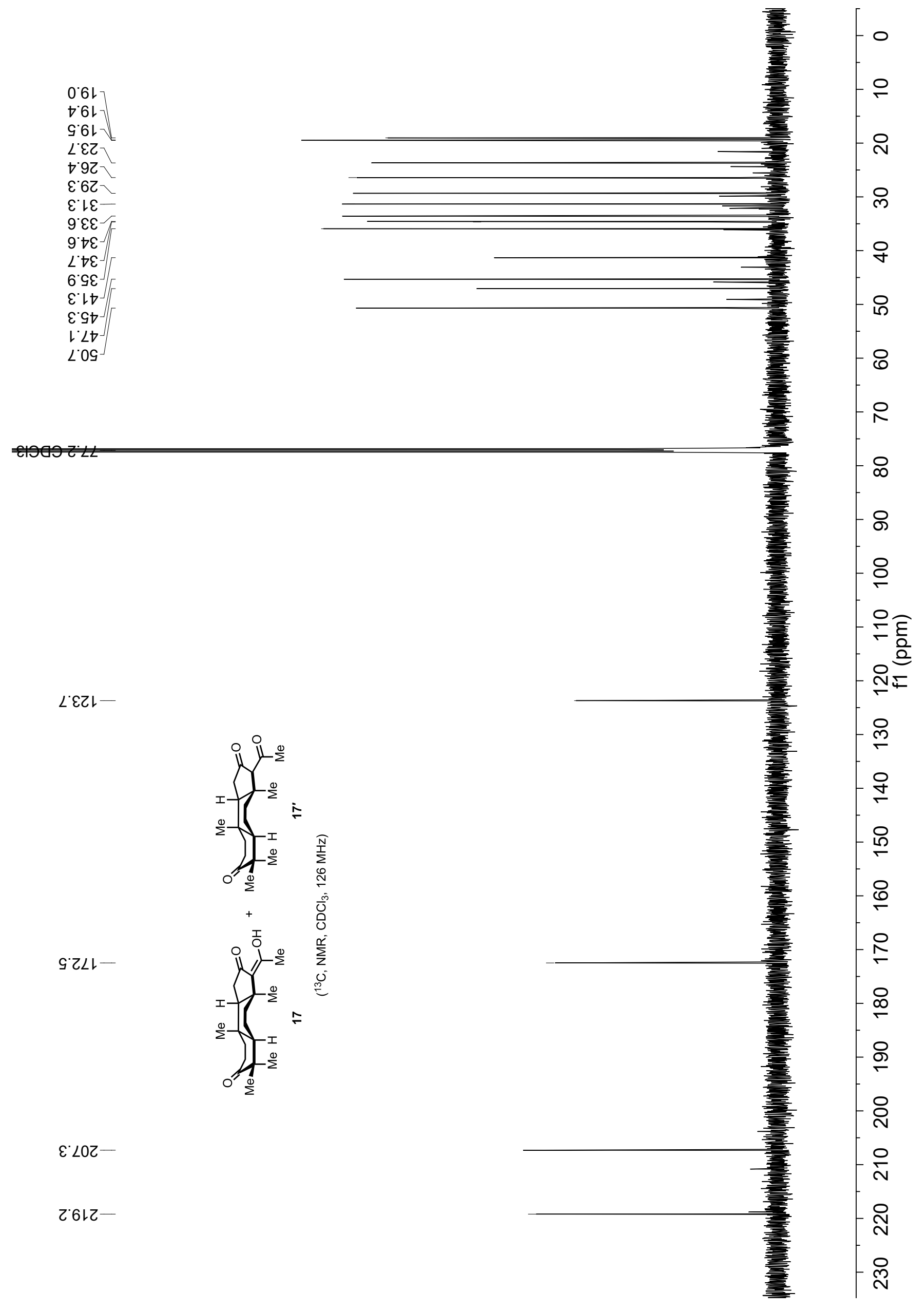




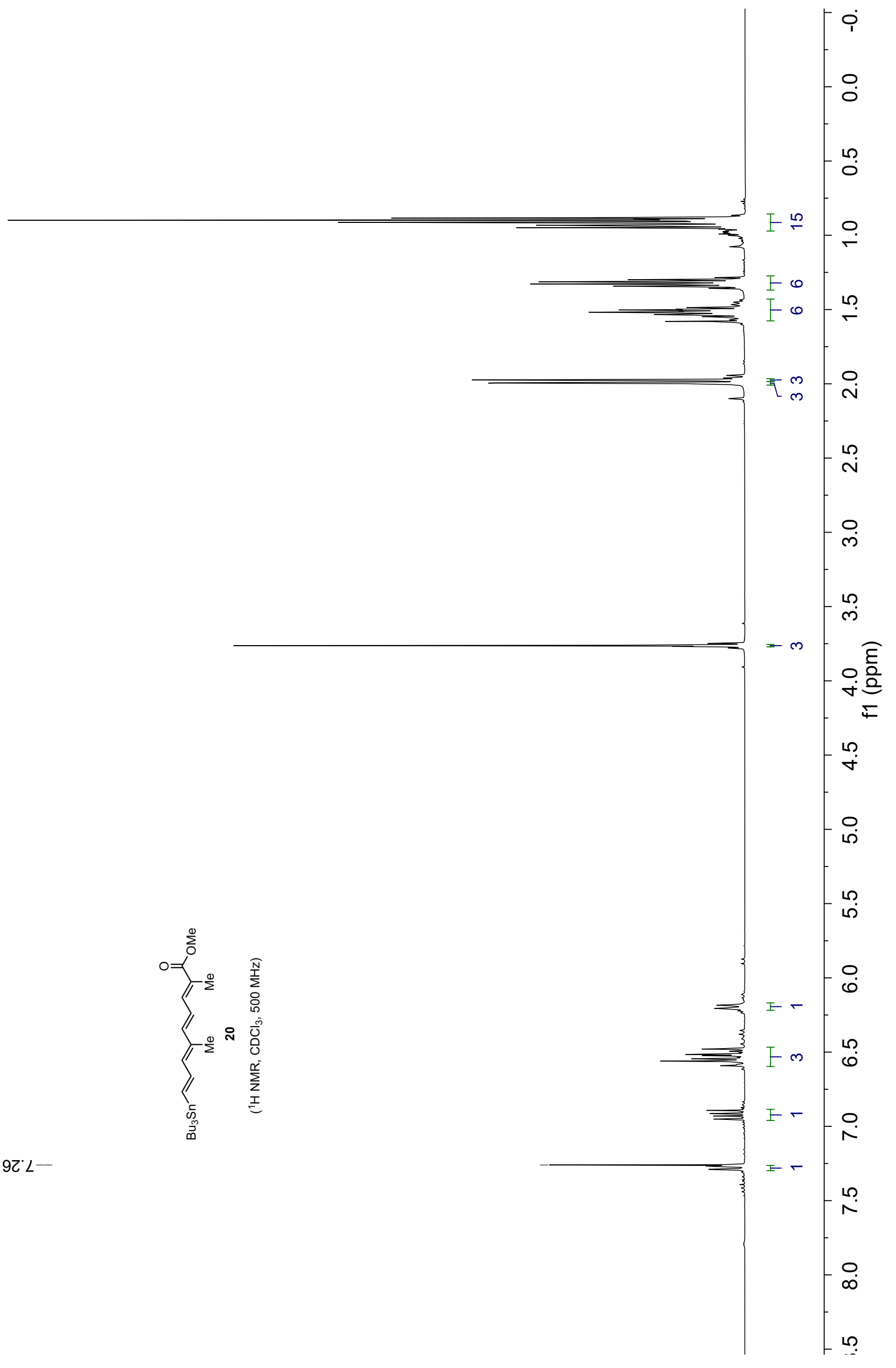




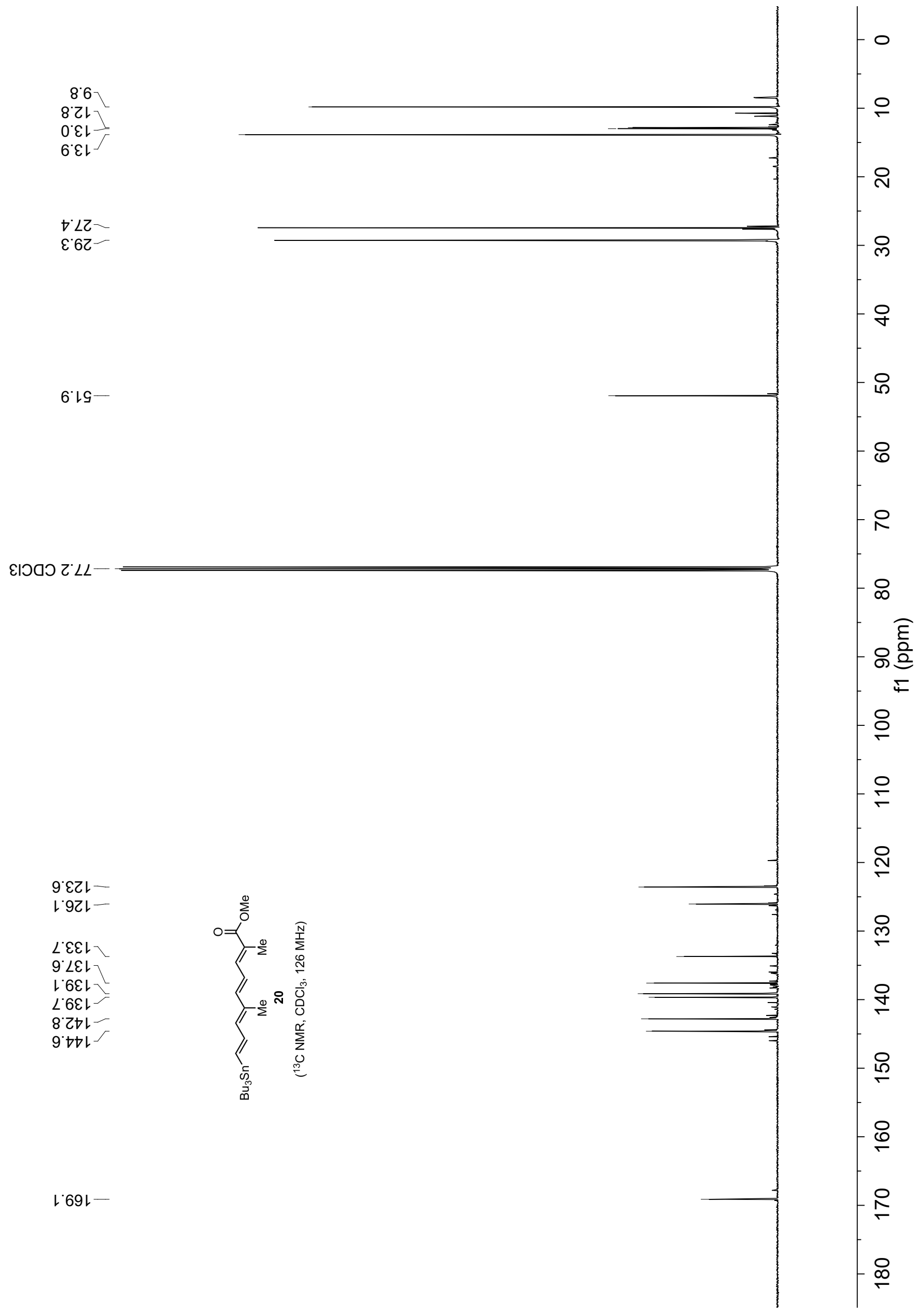




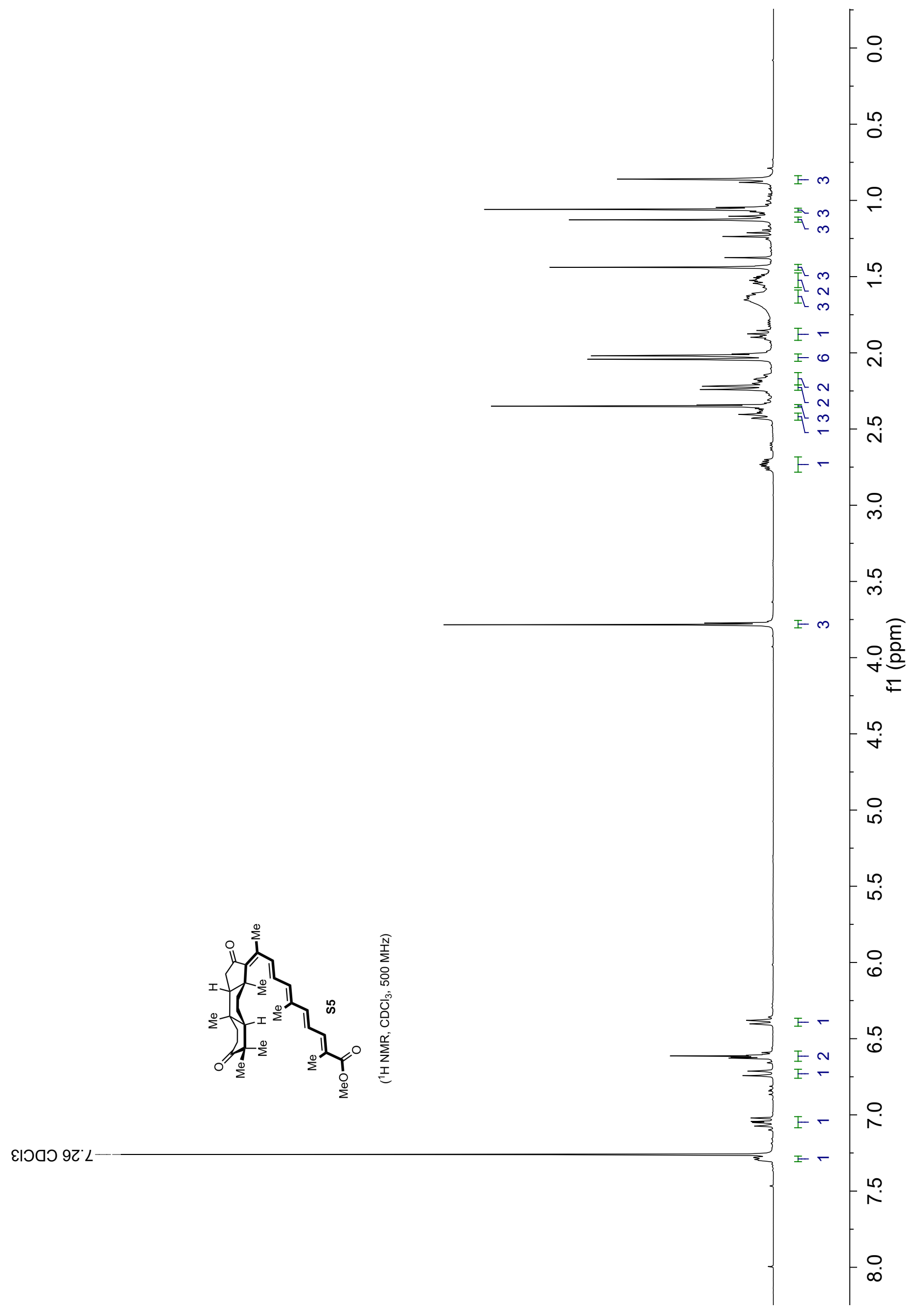




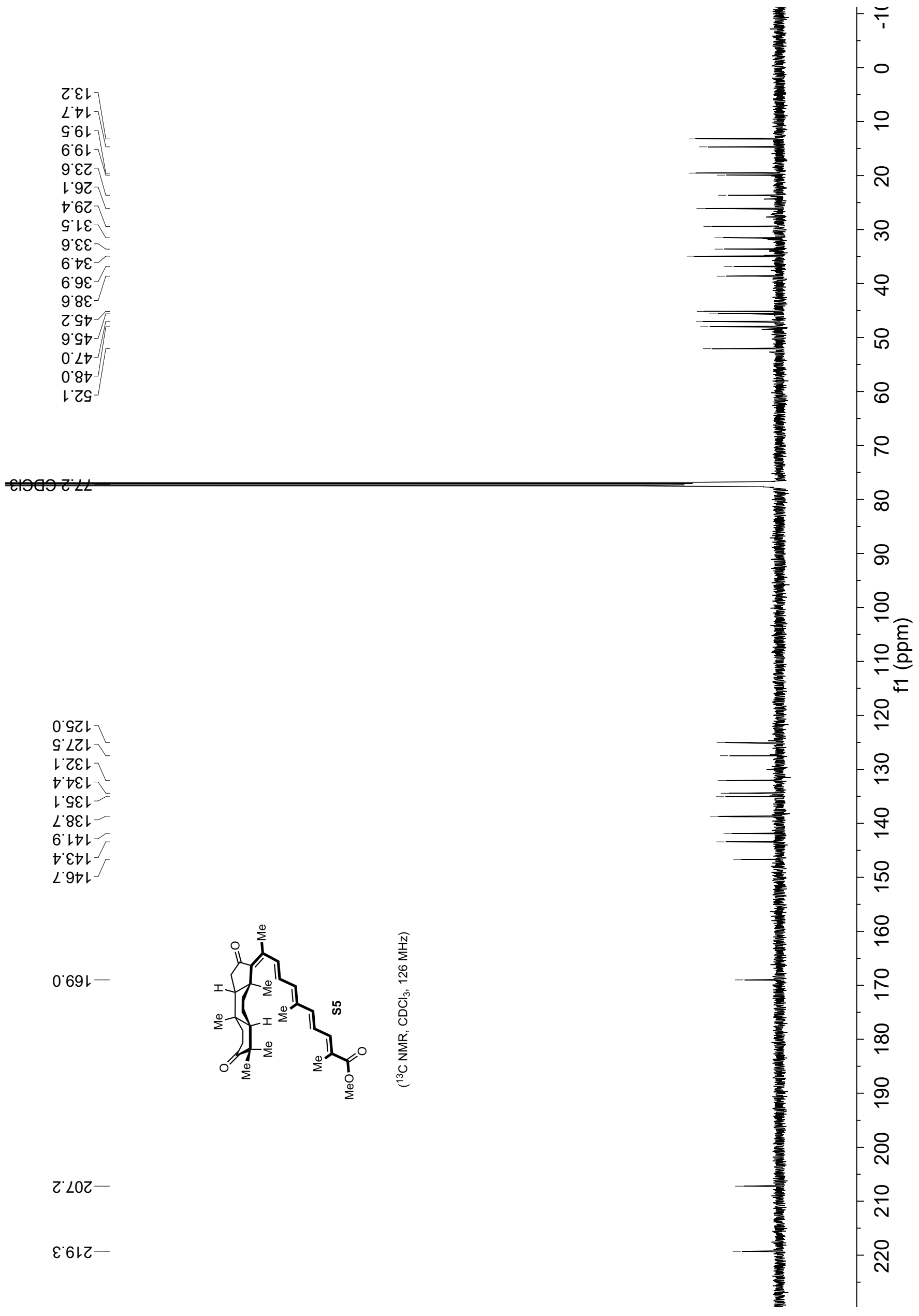




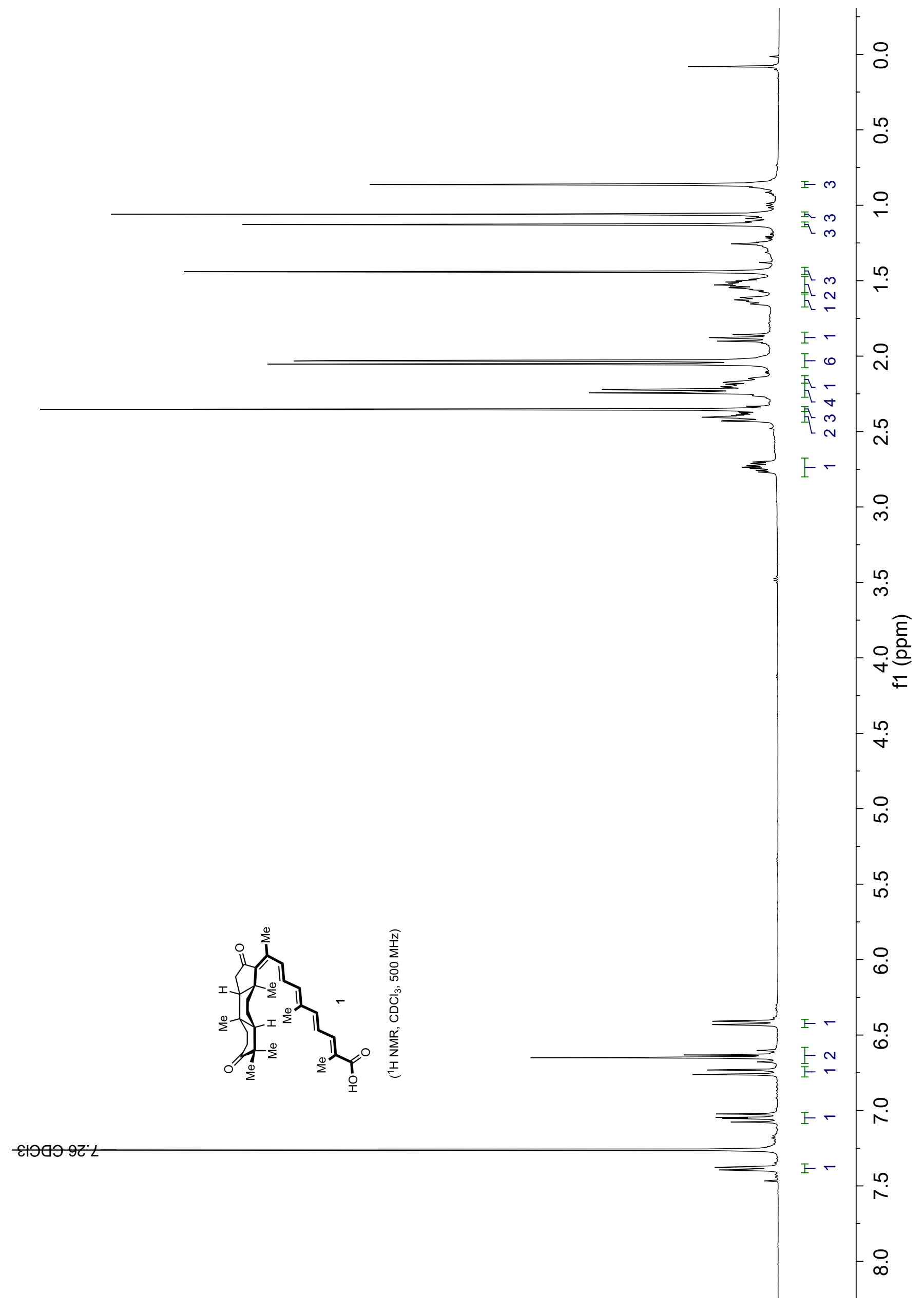




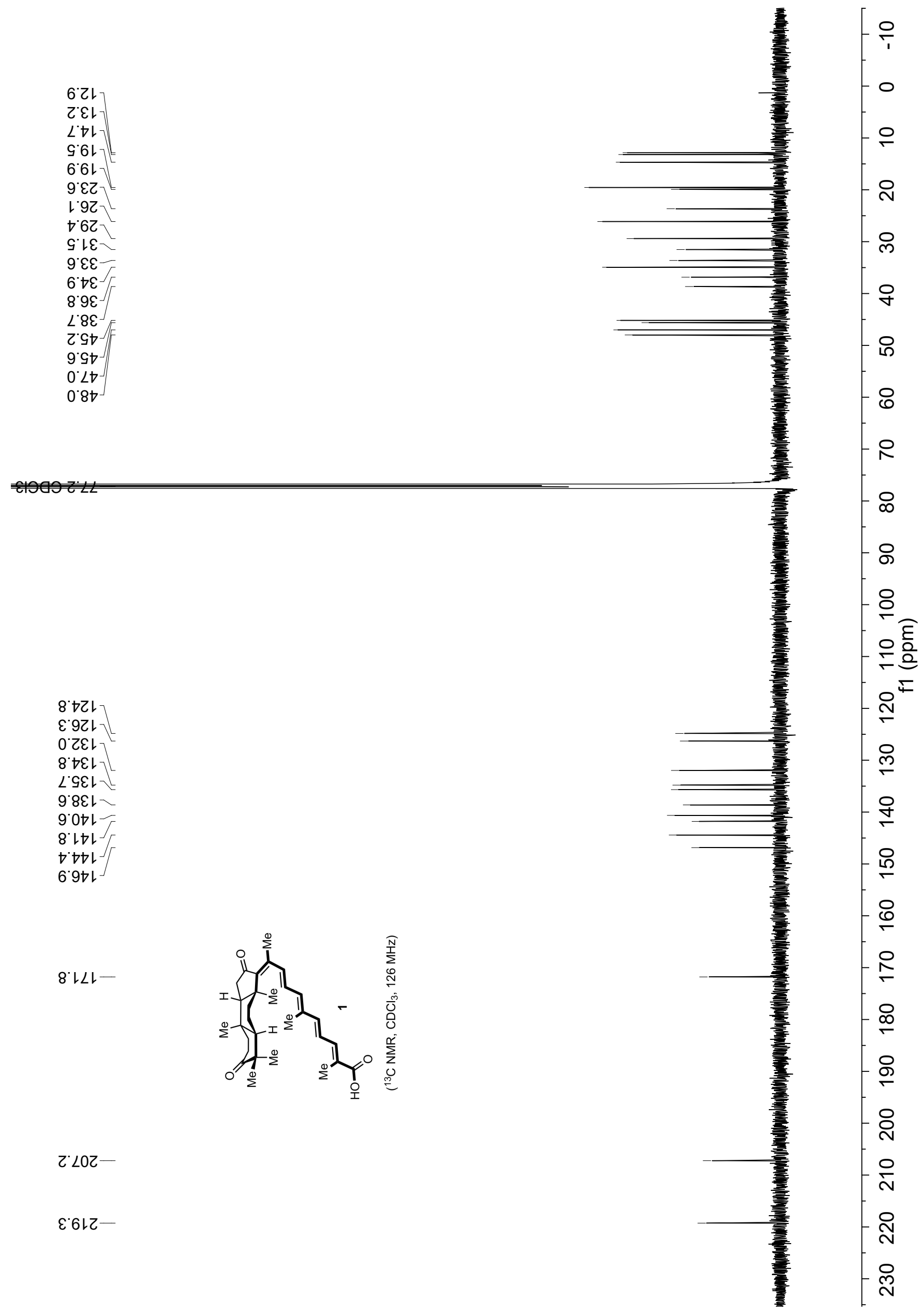


(mdd) If
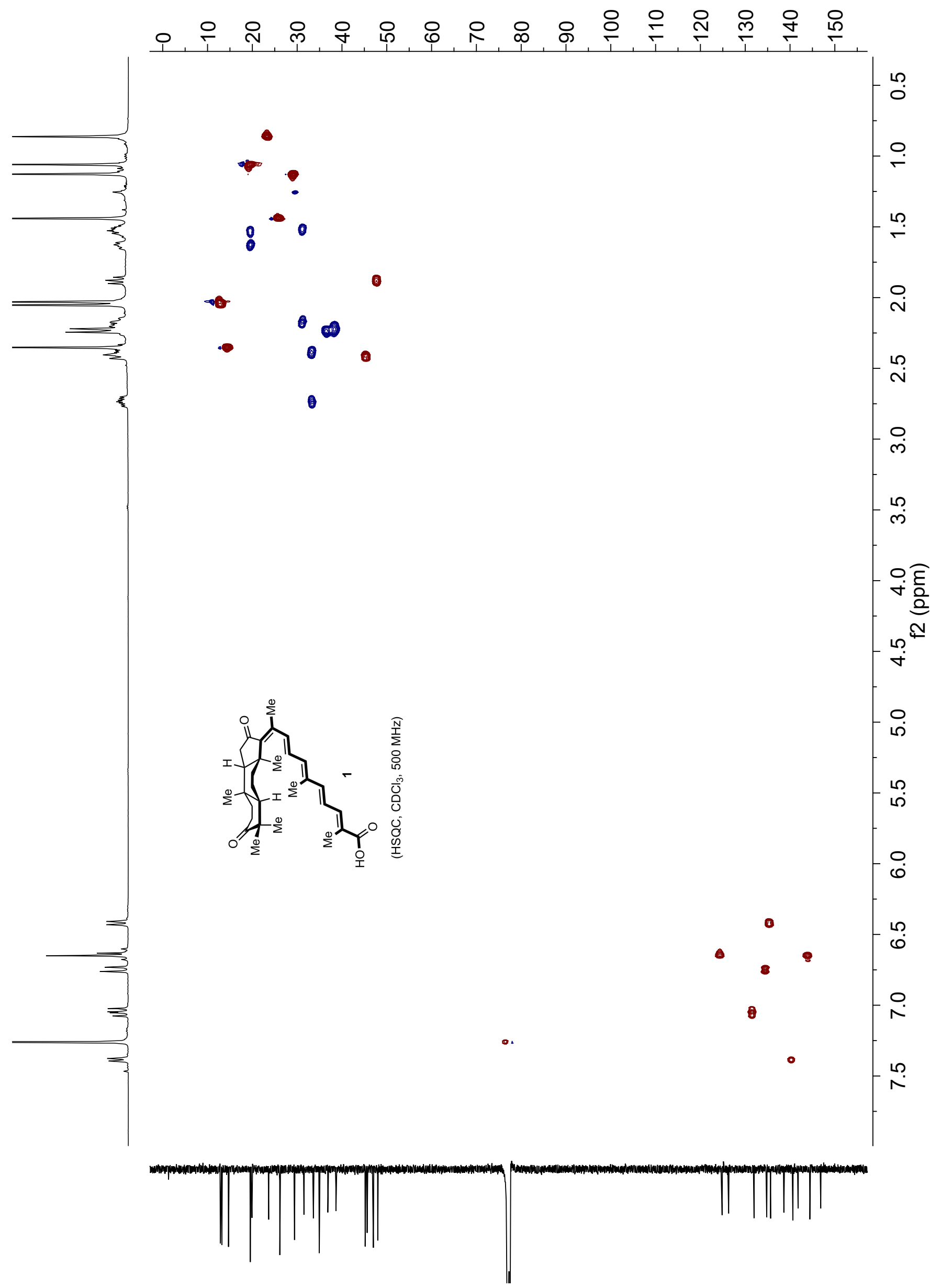
(mdd) if
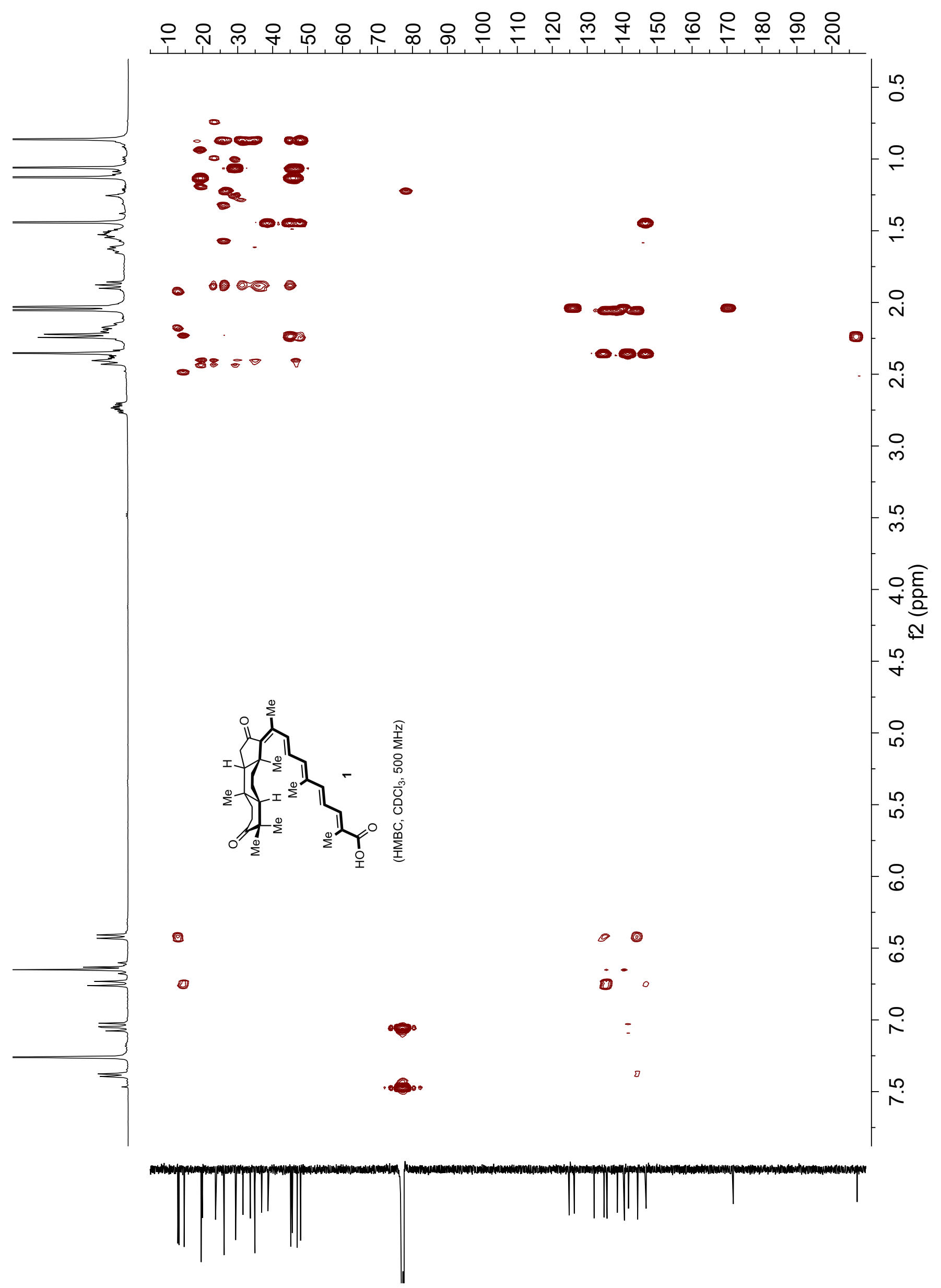


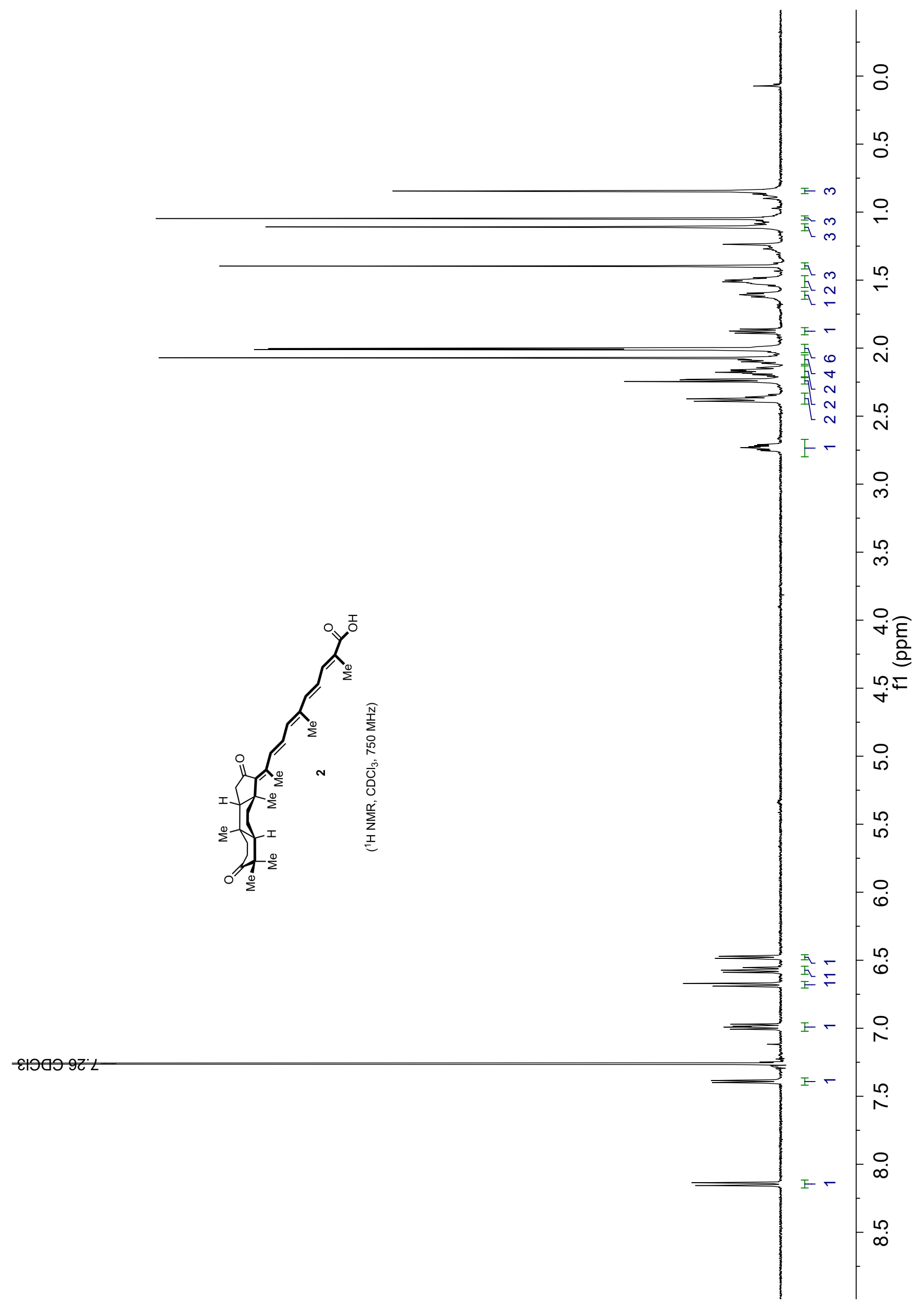




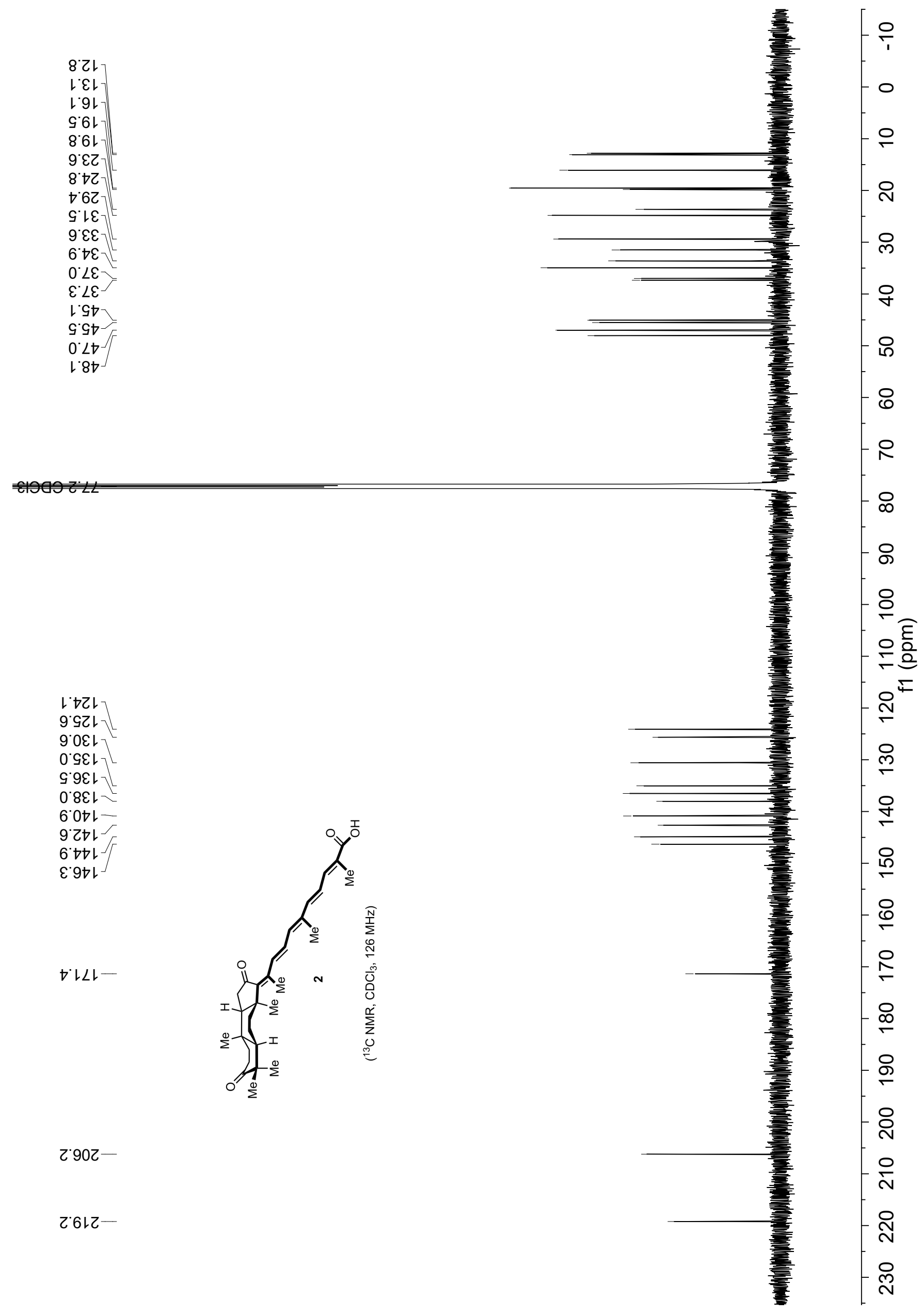




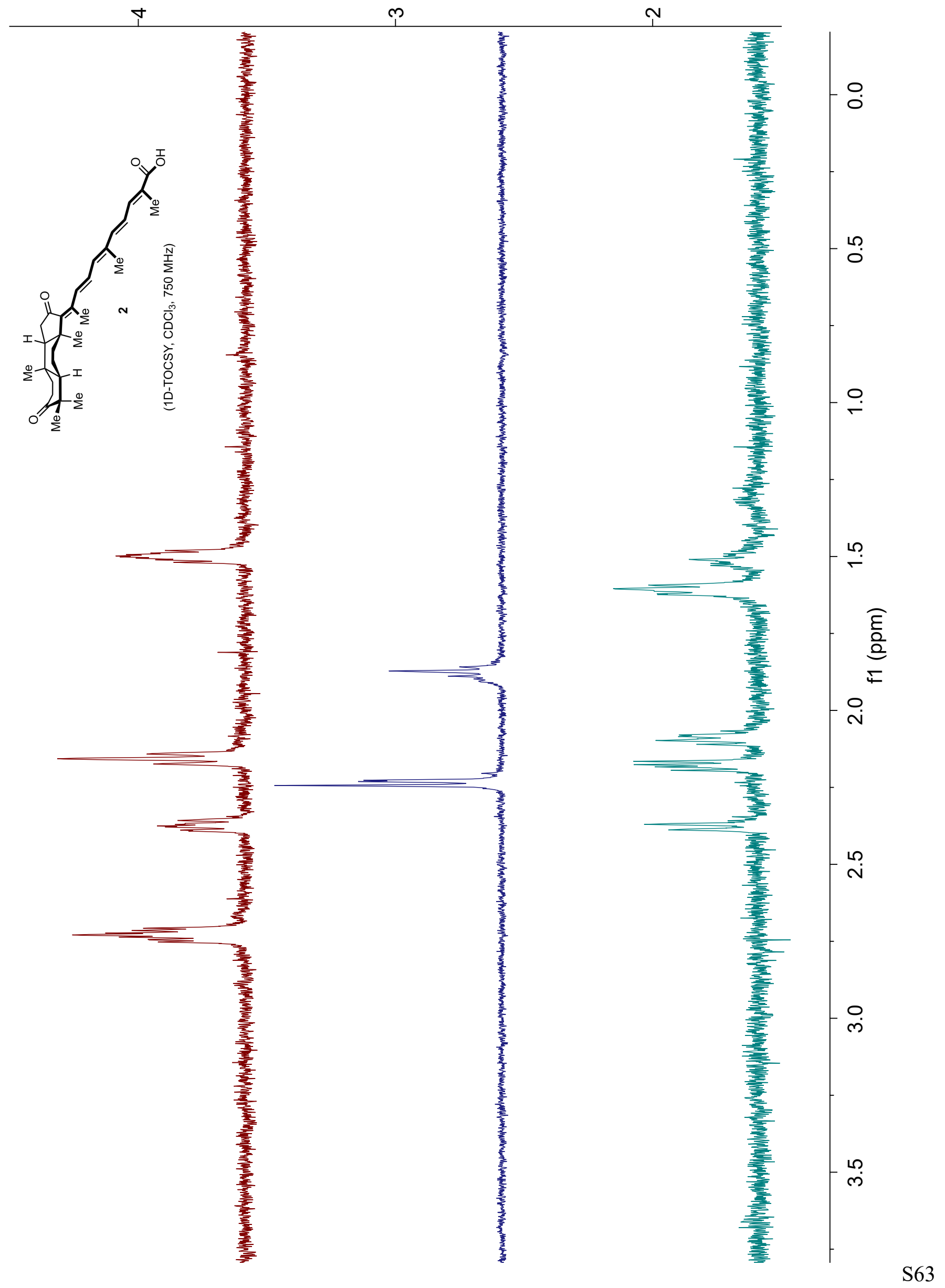


(mdd) If

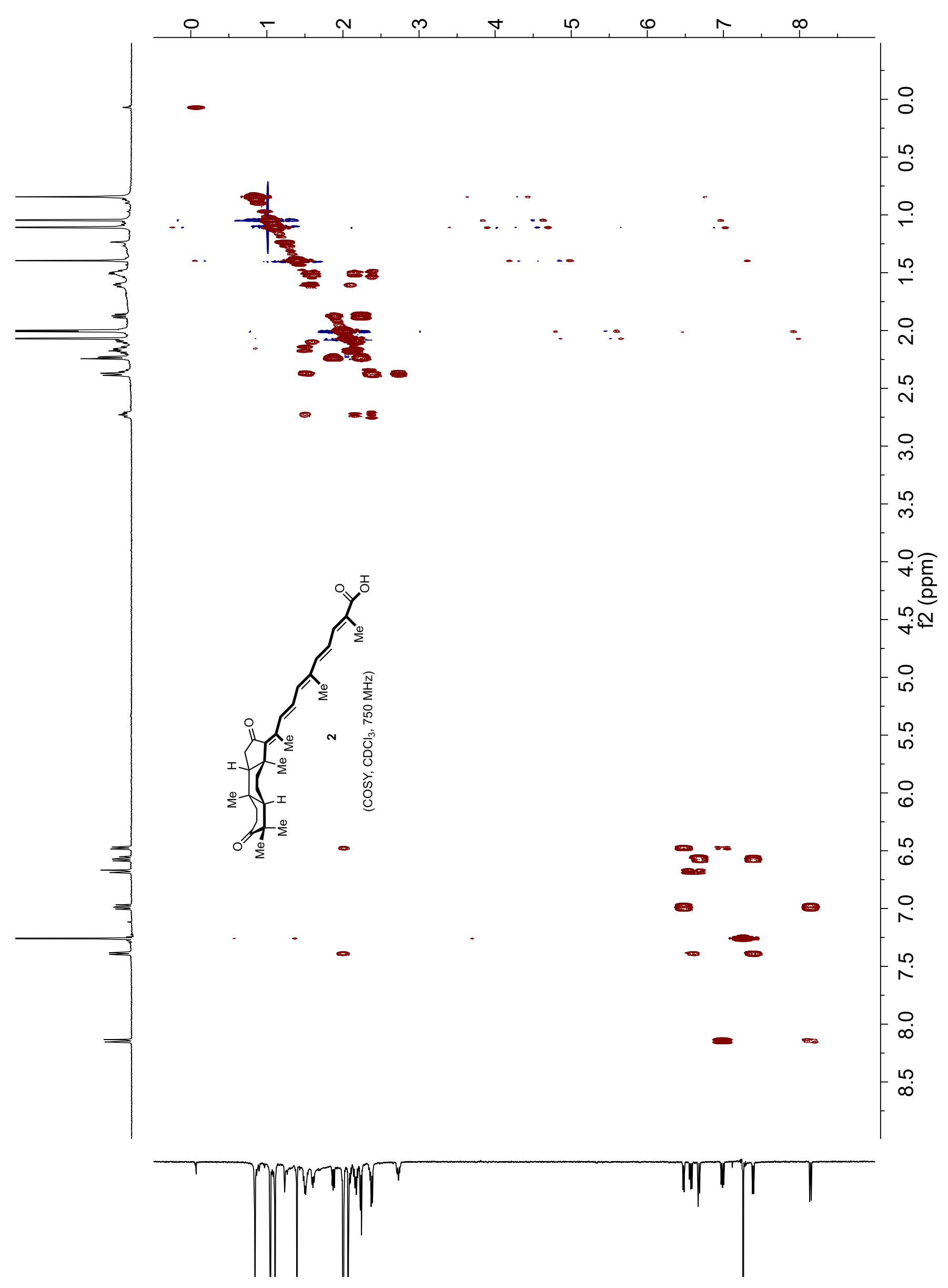


(mdd) If

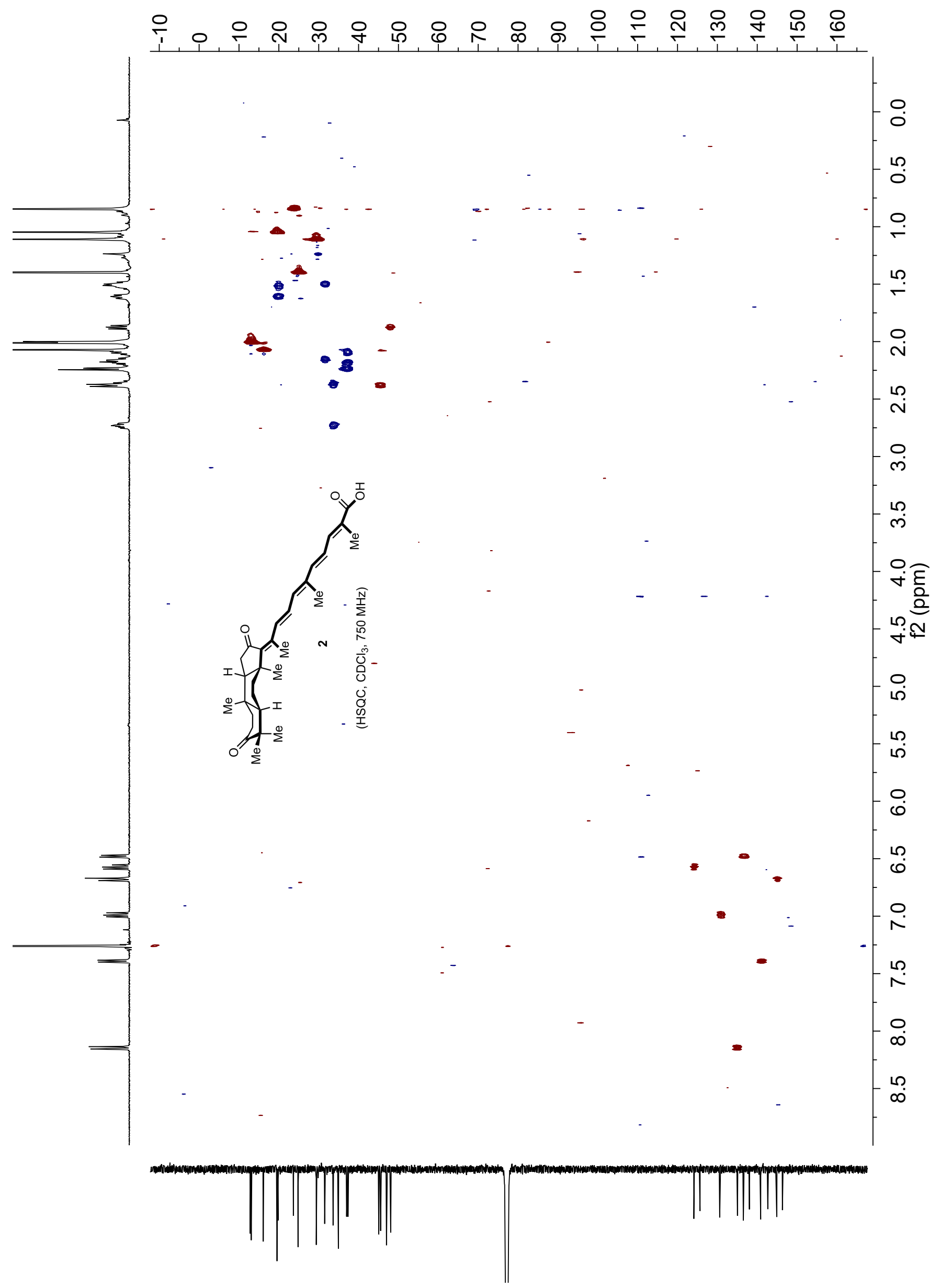


(mdd) If
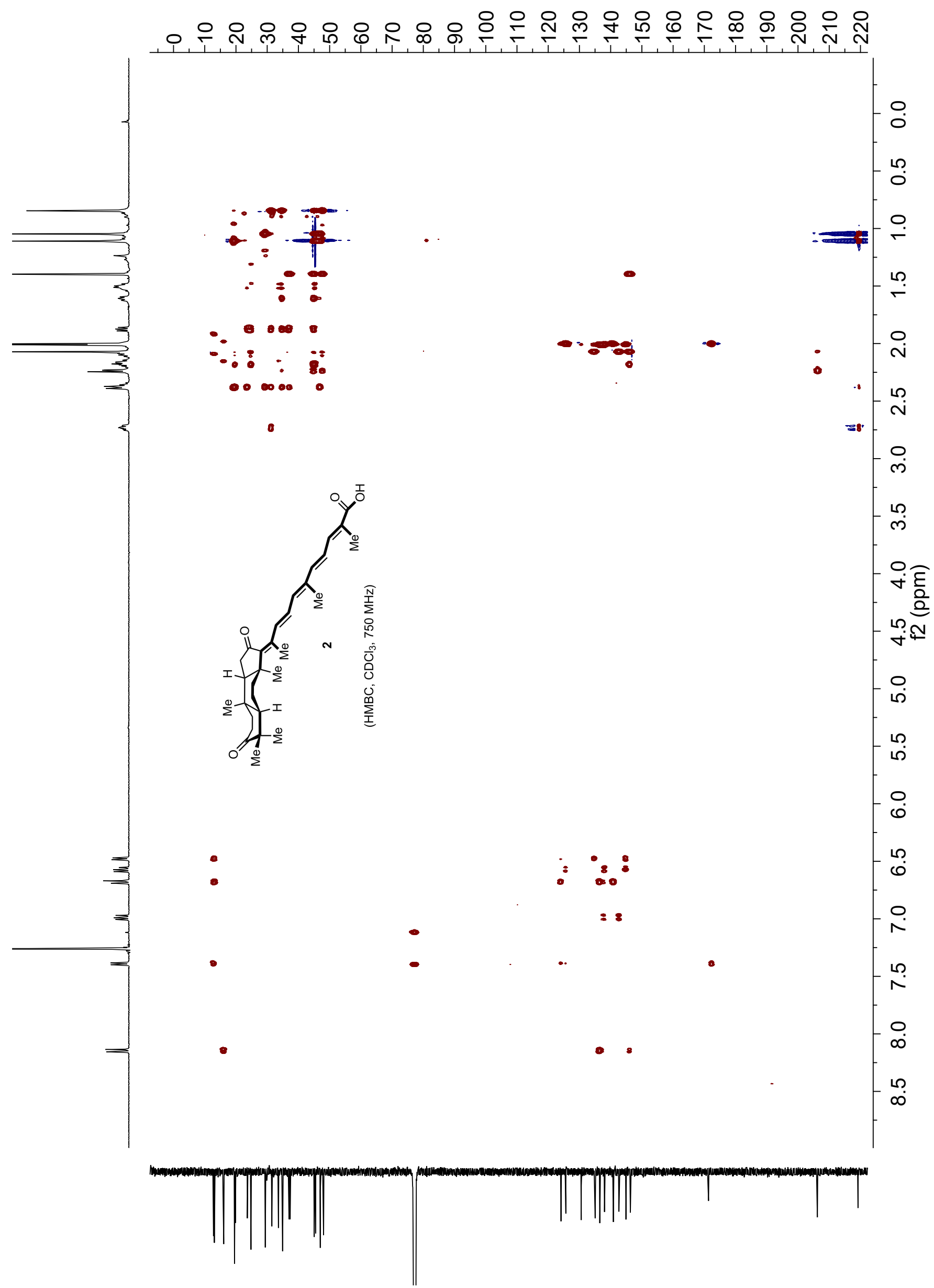